\title{
New $\boldsymbol{\beta}$-Lactam- $\boldsymbol{\beta}$-Lactamase Inhibitor Combinations
}

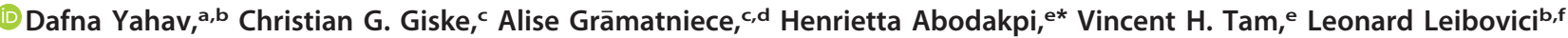 \\ alnfectious Diseases Unit, Rabin Medical Center, Beilinson Hospital, Petah-Tikva, Israel \\ bSackler Faculty of Medicine, Tel-Aviv University, Ramat Aviv, Israel \\ cClinical Microbiology, Department of Laboratory Medicine, Karolinska Institute and Karolinska University Hospital, Stockholm, Sweden \\ apauls Stradins University Hospital, University of Latvia, Riga, Latvia \\ eDepartment of Pharmacy Practice and Translational Research, University of Houston College of Pharmacy, Houston, Texas, USA \\ fMedicine E, Rabin Medical Center, Beilinson Hospital, Petah-Tikva, Israel
}

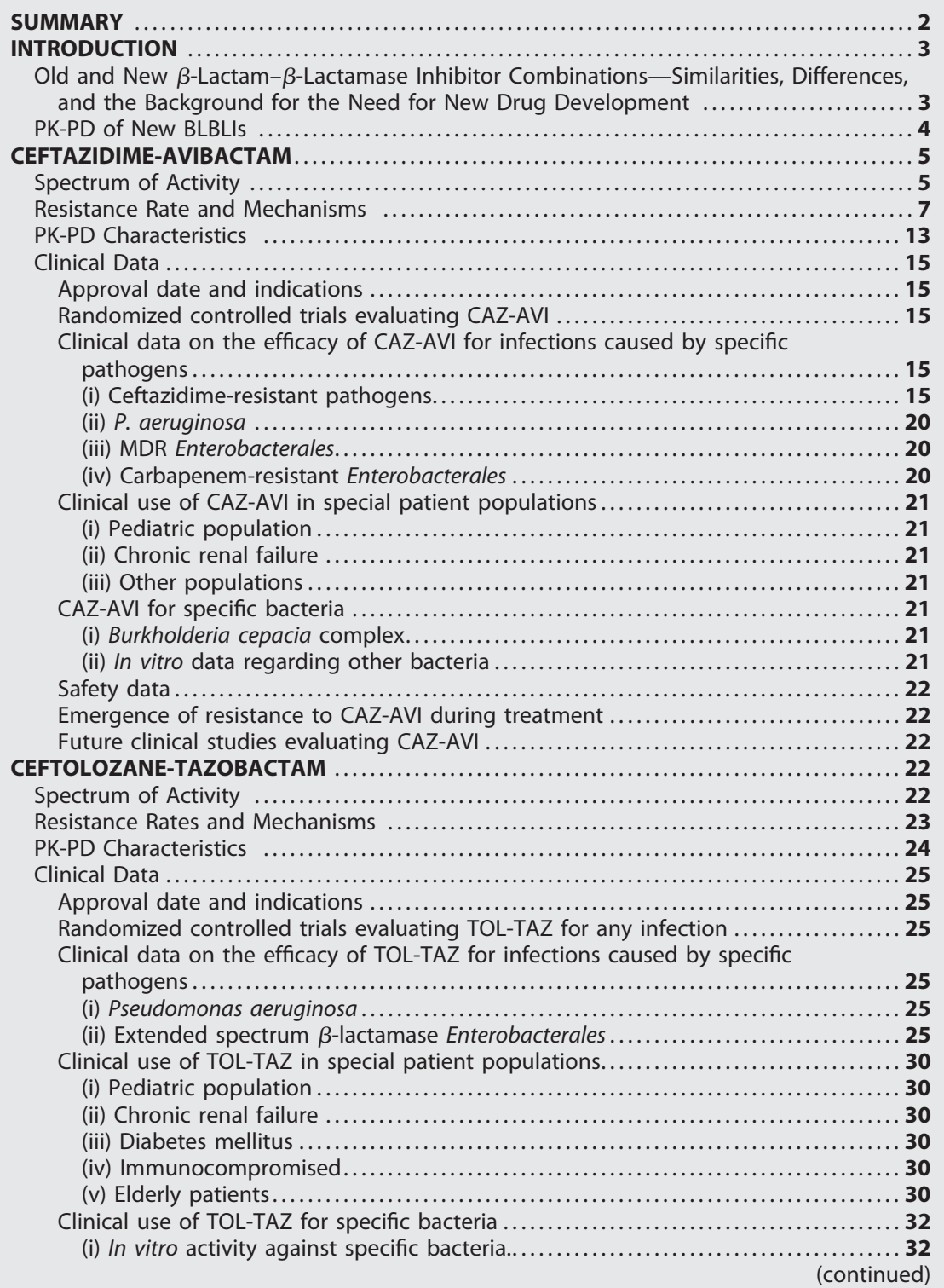

Citation Yahav D, Giske CG, Grāmatniece A, Abodakpi H, Tam VH, Leibovici L. 2021. New $\beta$-lactam- $\beta$-lactamase inhibitor combinations. Clin Microbiol Rev 34:e00115-20. https://doi .org/10.1128/CMR.00115-20

Copyright $\odot 2020$ American Society for Microbiology. All Rights Reserved.

Address correspondence to Dafna Yahav, dafna.yahav@gmail.com.

* Present address: Henrietta Abodakpi, United States Food and Drug Administration, Office of Clinical Pharmacology, Silver Spring, Maryland, USA

Published 11 November 2020 


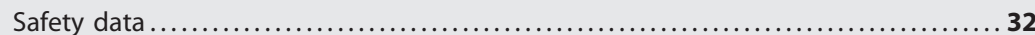

Emergence of resistance to TOL-TAZ during treatment............................ 32

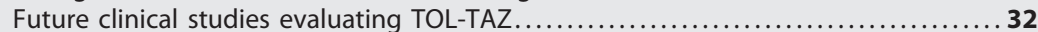

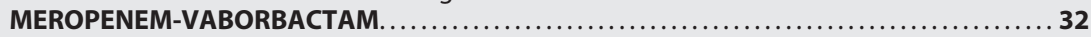

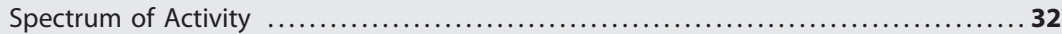

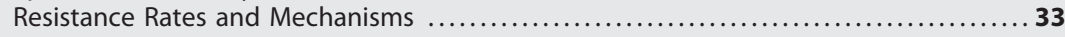

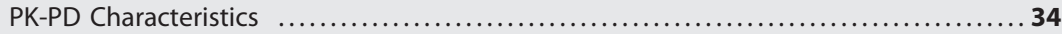

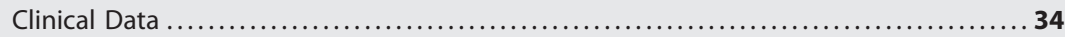

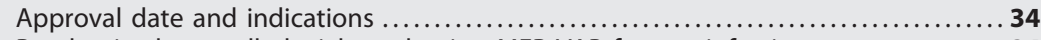

Randomized controlled trials evaluating MER-VAB for any infection............... 34

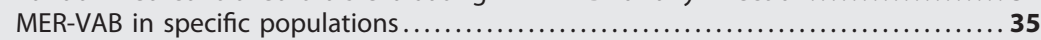

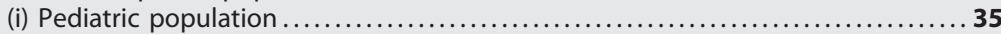

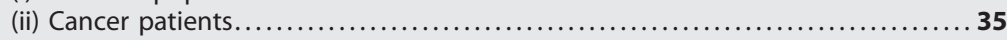

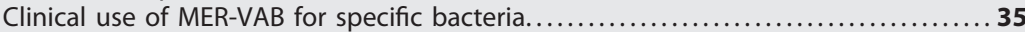

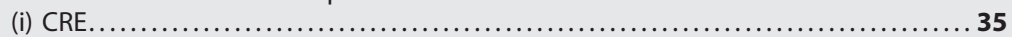

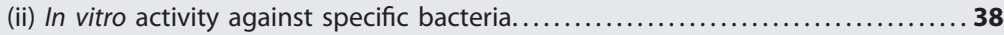

Emergence of resistance to MER-VAB during treatment .......................... 38

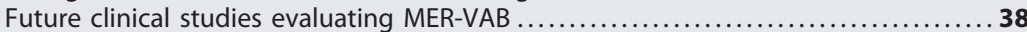

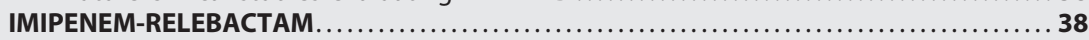

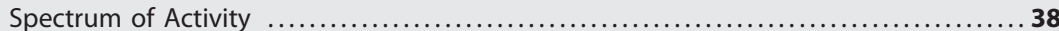

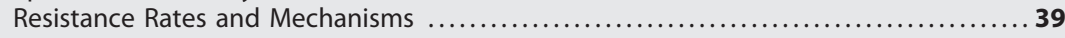

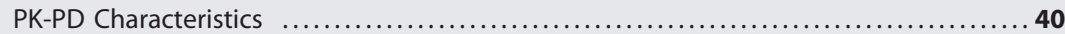

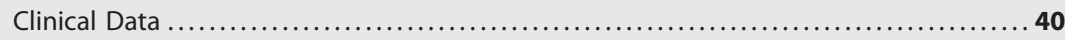

Approval date and indications ............................................ 40

Randomized controlled trials evaluating IMI-REL for any infection.............. 40

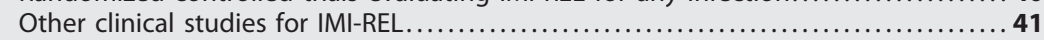

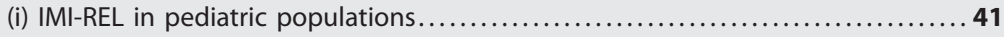

(ii) Other clinical studies.................................................. 41

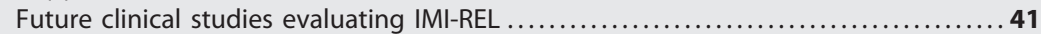

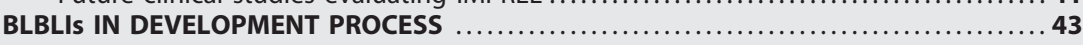

$\beta$-Lactam-Sulfone $\beta$-Lactamase Inhibitor Combinations $\ldots \ldots \ldots \ldots \ldots \ldots \ldots \ldots \ldots, 43$

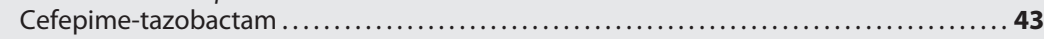

Cefepime-enmetazobactam (AAl101) ..................................... 43

$\beta$-Lactam-Diazabicyclooctane $\beta$-Lactamase Inhibitor Combinations ................ 45

Cefepime-zidebactam (WCK 5107) ...................................... 45

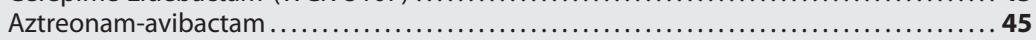

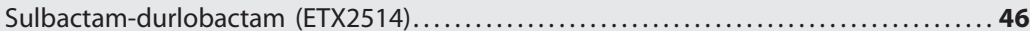

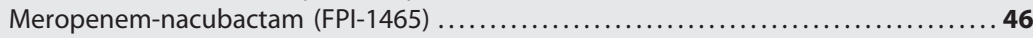

Cefpodoxime proxetil-ETX0282 (active compound ETX1317) ...................... 47

$\beta$-Lactam-Boronate $\beta$-Lactamase Inhibitor Combinations ..................... 47

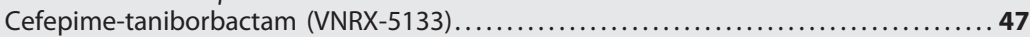

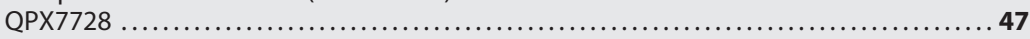

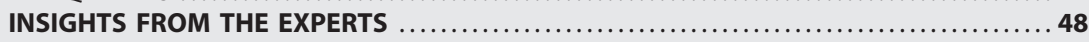

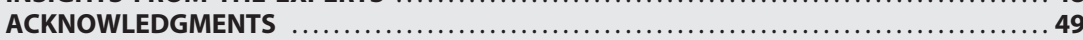

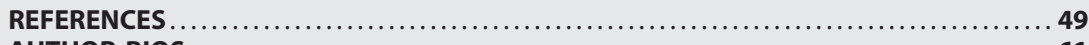

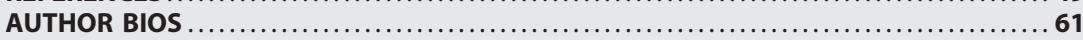

SUMMARY The limited armamentarium against drug-resistant Gram-negative bacilli has led to the development of several novel $\beta$-lactam- $\beta$-lactamase inhibitor combinations (BLBLIs). In this review, we summarize their spectrum of in vitro activities, mechanisms of resistance, and pharmacokinetic-pharmacodynamic (PK-PD) characteristics. A summary of available clinical data is provided per drug. Four approved BLBLIs are discussed in detail. All are options for treating multidrug-resistant (MDR) Enterobacterales and Pseudomonas aeruginosa. Ceftazidime-avibactam is a potential drug for treating Enterobacterales producing extended-spectrum $\beta$-lactamase (ESBL), Klebsiella pneumoniae carbapenemase (KPC), AmpC, and some class D $\beta$-lactamases (OXA-48) in addition to carbapenem-resistant Pseudomonas aeruginosa. Ceftolozane-tazobactam is a treatment option mainly for carbapenem-resistant $P$. aeruginosa (non-carbapenemase producing), with some activity against ESBL-producing Enterobacterales. Meropenem-vaborbactam has emerged as treatment option for Enterobacterales producing ESBL, KPC, or AmpC, with similar activity as meropenem against $P$. aeruginosa. Imipenem-relebactam has documented activity against Enterobacterales producing ESBL, KPC, and AmpC, with the combination having some additional activity against $P$. aeruginosa relative to imipenem. None of these drugs present in vitro activity against Enterobacterales or $P$. aeruginosa 
producing metallo- $\beta$-lactamase (MBL) or against carbapenemase-producing Acinetobacter baumannii. Clinical data regarding the use of these drugs to treat MDR bacteria are limited and rely mostly on nonrandomized studies. An overview on eight BLBLIs in development is also provided. These drugs provide various levels of in vitro coverage of carbapenem-resistant Enterobacterales, with several drugs presenting in vitro activity against MBLs (cefepime-zidebactam, aztreonam-avibactam, meropenem-nacubactam, and cefepime-taniborbactam). Among these drugs, some also present in vitro activity against carbapenem-resistant $P$. aeruginosa (cefepime-zidebactam and cefepimetaniborbactam) and A. baumannii (cefepime-zidebactam and sulbactam-durlobactam).

KEYWORDS $\beta$-lactam- $\beta$-lactamase inhibitor combinations, ceftazidime-avibactam, ceftolozane-tazobactam, meropenem-vaborbactam, imipenem-relebactam

\section{INTRODUCTION}

nfections caused by drug-resistant Gram-negative bacilli have become an important public health threat. Older drugs available to treat these infections, such as colistin, fosfomycin, aminoglycosides, and tigecycline, are limited in their efficacy, safety profile (e.g., colistin and nephrotoxicity), and by the emergence of resistance. The development of drugs active against these pathogens is a top priority. The World Health Organization (WHO) has issued a priority list of pathogens to direct efforts for drug development. Carbapenem-resistant Pseudomonas aeruginosa and cephalosporin/carbapenem-resistant Enterobacterales were listed as a critical priority (1). The Infectious Diseases Society of America (IDSA) launched in 2010 an initiative calling for development and approval of 10 new antibiotics effective against resistant Gram-negative bacteria by 2020 (2).

One of the prominent groups of new antibiotics with broad spectrum activity is the $\beta$-lactam- $\beta$-lactamase inhibitor combinations (BLBLIs). Several such combinations are currently in different stages of development and approval. In the manuscript, we aimed to review these drugs' features, including spectrum of activity, current resistance rates, pharmacokinetics/pharmacodynamics (PK-PD), clinical data on efficacy, adverse events, and what is known on their potential to select for resistance and cross-resistance. We aimed to put emphasis on clinical data regarding infections caused by resistant bacteria and subgroups of patients at high risk for such infections, such as immunocompromised patients. The main drugs of interest were those already approved for use, with a brief review on drugs still in development stages.

Old and New $\boldsymbol{\beta}$-Lactam- $\boldsymbol{\beta}$-Lactamase Inhibitor Combinations-Similarities, Differences, and the Background for the Need for New Drug Development

$\beta$-Lactams are a broad class of bactericidal agents that have been integral to the treatment of infections caused by Gram-positive and Gram-negative pathogens. The bactericidal activity of $\beta$-lactams is mediated by inhibition of penicillin-binding proteins (PBPs) essential to cell wall formation. The development of $\beta$-lactamase inhibitors has contributed to the preservation of the efficacy of $\beta$-lactams against $\beta$-lactamaseproducing pathogens. Traditionally, these inhibitors lack antibacterial activity at clinically relevant concentrations, and consequently, there are no model regulatory pathways for their development as stand-alone agents. Instead, they are coformulated with a partner $\beta$-lactam based on two key considerations: (i) the activity of the inhibitor against $\beta$-lactamases capable of hydrolyzing the $\beta$-lactam, and (ii) similarities in pharmacokinetic properties (such as elimination half-lives, metabolic pathways, and biodistribution) to ensure the protection of the $\beta$-lactam's structural integrity over a given dosing interval (3).

Optimal dosing of a BLBLI would likely be patient specific and is a complex interplay between the pathogen, bacterial burden at the site of infection, $\beta$-lactamase(s) involved, $\beta$-lactamase transcription level(s), the involvement of other resistance mechanism(s), the potency of the inhibitor, the potency of the $\beta$-lactam, the pharmacokinetics/biodistribution of the $\beta$-lactamase inhibitor, and the pharmacokinet- 
ics/biodistribution of the $\beta$-lactam. With the exception of amoxicillin-clavulanic acid, commercially available in different $\beta$-lactam-to-inhibitor ratios, all other commercially available BLBLIs are supplied as fixed dose ratio combinations. While the rationale for these fixed dose pairings is less apparent for older combinations such as piperacillintazobactam (PIP-TAZ), the development programs for newer combinations lend some insights into the selection of commercial dose ratio formulations. Dose ratios of BLBLIs are based (in part) on in vitro activity and in vivo antimicrobial efficacy. For instance, ceftolozane-tazobactam (TOL-TAZ) is featured as a 2:1 ratio of ceftolozane to tazobactam, because this ratio resulted in comparatively lower MICs against extendedspectrum $\beta$-lactamase (ESBL)-producing strains than either the $8: 1$ or 4:1 dose ratio (4). Additionally, the 2:1 ratio was found to yield either comparable or greater reductions in $\log _{10}$ CFU than other ratios evaluated in a murine thigh infection model.

Similarly, the commercial 4:1 ratio of ceftazidime-avibactam was supported by survival studies in infected mice treated with various ratios of ceftazidime-avibactam (5). While these commercial formulations have exhibited high rates of efficacy in clinical trials, allowing some flexibility in the pairing and dosing of the individual components of the combination may be warranted in some clinical scenarios (such as when enzyme hyperproduction or severe [high inoculum] infections are encountered). The design of stand-alone inhibitors could also facilitate expedient pairings to suit unique clinical needs (e.g., readily pairing aztreonam with avibactam against isolates suspected of harboring class $\mathrm{A}$ and $\mathrm{B} \beta$-lactamases).

The optimal approach for susceptibility testing for BLBLIs is a topic of ongoing debate. For susceptibility testing of older combinations (such as amoxicillinclavulanic acid and ampicillin-sulbactam), a fixed ratio of $\beta$-lactam to inhibitor is used, though concentrations vary. For newer combinations, in vitro susceptibility testing is conducted with a fixed concentration of inhibitor. For ampicillinsulbactam, the fixed 2:1 ratio employed in susceptibility testing reflects the 2:1 dose ratio used in all commercial formulations. Given the similarities in pharmacokinetics of ampicillin and sulbactam, this susceptibility testing arrangement is expected to reflect the concentration ratios achievable in vivo (6). For clavulanic acid, this same 2:1 ratio is used for in vitro susceptibility testing. Yet, the rationale for this practice is less evident, as amoxicillin-clavulanic acid is available in various fixed dose ratios as oral and intravenous (i.v.) formulations. While CLSI has always used a fixed ratio, the approach of EUCAST has been to use a fixed concentration for antibiotic susceptibility testing of combinations of aminopenicillins and inhibitors.

For combinations such as PIP-TAZ, ceftazidime-avibactam (CAZ-AVI), and imipenemrelebactam (IMI-REL), a fixed inhibitor concentration of $4 \mu \mathrm{g} / \mathrm{ml}$ is used alongside a range of parent $\beta$-lactam concentrations. While the concentrations of $\beta$-lactam reflect the dynamic concentrations observed in vivo, a static concentration of inhibitor is assumed to be adequate. In this scheme, it is generally expected that beyond the threshold concentration of $4 \mathrm{mg} /$ liter, the impact of the inhibitor concentration on MIC is minimal. However, if in vivo concentrations that range beyond this value are associated with further MIC reductions (i.e., nonsaturable effect), the fixed concentration approach may not reflect realistically the contribution of the inhibitor on MIC reduction and, ultimately, in vivo efficacy.

\section{PK-PD of New BLBLIs}

Traditionally, antibacterial drug development has relied on the identification of PK-PD indices (such as the Maximum concentration of the free, unbound drug in serum $\left[f C_{\text {max }}\right] / M I C$, area under the concentration-time curve for the free, unbound fraction of a drug $[\mathrm{fAUC}] / \mathrm{MIC}$, and cumulative percentage of a 24-h period that the free, unbound drug concentration exceeds the MIC under steady-state pharmacokinetic conditions $\left.\left[\% \mathrm{fT}_{>\mathrm{MIC}}\right]\right)$ through preclinical in vitro and in vivo dose fractionation studies to describe the killing activity of antibiotics. It is well established that the fraction of a 24-h period wherein free drug concentrations exceeds the MIC (i.e., \%fT $>$ MIC) is the PK-PD index predictive of microbiologic efficacy for $\beta$-lactams. The magnitude of $\% f T_{>\text {MIC }}$ required 
TABLE 1 Reported activity of various $\beta$-lactamase inhibitors from the BLBLIs against $\beta$ lactamase enzymes

\begin{tabular}{cllll}
\hline & Inhibited by: & & & \\
\cline { 2 - 5 } Enzyme & Avibactam & Tazobactam & Vaborbactam & Relebactam \\
\hline Class A & & No & Yes & Yes \\
KPC & Yes & Yes & Yes & Yes \\
SHV & Yes & Yes & Yes & Yes \\
TEM & Yes & Yes & Yes & Yes \\
CTX-M & Yes & & & No \\
Class B & & No & No & \\
MBL & No & & & Yes \\
Class C & & No & Yes & \\
AmpC & Yes & & & VD \\
Class D & & No & No & \\
OXA & VD & & &
\end{tabular}

${ }^{a} \mathrm{VD}$, variable data.

for maximum bactericidal effect derived from preclinical and clinical PK-PD studies ranges from $40 \%$ to $70 \%$ for the various subclasses of $\beta$-lactams (7). While these targets are often defined with respect to plasma concentrations, consideration of the extent of distribution of any antibiotic is critical to ensuring adequate exposure at the site of infection. Population PK modeling and Monte Carlo simulations have been used to predict the probability of achieving these PK-PD targets in patients, propose susceptibility breakpoints, and support proposed dosing regimens (8).

For $\beta$-lactamase inhibitors, however, delineating the driver of efficacy is a relatively novel and complex undertaking (9-13). Since currently approved $\beta$-lactamase inhibitors lack appreciable intrinsic antimicrobial activity, PK-PD studies are carefully designed to demonstrate the contribution of the inhibitor in the combination. The most widely used approach involves the identification of a PK-PD index to characterize the effect of the $\beta$-lactamase inhibitor and has been applied to inhibitors in all 4 of the most recently approved BLBLIs (see below) $(4,5,14,15)$. However, unlike $\beta$-lactams, there is no consensus PK-PD index used to describe the efficacy of all $\beta$-lactamase inhibitors (16).

Clinical data on optimal dosing (intermittent versus continuous infusion) and dosing in special patient populations (such as the critically ill) are generally lacking for newer BLBLIs. As hydrophilic drugs, $\beta$-lactams and $\beta$-lactamase inhibitors have a characteristically low volume of distribution $(V)$, akin to extracellular water, and are predominantly renally excreted (17). Thus, variations in extracellular volume and renal dysfunction often observed in critically ill patients may impact the disposition of both $\beta$-lactams and $\beta$-lactamase inhibitors (18). The current body of PK-PD knowledge surrounding the newly approved BLBLIs is discussed in the subsequent sections of this review.

\section{CEFTAZIDIME-AVIBACTAM}

\section{Spectrum of Activity}

Ceftazidime is hydrolyzed by class A ESBLs and carbapenemases, class B carbapenemases, and class $C$ cephalosporinases but not by most class $D$ carbapenemases. Avibactam inhibits class $A$, class $C$, and some of the class $D \beta$-lactamases, providing the combination a broad coverage of Gram-negative bacilli (Table 1) (19-24). Contrarily, the activity of CAZ-AVI against class B $\beta$-lactamase-producing isolates, Gram-negative anaerobes, and Gram-positive bacteria is limited $(25,26)$.

Published data have shown that CAZ-AVI is active against Enterobacterales, including ESBL-producing and AmpC-producing isolates, as well as some of the bacteria producing class D carbapenemases, such as OXA-24, OXA-40, OXA-69 (in Acinetobacter baumannii) and OXA-48 (in Klebsiella pneumoniae) (27-35). In the global surveillance study INFORM (International Network for Optimal Resistance Monitoring), 34,062 isolates of Enterobacteriaceae were collected between 2012 and 2014, and the overall suscepti- 


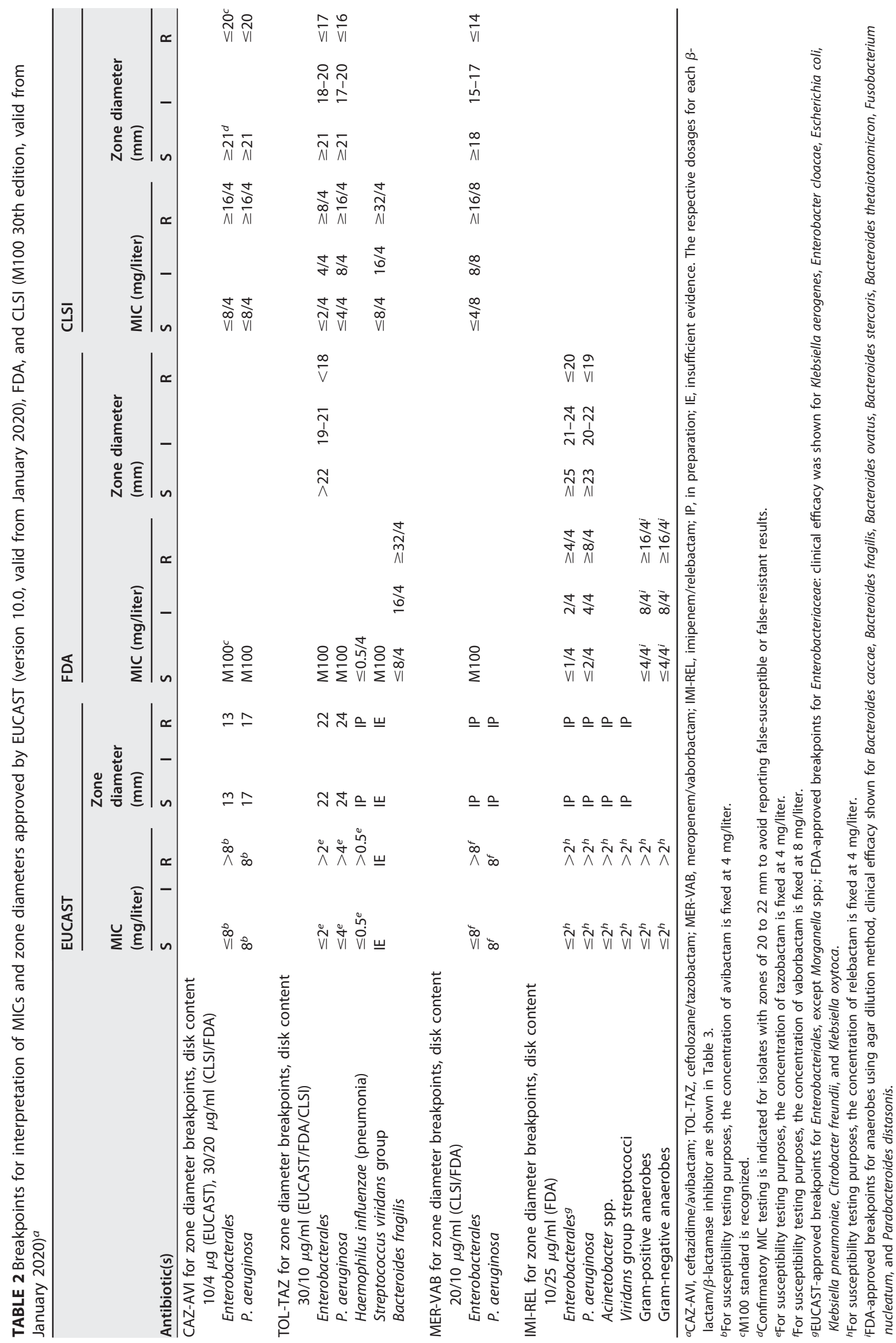


TABLE 3 Antimicrobial susceptibility of isolates to ceftazidime-avibactam

\begin{tabular}{lllll}
\hline & & \multicolumn{2}{l}{ MIC (mg/liter) } & \\
\cline { 3 - 4 } Pathogen $^{a}$ & No. of isolates & $\mathbf{5 0 \%}$ & $\mathbf{9 0 \%}$ & Susceptible (\%) \\
\hline Enterobacteriaceae & 3,269 & 0.12 & 0.5 & $99.9^{b}$ \\
Pseudomonas aeruginosa & 2,215 & 2 & 8 & $96.6^{b}$ \\
\hline
\end{tabular}

aUnited States isolates, 2019 (276).

${ }^{b}$ CLSI- and EUCAST-approved breakpoints applied.

bility to CAZ-AVI using Food and Drug Administration (FDA)-approved breakpoints was 99.5\%. The combination was active against ESBL- and AmpC-producing isolates of Escherichia coli, K. pneumoniae, Klebsiella oxytoca, and Proteus mirabilis (36). Subsequent data from the INFORM, including 16,656 Enterobacteriaceae isolates between 2015 and 2016 , showed that CAZ-AVI was active against $99.9 \%$ to $100.0 \%$ of the respective isolates (37). Meanwhile, in meropenem-nonsusceptible strains collected between 2015 and 2017, 73.0\% were susceptible to CAZ-AVI (38). The combination has also shown activity against bacteria carrying $b l a_{\mathrm{KPC}-2,} b l a_{\mathrm{KPC}-3}$, or bla $a_{\mathrm{OXA}-48 \text {-like }}$ genes $(39,40)$. A recent study showed that a combination of CAZ-AVI with aztreonam is active in resistant Enterobacter isolates carrying $b a_{\mathrm{NDM}-1}$ and $b a_{\mathrm{KPC}-4}$ on conjugative plasmids. The combination was demonstrated to show in vitro synergism. In addition, since aztreonam is not hydrolyzed by NDM, the addition of avibactam provides protection against class A enzymes to allow its action (41). The combination CAZ-AVI with aztreonam has also been described to successfully control a case of persistent bacteremia caused by Stenotrophomonas maltophilia carrying L1 (metallo- $\beta$-lactamase [MBL]) and L2 (cephalosporinase) $\beta$-lactamases (42). Analyzing resistant strains of $P$. aeruginosa, several studies have reported a high susceptibility rate to CAZ-AVI (43-47). However, in a study on piperacillin-tazobactam-resistant $P$. aeruginosa isolates from cystic fibrosis patients, the proportion with resistance to CAZ-AVI was $37.5 \%$. The resistant isolates showed inactivating mutations in the chromosomal porin encoding gene oprD (48). In a recently published study including $P$. aeruginosa isolates collected between 2013 and 2018, 35.9\% were resistant to CAZ-AVI, most of them identified as sequence type 235 (ST235) clone (49). The respective EUCAST-, FDA-, and CLSIapproved breakpoints for the interpretation of the susceptibility of particular pathogens to CAZ-AVI are summarized in Table 2. For antimicrobial susceptibility data of various pathogens to CAZ-AVI, see Tables 3 and 4 .

\section{Resistance Rate and Mechanisms}

The most common mechanism of resistance against CAZ-AVI is the presence of class $B$ and some of the class $D \beta$-lactamases (OXA-24/40 in A. baumannii, but not OXA-10 or OXA-48 in Enterobacterales) (50). Other mechanisms would include increased activity in the efflux pump, the loss of porins, and increased expression of the bla $a_{\mathrm{KPC}}$ gene $(51$, 52). Also, single point mutations in PBPs are associated with resistance to ceftazidime, which is not reversible with avibactam (53). Several studies have reported emerging CAZ-AVI resistance after the exposure, i.e., due to the mutations on the $\Omega$-loop of Klebsiella pneumoniae carbapenemase (KPC) enzymes leading to enhanced ceftazidime hydrolysis, which is not completely inhibited by avibactam (54-56). At Ambler amino acid position 179 in the $\Omega$-loop, substitutions of tyrosine for aspartic acid (D179Y) or asparagine for aspartic acid (D179N) have been reported to confer resistance to CAZ-AVI, combined with additional mutations outside the loop (57). Avibactam has been demonstrated to cause strong AmpC induction for some Enterobacter cloacae and $P$. aeruginosa strains, but no induction for Citrobacter freundii strains in one study (58). However, in another publication, no induction for E. cloacae was demonstrated (59). The clinical relevance of induction is not clear. An additional mechanism of resistance recently described is the acquisition of other $\beta$-lactamases, such as Vietnamese extended-spectrum $\beta$-lactamase (VEB)-25 (60).

Addressing $P$. aeruginosa isolates collected between 2005 and 2008. the overall resistance rate against CAZ-AVI was $18 \%$, mostly related to loss of porin and efflux 
TABLE 4 Antimicrobial susceptibility to BLBLIs

\begin{tabular}{|c|c|c|c|c|c|c|}
\hline Pathogen (no. of isolates, source [reference]) & Yr published & Sample collection yr(s) & \multicolumn{4}{|c|}{$\%$ of isolates susceptible to: ${ }^{a}$} \\
\hline \multicolumn{7}{|l|}{ Pseudomonas aeruginosa } \\
\hline$n=3,193$, U.S. (182) & 2020 & 2014-2018 & & & $88.8-89.5^{c}$ & \\
\hline$n=1,445$, Spain $(220)$ & 2020 & 2017 & $94.2^{b}$ & $94.6^{b}$ & & $97.3^{b}$ \\
\hline$n=414$, globally (278) & 2020 & & $93.0^{c}$ & $97.0^{c}$ & & \\
\hline$n=1,513$, U.S. (124) & 2019 & 2012-2016 & & $97.5^{b, c}$ & & \\
\hline$n=188$, Italy $(280)$ & 2019 & 2010-2016 & $85.1^{b}$ & & & \\
\hline$n=1,794$, Latin America (281) & 2019 & 2012-2015 & $87.4^{c}$ & & & \\
\hline$n=100$, Taiwan (282) & 2019 & 2016-2019 & $91.0^{c}$ & $93.0^{c}$ & & \\
\hline$n=2,215$, U.S. (276) & 2019 & 2017-2018 & $96.0^{b, c}$ & $95.9^{b, c}$ & & \\
\hline$n=433$, U.S. $(283)$ & 2019 & 2015-2017 & $98.2^{b, c}$ & $98.7^{b, c}$ & & \\
\hline$n=524$, China $(128)$ & 2019 & 2017 & $86.5^{c}$ & $88.5^{c}$ & & \\
\hline$n=355$, U.S. $(286)$ & 2018 & 2013-2015 & & $97.5^{c}$ & & \\
\hline$n=1,909$, U.S. $(287)$ & 2018 & 2017 & $96.9^{b, c}$ & $97.5^{b, c}$ & & \\
\hline$n=100$, Australia (288) & 2018 & 2008-2018 & & $96.0^{b, c}$ & & \\
\hline$n=368$, China $(289)$ & 2018 & 2012-2014 & $75.6^{c}$ & & & \\
\hline$n=1,259$, U.S. $(22)$ & 2018 & 2015-2017 & $96.8^{c}$ & & & \\
\hline$n=423$, U.S. $(22)$ & 2018 & 2017 & $96.2^{c}$ & $96.5^{c}$ & & \\
\hline$n=5,716$, Europe (290) & 2018 & 2012-2015 & $92.4^{b}$ & & & \\
\hline$n=56$, globally $(84)$ & 2018 & & $66.1^{c}$ & & & \\
\hline$n=4,175$, U.S. $(37)$ & 2018 & 2015-2016 & $97.6^{c}$ & & & \\
\hline$n=4,175$, U.S. $(291)$ & 2018 & 2011-2015 & $96.5^{c}$ & & & \\
\hline$n=935$, Italy $(292)$ & 2018 & 2013-2014 & & $90.9^{c}$ & & \\
\hline$n=3,737$, U.S. (293) & 2018 & 2013-2016 & & $97.3^{b, c}$ & & \\
\hline$n=489$, Asia-Pacific Region (125) & 2018 & 2013-2015 & & $90.8^{b, c}$ & & \\
\hline$n=210$, U.S. (299) & 2016 & 2012-2014 & $97.1^{c}$ & & & \\
\hline$n=1,257$, U.S. $(123)$ & 2015 & 2013-2014 & & $97.0^{c}$ & & \\
\hline$n=490$, U.S. (216) & 2015 & 2013-2014 & & & & $98.0^{c}$ \\
\hline$n=5,328$, U.S. $(300)$ & 2015 & $2011-2014$ & $96.8^{c}$ & & & \\
\hline Average susceptibility & & & $66.1-98.2$ & $86.8-99.8$ & $88.8-89.5$ & $90.8-100.00$ \\
\hline \multicolumn{7}{|l|}{ Pseudomonas aeruginosa, MDR } \\
\hline$n=3,770$, globally $(277)$ & 2020 & 2012-2016 & $68.2^{b}$ & & & \\
\hline$n=697$, U.S. (182) & 2020 & 2014-2018 & & & $59.0-59.7^{c}$ & \\
\hline$n=307$, U.S. (124) & 2019 & 2012-2016 & & $87.9^{b, c}$ & & \\
\hline$n=20$, Italy $(280)$ & 2019 & 2010-2016 & $70.0^{b}$ & & & \\
\hline$n=205$, Qatar (301) & 2019 & 2014-2015 & $68.8^{c}$ & $62.9^{c}$ & & \\
\hline$n=526$, U.S. (276) & 2019 & 2017-2018 & $83.5^{b, c}$ & $83.7^{b, c}$ & & \\
\hline$n=80$, Germany (49) & 2019 & 2013-2018 & $85.0^{b}$ & & & \\
\hline$n=750$, U.S. (302) & 2019 & 2015-2017 & & & & $79.7^{c}$ \\
\hline$n=3,708$, globally $(284)$ & 2019 & 2012-2016 & & & & $70.7^{c}$ \\
\hline$n=462$, Canada (126) & 2018 & 2008-2016 & & $90.5^{c}$ & & \\
\hline$n=327$, U.S. (22) & 2018 & 2015-2017 & $88.1^{c}$ & & & \\
\hline$n=121$, U.S. (22) & 2018 & 2017 & $86.2^{c}$ & $87.6^{c}$ & & \\
\hline$n=879$, U.S. (37) & 2018 & 2015-2016 & $89.3^{c}$ & & & \\
\hline$n=32$, U.S. (303) & 2018 & 2015 & $71.9^{c}$ & & & \\
\hline$n=783$, U.S. (293) & 2018 & 2013-2016 & & $88.6^{b, c}$ & & \\
\hline
\end{tabular}


TABLE 4 (Continued)

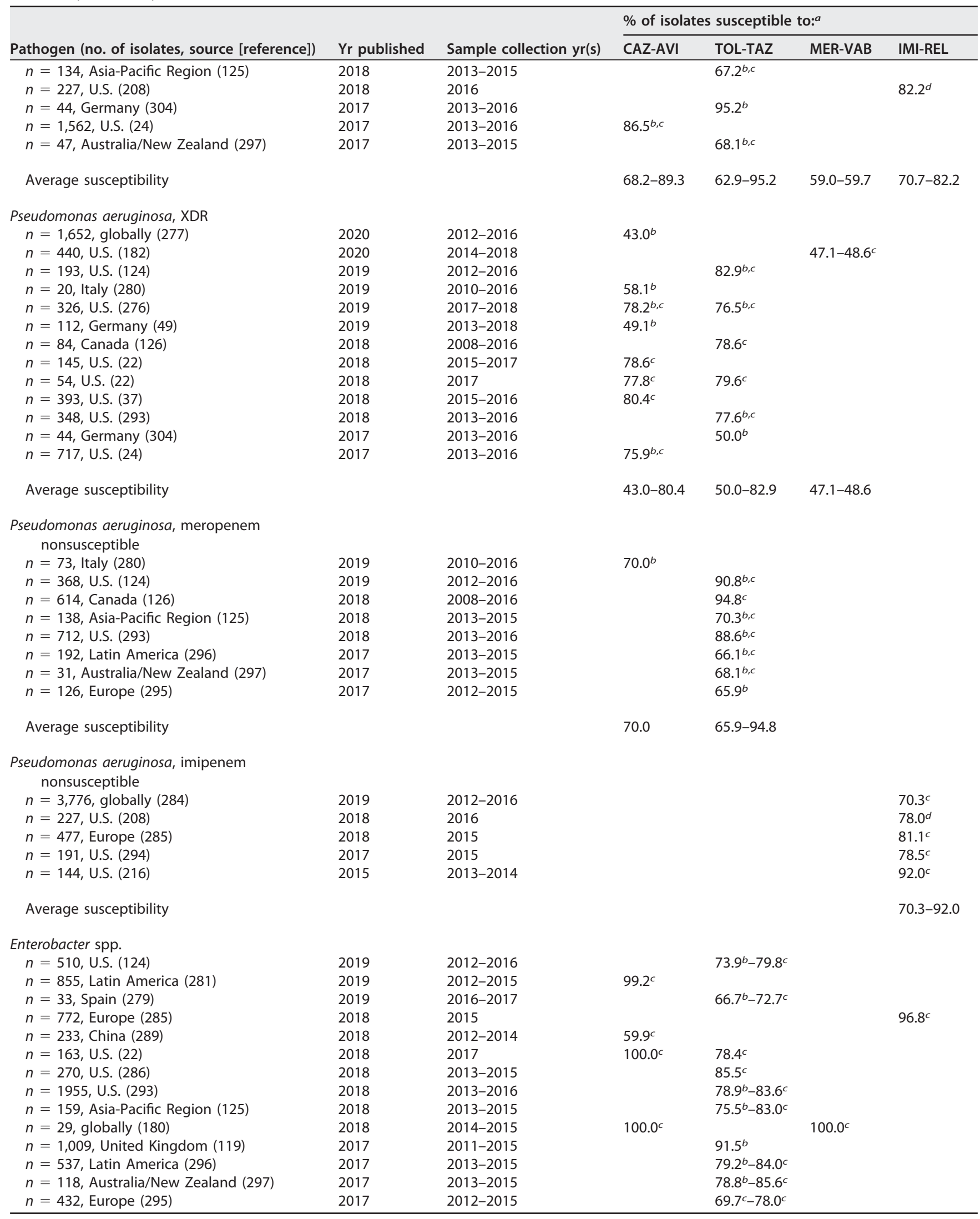


TABLE 4 (Continued)

\begin{tabular}{|c|c|c|c|c|c|c|}
\hline \multirow[b]{2}{*}{ Pathogen (no. of isolates, source [reference]) } & \multirow[b]{2}{*}{ Yr published } & \multirow[b]{2}{*}{ Sample collection yr(s) } & \multicolumn{4}{|c|}{$\%$ of isolates susceptible to: ${ }^{a}$} \\
\hline & & & CAZ-AVI & TOL-TAZ & MER-VAB & IMI-REL \\
\hline$n=211$, U.S. (216) & 2015 & $2013-2014$ & & & & $99.0^{c}$ \\
\hline$n=3970$, U.S. $(300)$ & 2015 & $2011-2014$ & $99.9^{c}$ & & & \\
\hline Average susceptibility & & & $59.9-100.0$ & $66.7-91.5$ & 100.0 & $96.8-96.8$ \\
\hline \multicolumn{7}{|l|}{ Enterobacter spp., imipenem nonsusceptible } \\
\hline \multicolumn{7}{|l|}{ Enterobacteriaceae } \\
\hline$n=273$, U.S. (124) & 2019 & 2012-2016 & & $87.5^{b}-90.3^{c}$ & & \\
\hline$n=1,347$, globally $(127)$ & 2019 & $2012-2016$ & & $89.4^{b}-91.4^{c}$ & & \\
\hline$n=7,665$, Latin America (281) & 2019 & 2012-2015 & $99.7^{c}$ & & & \\
\hline$n=3,269$, U.S. $(276)$ & 2019 & $2017-2018$ & $99.9^{b, c}$ & $86.9-90.1^{b, c}$ & & \\
\hline$n=3,746$, U.S. (283) & 2019 & $2015-2017$ & $99.9^{b, c}$ & $95.7-96.9^{b, c}$ & & \\
\hline$n=2,125$, U.S. $(22)$ & 2018 & $2015-2017$ & $100.0^{c}$ & & & \\
\hline$n=694$, U.S. (22) & 2018 & 2017 & $100.0^{c}$ & $92.0^{c}$ & & \\
\hline$n=509$, globally $(84)$ & 2018 & & $99.2^{c}$ & & & \\
\hline$n=18,656$, U.S. (37) & 2018 & $2015-2016$ & $99.9^{c}$ & & & \\
\hline$n=31,512$, U.S. (291) & 2018 & $2011-2015$ & $99.9^{c}$ & & & \\
\hline$n=15,223$, U.S. (293) & 2018 & $2013-2016$ & & $92.5^{b}-94.4^{c}$ & & \\
\hline$n=1,474$, Asia-Pacific Region (125) & 2018 & 2013-2015 & & $85.8^{b}-89.2^{c}$ & & \\
\hline$n=991$, globally $(180)$ & 2018 & 2014-2015 & $98.2^{c}$ & & $99.0^{c}$ & \\
\hline$n=3,419$, U.S. (208) & 2018 & 2016 & & & & $96.1^{d}$ \\
\hline$n=1,878$, Latin America (296) & 2017 & 2013-2015 & & $80.9^{b}-84.2^{c}$ & & \\
\hline$n=36,380$, U.S. (24) & 2017 & 2013-2016 & $99.9^{b, c}$ & & & \\
\hline$n=6,209$, U.S. $(30)$ & 2017 & $2011-2015$ & $99.9^{b, c}$ & & & \\
\hline$n=5,950$, Europe (295) & 2017 & 2012-2015 & & $91.3^{b}-93.5^{c}$ & & \\
\hline$n=391$, U.S. $(283)$ & 2019 & $2015-2017$ & $100.0^{b, c}$ & $83.2-88.8^{b, c}$ & & \\
\hline$n=285$, U.S. $(276)$ & 2019 & $2017-2018$ & $100.0^{b, c}$ & $76.8-84.1^{b, c}$ & & \\
\hline$n=271$, U.S. $(286)$ & 2018 & 2013-2015 & & $87.1^{b}$ & & \\
\hline$n=1,474$, Asia-Pacific Region (125) & 2018 & 2013-2015 & & $70.4^{b}-79.1^{c}$ & & \\
\hline$n=1,450$, U.S. (293) & 2018 & 2013-2016 & & $79.3^{b}-87.5^{c}$ & & \\
\hline$n=906$, Europe (295) & 2017 & 2012-2015 & & $74.9^{b}-82.8^{c}$ & & \\
\hline$n=67$, Australia/New Zealand (297) & 2017 & 2013-2015 & & $88.1^{b}-97.0^{c}$ & & \\
\hline$n=495$, Latin America (296) & 2017 & $2013-2015$ & & $66.9^{b}-74.7^{c}$ & & \\
\hline Average susceptibility & & & $99.9-100.0$ & $66.9-97.0$ & & \\
\hline \multicolumn{7}{|l|}{ Enterobacteriaceae, OXA-48 } \\
\hline$n=45,872$, globally $(247)$ & 2018 & 2012-2015 & $92.5^{c}$ & & & \\
\hline \multicolumn{7}{|l|}{ Carbapenem-resistant Enterobacteriaceae } \\
\hline$n=131$, U.S. $(182)$ & 2020 & 2014-2018 & & & $98.5-100.0^{c}$ & \\
\hline$n=216$, Italy $(306)$ & 2020 & 2016-2017 & $91.6^{b}$ & & & \\
\hline$n=97$, China $(307)$ & 2020 & & $78.4^{c}$ & & & \\
\hline$n=79$, U.S. (276) & 2019 & 2017-2018 & $97.5^{b, c}$ & $2.6^{b, c}$ & & \\
\hline$n=28$, U.S. $(283)$ & 2019 & 2015-2017 & $89.3^{b, c}$ & $0.0^{b, c}$ & & \\
\hline$n=372$, China $(128)$ & 2019 & 2017 & $75.3^{c}$ & $6.2^{c}$ & & \\
\hline$n=62$, U.S. (308) & 2019 & 2013-2016 & $87.1^{c}$ & $27.4^{c}$ & $79.0^{c}$ & $71.0^{c}$ \\
\hline$n=120$, U.S. (309) & 2019 & & $82.0^{c}$ & & $98.0^{c}$ & \\
\hline$n=330$, globally $(178)$ & 2018 & 2015 & & & $73.9^{c}$ & \\
\hline
\end{tabular}


TABLE 4 (Continued)

\begin{tabular}{|c|c|c|c|c|c|c|}
\hline Pathogen (no. of isolates, source [reference]) & Yr published & Sample collection yr(s) & \multicolumn{4}{|c|}{$\%$ of isolates susceptible to: ${ }^{a}$} \\
\hline$n=37$, U.S. (305) & 2018 & $2013-2015$ & & $2.7^{c}$ & & \\
\hline$n=238$, U.S. (37) & 2018 & 2015-2016 & $97.5^{c}$ & & & \\
\hline$n=286$, U.S. (293) & 2018 & 2013-2016 & & $2.4^{b}-4.5^{c}$ & & \\
\hline$n=513$, U.S. (24) & 2017 & 2013-2016 & $97.5^{b, c}$ & & & \\
\hline \multicolumn{7}{|l|}{ KPC-producing Enterobacteriaceae } \\
\hline$n=103$, U.S. $(218)$ & 2018 & & $100.0^{c}$ & & & $100.0^{c}$ \\
\hline \multicolumn{7}{|l|}{ Klebsiella spp. } \\
\hline$n=95$, Spain (279) & 2019 & 2016-2017 & & $66.3^{b}-72.9^{c}$ & & \\
\hline$n=692$, U.S. (286) & 2018 & 2013-2015 & & $93.1^{c}$ & & \\
\hline$n=246$, Australia/New Zealand (297) & 2017 & $2013-2015$ & & $96.3^{b}-98.8^{c}$ & & \\
\hline$n=1,484$, U.S. $(298)$ & 2016 & 2012-2014 & $99.9^{c}$ & & & \\
\hline Average susceptibility & & & $99.9-100.0$ & $66.3-98.8$ & & \\
\hline \multicolumn{7}{|l|}{ Klebsiella pneumoniae } \\
\hline$n=280$, globally $(127)$ & 2019 & 2012-2016 & & $80.0^{b}-83.6^{c}$ & & \\
\hline$n=2,128$, Latin America (281) & 2019 & 2012-2015 & $99.5^{c}$ & & & \\
\hline$n=100$, Taiwan (282) & 2019 & 2016-2019 & $100.0^{c}$ & $80.0^{c}$ & & \\
\hline$n=863$, U.S. $(276)$ & 2019 & $2017-2018$ & $100.0^{b, c}$ & $88.6-91.9^{b, c}$ & & \\
\hline$n=666$, China $(128)$ & 2019 & 2017 & $93.8^{c}$ & $52.7^{c}$ & & \\
\hline$n=1,591$, Europe $(285)$ & 2018 & 2015 & & & & $94.9^{c}$ \\
\hline$n=2,458$, globally $(178)$ & 2018 & 2015 & & & $97.0^{c}$ & \\
\hline$n=717$, U.S. (208) & 2018 & 2016 & & & & $99.4^{d}$ \\
\hline$n=238$, U.S. (294) & 2017 & 2015 & & & & $91.7^{c}$ \\
\hline$n=34$, globally $(225)$ & 2017 & 2012-2015 & & & & $100.0^{c}$ \\
\hline$n=594$, Latin America (296) & 2017 & 2013-2015 & & $60.4^{b}-64.6^{c}$ & & \\
\hline$n=917$, Europe $(295)$ & 2017 & 2012-2015 & & $75.8^{b-79.3^{c}}$ & & \\
\hline$n=190$, Australia/New Zealand (297) & 2017 & 2013-2015 & & $96.8^{b-98.4^{c}}$ & & \\
\hline$n=891$, U.S. $(216)$ & 2015 & 2013-2014 & & & & $99.3^{c}$ \\
\hline$n=1205$, U.S. $(123)$ & 2015 & 2013-2014 & & $89.0^{c}$ & & \\
\hline Average susceptibility & & & $74.2-100.0$ & $52.7-99.1$ & $97.0-98.9$ & $94.9-100.0$ \\
\hline \multicolumn{7}{|l|}{ Klebsiella pneumoniae, ESBL } \\
\hline$n=20$, Poland $(311)$ & 2019 & 2017 & & $65.0^{b}$ & & \\
\hline$n=22$, Spain $(279)$ & 2019 & $2016-2017$ & & $59.1^{b}-77.3^{c}$ & & \\
\hline$n=843$, globally $(178)$ & 2018 & 2015 & & & $91.2^{c}$ & \\
\hline$n=49$, U.S. (286) & 2018 & 2013-2015 & & $73.5^{c}$ & & \\
\hline$n=1,474$, Asia-Pacific Region (125) & 2018 & 2013-2015 & & $56.6^{b}-69.4^{c}$ & & \\
\hline$n=40$, Germany $(310)$ & 2018 & 2014-2015 & & $62.5^{b}$ & & \\
\hline$n=119$, U.K. (119) & 2017 & $2011-2015$ & & $84.0^{b}$ & & \\
\hline$n=226$, Latin America (296) & 2017 & $2013-2015$ & & $46.0^{b}-56.6^{c}$ & & \\
\hline$n=12$, Australia/New Zealand (297) & 2017 & 2013-2015 & & $66.7^{b}-83.3^{c}$ & & \\
\hline$n=373$, Europe $(295)$ & 2017 & $2012-2015$ & & $41.6^{b}-49.1^{c}$ & & \\
\hline
\end{tabular}


TABLE 4 (Continued)

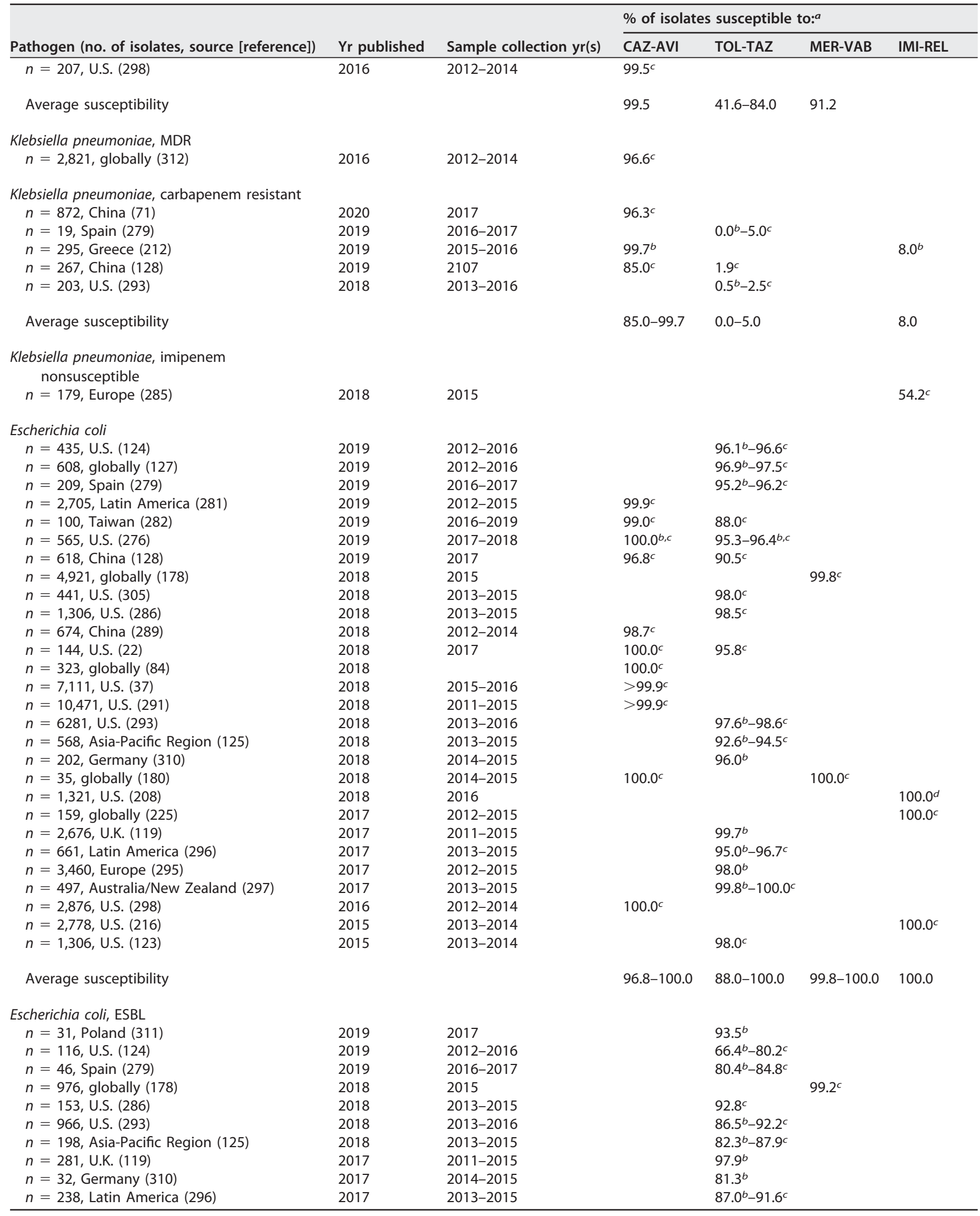


TABLE 4 (Continued)

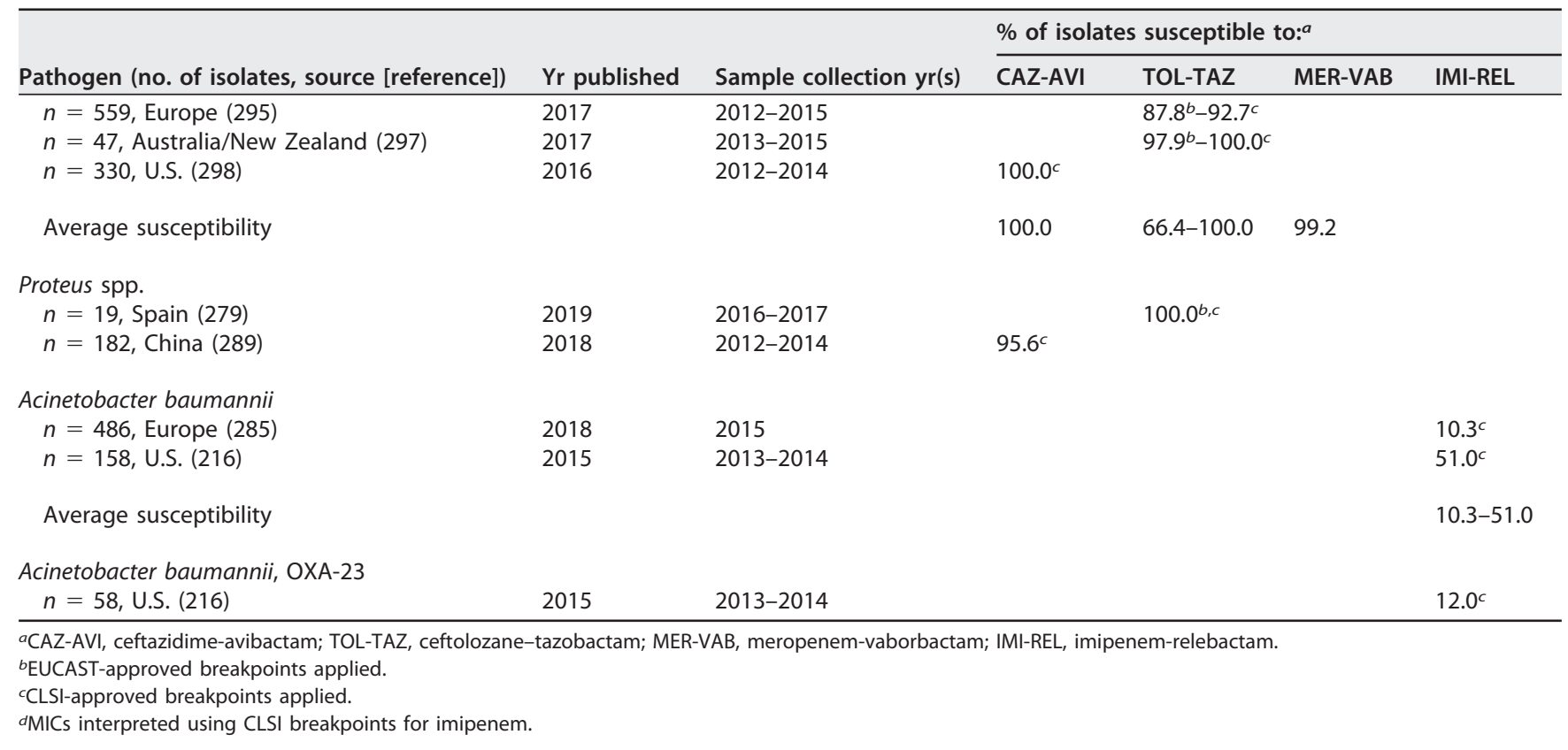

pump (61). Resistance due to the presence of VIM metallo- $\beta$-lactamase (MBL) or mutations in the chromosomal AmpC gene in $P$. aeruginosa isolates has also been reported (62-64). In Acinetobacter spp., resistance to CAZ-AVI is mainly due to a failure of avibactam to penetrate the outer membrane $(65,66)$.

In Citrobacter freundii, resistance to the combination has been documented due to mutations within the coding region of the bla $a_{\mathrm{KPC}-2} \Omega$-loop. (67) In cases of the CAZ-AVI-resistant $K$. pneumoniae, most of the isolates were ST258 mutant $b / a_{\mathrm{KPC}-3}(34$, $52,68,69)$. There are also reports of decreased CAZ-AVI susceptibility in KPC-producing K. pneumoniae isolates enhanced by OmpK35 porin deficiency $(53,70)$. A recent study from China reported $3.7 \%$ resistance rate to the CAZ-AVI in carbapenem-resistant $K$. pneumoniae, mainly due to the production of $\mathrm{MBL}$ but also in isolates harboring mutated $b / a_{\mathrm{KPC}-2}(\mathrm{D} 179 \mathrm{Y})$. In these isolates, avibactam does not inhibit the $\beta$-lactamase, enabling it to hydrolyze ceftazidime $(71,72)$. Cases of nonfunctional OmpK35-OmpK37 and altered OmpK36 porins associated with a higher copy number of the $b / a_{\mathrm{KPC}}$ gene have also been reported $(71,73)$.

Assessing the resistance development following exposure to CAZ-AVI, a study with KPC-producing $K$. pneumoniae reported that $8 \%$ of the isolates became resistant within 10 to 19 days of exposure (74). Similar data were reported by Gaibani et al. when the resistance in KPC-producing K. pneumoniae against CAZ-AVI emerged after 17 days of combination therapy with CAZ-AVI and gentamicin due to D179Y substitution in the $b / a_{\mathrm{KPC}-3}$ gene (56). Recently published findings in the K. pneumoniae ST307 documented the resistance development within 12 days of CAZ-AVI exposure through a $b / a_{\mathrm{KPC}-2}$ point mutation. Researchers also identified an additional phenotype with combined CAZ-AVI and meropenem resistance (55). Fraile-Ribot et al. (75) reported a case of carbapenem-resistant $P$. aeruginosa developing resistance to CAZ-AVI under the treatment. The resistant isolate contained a 3-bp insertion leading to the duplication of a key residue, designated OXA-539 (75). Moreover, the expeditious development of CTX-M-14 isoforms with increased ceftazidime hydrolytic activity may limit the usefulness of CAZ-AVI in monotherapy, in particular, against isolates carrying bla $a_{\mathrm{CTX}-\mathrm{M}-14}$ and $b / a_{\mathrm{OXA}-48}(54)$.

\section{PK-PD Characteristics}

Ceftazidime and avibactam exhibit numerous similarities in pharmacokinetic prop- 
TABLE 5 Pharmacokinetic properties of newly approved BLBLIs

\begin{tabular}{|c|c|c|c|c|c|c|c|c|}
\hline \multirow{2}{*}{ Parameter } & \multicolumn{8}{|c|}{ Value for drug combination by dose ratio ${ }^{a}$} \\
\hline & \multicolumn{2}{|c|}{ 4:1 (2.5 g q8h [over $2 \mathrm{~h}]):$} & \multicolumn{2}{|c|}{$\begin{array}{l}\text { 2:1 }(1.5 \mathrm{~g} \text { or } 3 \mathrm{~g} \text { q8h } \\
\text { [over } 1 \mathrm{~h}]):\end{array}$} & \multicolumn{2}{|c|}{$\begin{array}{l}\text { 1:1 (4 g q8h [over } \\
3 \mathrm{~h} \text { ]): }\end{array}$} & \multicolumn{2}{|c|}{$\begin{array}{l}\text { 2:1 }(1.25 \mathrm{~g} \mathrm{q} 6 \mathrm{~h} \\
\text { [over } 0.5 \mathrm{~h}]): b\end{array}$} \\
\hline$V_{\text {ss }}(\text { liter })^{c}$ & 17 & 22.2 & 13.5 & 18.2 & 20.2 & 18.6 & 24.3 & 19.0 \\
\hline Half-life (h) & 2.76 & 2.71 & 3.12 & 1.03 & 2.3 & 2.3 & 1.0 & 1.2 \\
\hline$\%$ protein bound & $<10$ & $5.7-8.2$ & $16-21$ & 30 & 2 & 33 & 20 & 22 \\
\hline \multicolumn{9}{|l|}{$\mathrm{CL}(\mathrm{ml} / \mathrm{min})^{e}$} \\
\hline Renal & 100 & 158 & $57-112$ & 210 & 130 & 99 & 115 & 123 \\
\hline Total & 115 & 218 & $68-112$ & 340 & 175 & 133 & 223 & 133 \\
\hline
\end{tabular}

aCAZ, ceftazidime; AVI, avibactam; TOL, ceftolozane; TAZ, tazobactam; MER, meropenem; VAB, vaborbactam; IMI, imipenem; REL, relebactam.

bFormulated as $500 \mathrm{mg}$ imipenem plus $500 \mathrm{mg}$ cilastatin plus $250 \mathrm{mg}$ relebactam.

${ }^{c} V_{s s}$, volume of distribution at steady state.

${ }^{d} \mathrm{AUC}$, area under the curve; ELF, epithelial lining fluid.

${ }^{e} \mathrm{CL}$, clearance.

erties: both have short plasma half-lives, low plasma protein binding, and similar volumes of distribution (Vs) and epithelial lining fluid (ELF) penetration ratios, as summarized in Table 5 . Additionally, both ceftazidime and avibactam are primarily renally excreted as unchanged drugs (76).

The clinical pharmacology program used to support the approval of CAZ-AVI by the FDA and European Medicines Agency (EMA) relied on the published plasma $\% f T_{>\text {MIC }}$ of ceftazidime required for efficacy against Enterobacterales and $P$. aeruginosa. For avibactam, it was proposed that adequate protection of ceftazidime (against $\beta$-lactamases) would allow the PK-PD of the combination to resemble that of ceftazidime alone. On that basis, the PK-PD index for avibactam was defined as a critical avibactam concentration below which sufficient inhibition of ceftazidime was lost (i.e., \%fT $>C_{T}$ ). (5) $A$ plasma target of $50 \%$ fT of $>1 \mu \mathrm{g} / \mathrm{ml}$ was determined through a series of dose fractionation studies with avibactam against a fixed backdrop of sub-MICs of ceftazidime in a neutropenic murine thigh infection model (5). In phase 2 studies with adult complicated intra-abdominal infection ( $\mathrm{ClAl}$ ) patients with normal renal function, $2 \mathrm{~g}$ ceftazidime was administered with $0.5 \mathrm{~g}$ avibactam as a 30-min i.v. infusion every $8 \mathrm{~h}$. However, population PK models predicted that joint probability of target attainment (which simultaneously evaluates the probability of achieving the respective PK-PD targets of ceftazidime and avibactam) would fall below the conventional threshold of $>90 \%$ used to support susceptibility breakpoints.

Since the efficacies of both ceftazidime and avibactam are described as time dependent, an extended duration of infusion ( $2 \mathrm{~h}$ ) was proposed to optimize dosing of the combination for phase 3 studies in complicated urinary tract infection (cUTI) and clAl patients. (5) For the treatment of hospital-acquired bacterial pneumonia (HABP) (including ventilator-associated bacterial pneumonia [VABP]), dose selection accounted for the disposition of ceftazidime and avibactam at the infection site by evaluating the ELF penetration ratio in a murine lung infection model and in healthy human volunteers. Ultimately, a $2.5 \mathrm{~g}$ dose ( $2 \mathrm{~g}$ ceftazidime plus $0.5 \mathrm{~g}$ avibactam) infused over $2 \mathrm{~h}$ was approved for all indications in patients with normal renal function.

Given that ceftazidime and avibactam are eliminated by the kidneys, various population PK models have illustrated creatinine clearance $(\mathrm{CrCL})$ to be a key covariate accounting for differences in the pharmacokinetics of both agents (5). Thus, dosage adjustment is recommended for patients with $\mathrm{CrCL}$ of $\leq 50 \mathrm{ml} / \mathrm{min}$, with specific recommendations for the different renal impairment groups (i.e., moderate versus severe versus end-stage renal diseases [ESRDs], estimated using the Cockcroft-Gault formula) based on achievement of $>90 \%$ probability of target attainment (PTA) while mitigating potential safety risks. Population PK models indicate that no dosage adjustments are required in the elderly or on the basis of race, body weight, or sex. In 
pediatric patients aged 3 months to 17 years with CIAI and CUTI, the approved dosage regimens of $50 \mathrm{mg} / \mathrm{kg}$ body weight to $2.5 \mathrm{~g} \mathrm{CAZ-AVI}$ (in patients with normal renal function) are designed to match the exposures corresponding to the approved adult dose, since the disease processes are age independent (76).

A recent retrospective study evaluating the use of CAZ-AVI for the treatment of infections due to carbapenem-resistant Enterobacterales (CRE) found pneumonia and the need for renal replacement therapy (RRT) as risk factors for clinical and microbiological failure (77). The higher rates of treatment failure in patients receiving RRT may be attributed to the absence of appropriate dosing recommendations for this patient cohort. However, the rationale for the higher rate of treatment failures in patients with pneumonia is less apparent. Nonetheless, these observations may point to a need to further evaluate the robustness of the fixed dose ratio approach for dosing CAZ-AVI irrespective of infection severity/site, causative pathogen, $\beta$-lactamase(s) present, or patient-specific factors.

\section{Clinical Data}

Approval date and indications. CAZ-AVI is approved by both the EMA and the FDA. It was first approved by FDA in 2015 for use in adults with cIAI (in combination with metronidazole) and CUTI, including pyelonephritis. It was later approved for use in pediatric patients 3 months and older for these indications and also for HABP/VABP in adults (76). EMA approval also refers to the indication of infections due to aerobic Gram-negative organisms in patients with limited treatment options (78).

Randomized controlled trials evaluating CAZ-AVI. Seven publications, representing 8 randomized controlled trials (RCTs) were published evaluating CAZ-AVI with or without metronidazole versus carbapenems/quinolones. For efficacy outcomes from these trials, see Table 6 . The representation of third-generation cephalosporin resistant Gram-negatives in these trials varied, between $11 \%(79)$ to $78 \%(21)$. Data from these RCTs evaluating the efficacy and safety of CAZ-AVI in adults were compiled in several meta-analyses. Sternbach et al. (80) reported results of eight trials, 4,093 patients, which compared CAZ-AVI with or without metronidazole versus any other antibiotic regimen (mostly carbapenem) for treatment of CUTI, ClAl, and nosocomial pneumonia. No difference in all-cause mortality at late follow-up was demonstrated in this metaanalysis between CAZ-AVI and the comparator, though the mortality in total was $\sim 3 \%$, probably limiting the external validity of these trials. The trials excluded immunocompromised patients, patients with severe renal or liver impairment, and patients not expected to respond to antibiotics within 5 to 21 days. No significant differences in clinical cure or microbiological cure at test of cure (TOC) were demonstrated as well. In the subgroup of patients with UTI, higher microbiological cure rates were demonstrated with CAZ-AVI at TOC. Similar results of higher microbiological cure with CAZ-AVI were also reported in a meta-analysis by Zhang et al., compiling trials of patients with CUTI and CIAI (81). Though no significant difference was demonstrated for the outcome of any adverse events (AEs), the rate of serious AEs (SAEs) was significantly higher with CAZ-AVI versus comparator, mostly carbapenem. Detailed data on the nature of these AEs were missing (80). Two additional meta-analyses did not demonstrate a significant difference between CAZ-AVI versus comparator in any efficacy or safety outcomes for infections caused by Enterobacterales (82) and serious Gram-negative infections (83). In the latter meta-analysis, a subgroup analysis of patients with CRE infections (4 studies, $n=281$ ) showed significantly lower mortality and higher clinical cure rates with CAZ-AVI. However, this subgroup analysis was based on three cohort studies and one post hoc analysis of a randomized controlled trial, limiting the validity of the results.

Clinical data on the efficacy of CAZ-AVI for infections caused by specific pathogens. (For details on studies addressing CAZ-AVI for resistant bacteria, see Table 7.)

(i) Ceftazidime-resistant pathogens. Overall, from available RCTs in adults, clinical response was reported for 782 patients with infection caused by ceftazidime-resistant pathogens, either Enterobacterales or P. aeruginosa. For these patients, clinical response 


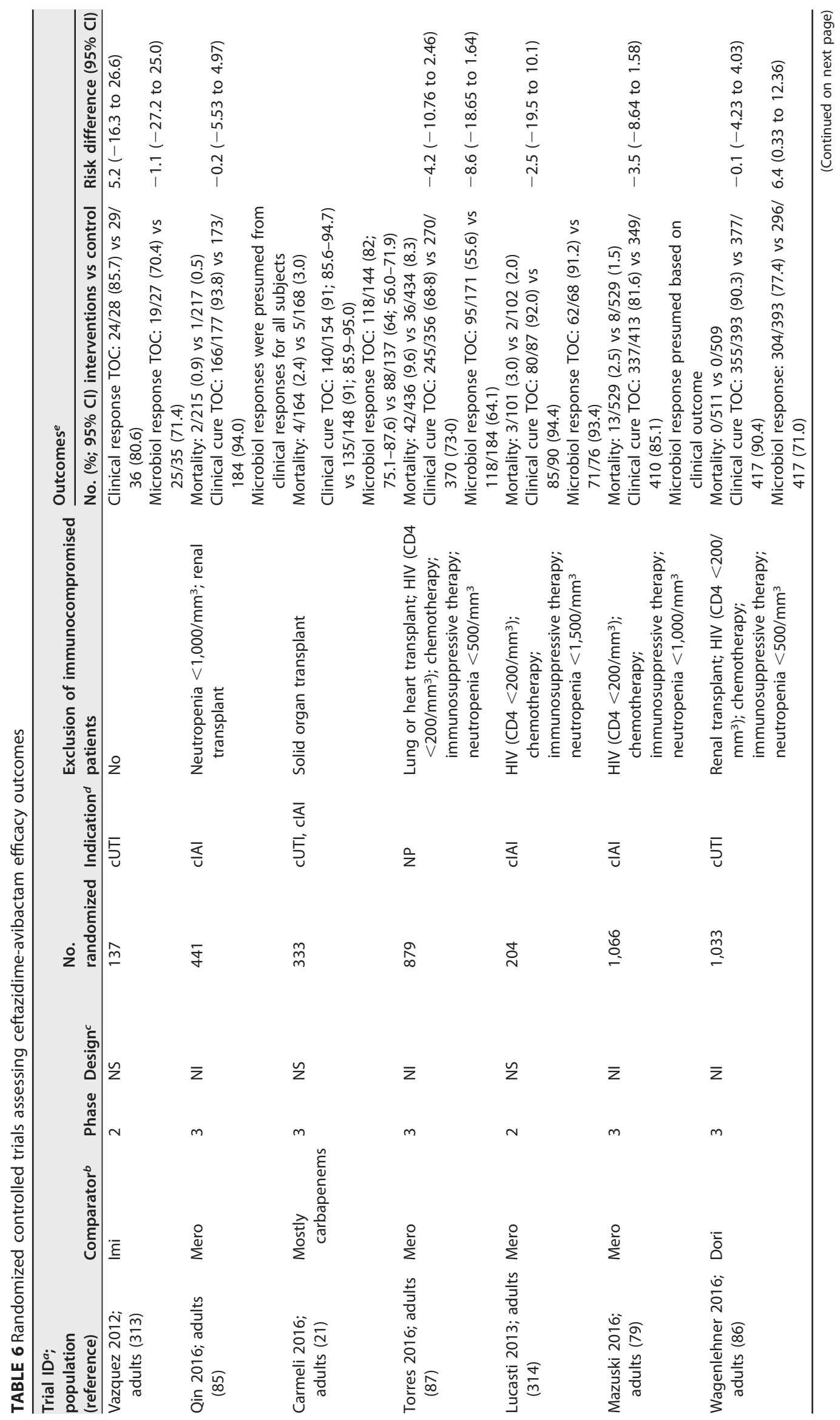




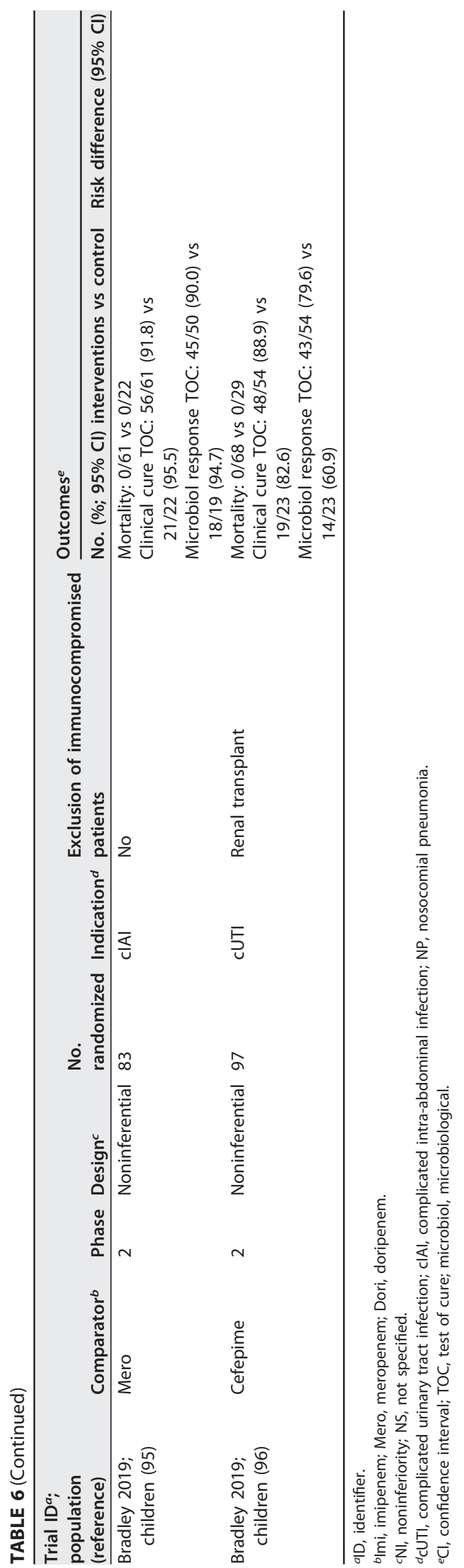




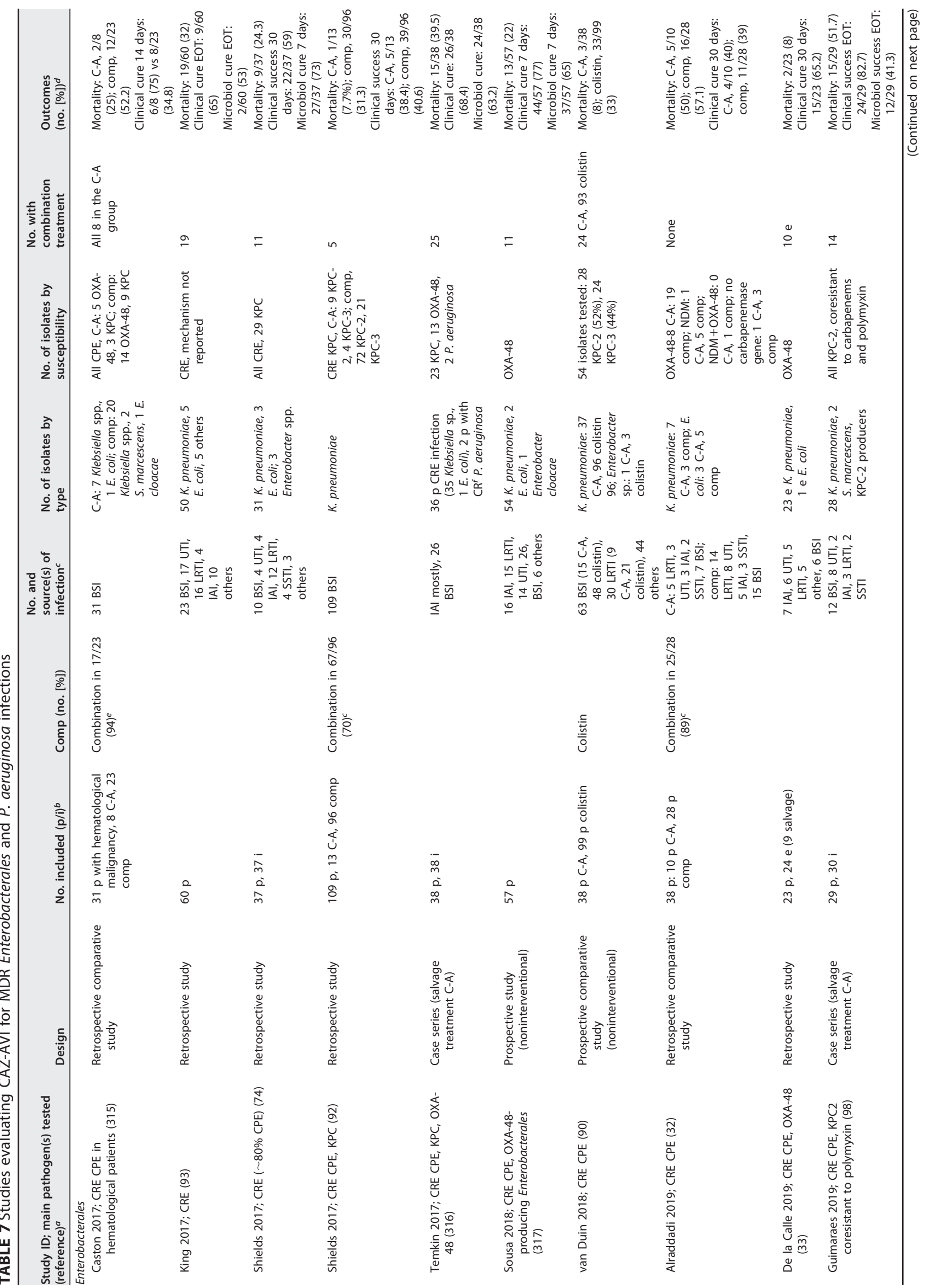




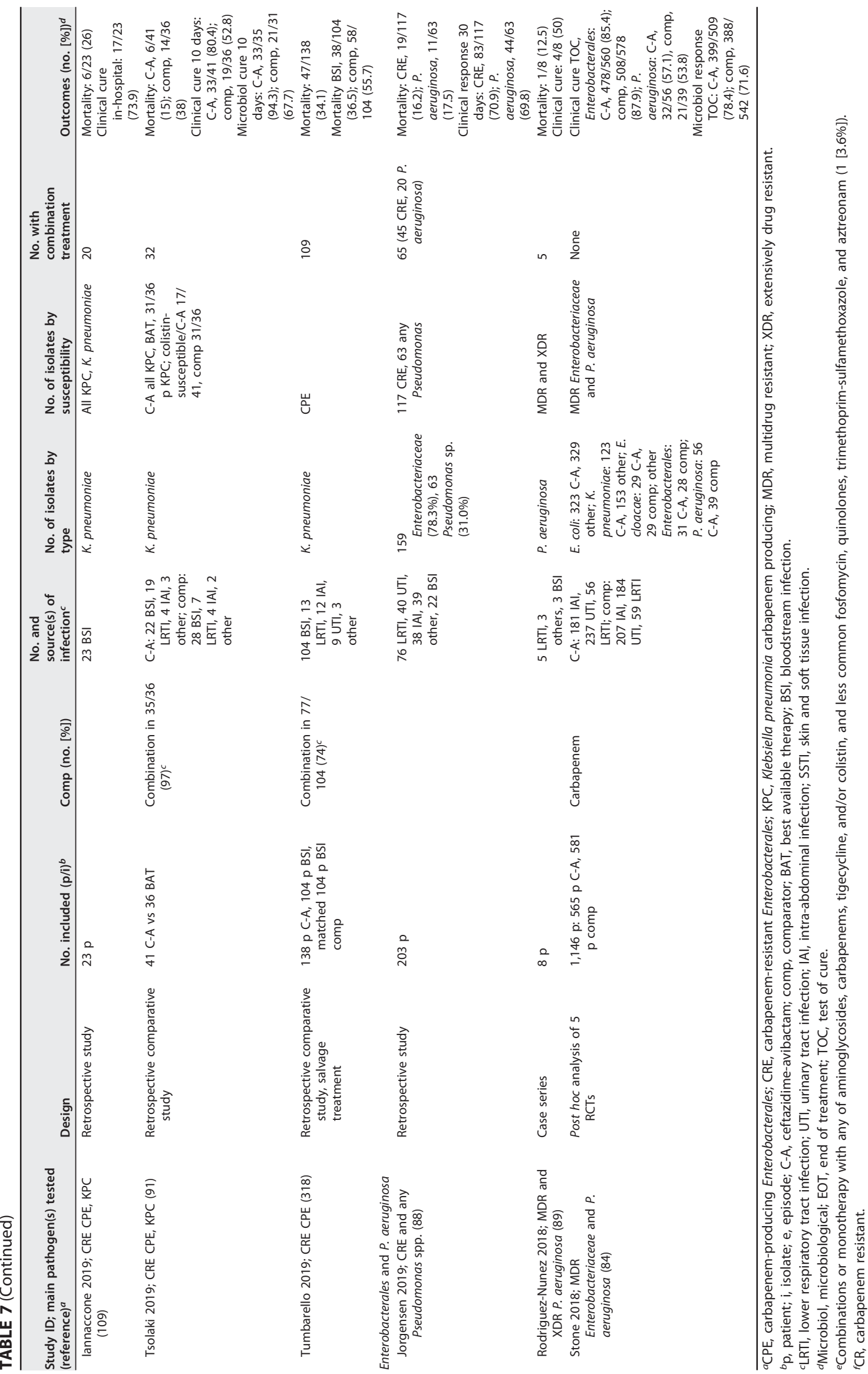


was $86 \%(326 / 378)$ in the CAZ-AVI arm versus $85 \%(342 / 404)$ in the comparator arm (carbapenem based) (80).

(ii) $P$. aeruginosa. Stone et al. (84) conducted a post hoc analysis of five randomized controlled trials $(21,79,85-87)$, evaluating outcomes among 1,146 patients with multidrug-resistant (MDR) pathogens treated with CAZ-AVI versus comparator; among them, 95 were MDR $P$. aeruginosa (see Table 7 for details). Mortality was not reported, but clinical cures reported specifically for $P$. aeruginosa were similar between study arms $(57.1 \%$ with CAZ-AVI versus $53.8 \%$ with the comparator). Microbiological responses for $P$. aeruginosa were similar between CAZ-AVI and comparator (32/56 [57.1\%] versus 21/39 [53.8\%], respectively, $P=0.83$ ). A few additional studies addressed CAZ-AVI effectiveness in $P$. aeruginosa infections: Jorgensen et al. (88) reported on 63 patients with Pseudomonas spp. infections, mostly pneumonia (60\%), treated with CAZ-AVI. Among the isolates tested, $47.5 \%$ were ceftazidime resistant and $77.5 \%$ were meropenem resistant. Mortality at 30 days was $11 / 63(17.5 \%)$, and clinical response rates were $69.8 \%$ (44/63) (88). One small case series reported on eight patients with MDR/extensively drug resistant (XDR) P. aeruginosa infections treated CAZ-AVI, with $50 \%$ clinical response (4/8) and $12.5 \%$ 30-day mortality (1/8) (89).

(iii) MDR Enterobacterales. Stone et al. (84), in their post hoc analysis, included 1,051 MDR Enterobacterales isolates. Clinical cures at test of cure were similar between CAZ-AVI and comparator for both Enterobacterales and $P$. aeruginosa (overall 478/560 [85.4\%] versus 508/578 [87.9\%], $P=0.222$ ). Microbiological response at TOC was significantly higher with CAZ-AVI for MDR Enterobacterales (399/509 [78.4\%] versus 388/542 [71.6\%], $P=0.013$ ) (84).

(iv) Carbapenem-resistant Enterobacterales. As mentioned above, one metaanalysis showed significantly lower mortality and higher clinical cure rates with CAZ-AVI versus those for the comparator in CRE infections (83). Specifically, for KPC, three comparative studies demonstrated significant reduction in mortality compared to that with the best available therapy (BAT) (90-92).

In addition, 14 nonrandomized studies evaluated CAZ-AVI for the treatment of CRE. These are summarized in Table 7. Five studies were comparative; most studies evaluated patients with CRE carbapenemase-producing Enterobacterales (CPE) isolates, but two studies did not report mechanism of carbapenem resistance $(88,93)$. Overall, 632 patients were treated with CAZ-AVI in these studies, demonstrating overall mortality (30-days or in-hospital mortality) of $25.6 \%$ (162/632) and a clinical response rate of $67.5 \%$ (318/471). Microbiological response at end of treatment (EOT) was 64.5\% (165/ 256) with CAZ-AVI. In one comparative study, higher microbiological response rate was demonstrated with CAZ-AVI than with BAT (mostly colistin/tigecycline based combination) (33/35 [94.3\%] versus 21/31 [67.7\%], respectively) (91).

Since these studies were mostly retrospective, using various comparators and various rates of combination therapy, and not all adjusting for a possible bias of receiving a new expensive drug versus the less expensive alternative, it is difficult to draw conclusions on the drug's efficacy.

Onorato et al. (94) performed a meta-analysis compiling 11 retrospective studies and case series, evaluating the efficacy of CAZ-AVI administered as monotherapy versus a combination for the treatment of CRE and carbapenem-resistant $P$. aeruginosa infections. Three hundred ninety-six subjects were included (only 19 with $P$. aeruginosa): 202 received the combination and 194 received monotherapy. The mortality rates were similar between combination therapy (38.1\%) and monotherapy (30.9\%). Similarly, no difference was found between the two groups for the outcome of microbiological cure. Clinical cure was not compiled in this meta-analysis. CAZ-AVI resistance emergence was reported in 6 patients $(3.0 \%)$ in the combination group and 8 patients $(4.1 \%)$ in the monotherapy group (94).

In summary, clinical data regarding the use of CAZ-AVI for CRE infections are limited to observational, mostly retrospective, noncomparative studies. Indications for using CAZ-AVI over older drugs in these studies were the failure of older drugs, risk factors for toxicity with older drugs or actual toxicity, or routine use in more recent periods, when 
the new drugs were readily available. Thus, no methodologically adequate comparison has ever been performed. Data regarding $P$. aeruginosa are scarce.

Clinical use of CAZ-AVI in special patient populations. (i) Pediatric population. Two phase II RCTs were conducted in a pediatric population (total, 180 patients), evaluating CAZ-AVI versus cefepime for CUTI and CAZ-AVI plus metronidazole versus meropenem for CIAI. No cases of fatality were reported in either of these trials. Clinical cure rates at TOC were similar between groups; microbiological cure rates were similar for CIAI and nonsignificantly higher with CAZ-AVI for CUTI (Table 7). Rates of any AEs were similar, and SAEs were nonsignificantly more common with CAZ-AVI (10.1\% versus $6.0 \%)(95,96)$.

losifidis et al. (97) reported a case series of 8 critically ill children less than 5 years old who received CAZ-AVI as part of combination therapy for XDR/pandrug-resistant (PDR) K. pneumoniae infections. At 30 days, all children survived the infection, and a favorable clinical and microbiological outcome was reported. In all children, no severe adverse events were reported, with no discontinuation or dose modification of the drug (97).

(ii) Chronic renal failure. Patients with severe renal or liver impairment were excluded from RCTs evaluating CAZ-AVI. Hence, data can be obtained only from observational studies, mostly evaluating CAZ-AVI for CRE infections, as detailed above. Shields et al. (74) reported lower clinical success among patients requiring continuous renal replacement therapy (CRRT) (1/6 [17\%] versus 21/31 [68\%]), though numbers are small. Among patients other than those requiring CRRT, success rates were not influenced by lower baseline creatinine clearance $(\mathrm{CrCL})$ (74). King et al. (93) evaluated CAZ-AVI for CRE infections in hematological patients. In this study, 33 patients required renal dose adjustment of the drug. Among them, mortality was $42 \%$ (14/33), clinical success was $58 \%$ (19/33), and microbiological response was 55\% (18/33), all similar to the entire cohort (93). De La Calle et al. reported 90-day mortality of $20 \%(2 / 10)$ and $60 \%(6 / 10)$ clinical cure among 10 patients with chronic renal failure at baseline (33). Guimaraes et al. (98) reported 44\% (4/9) mortality among nine patients who received adjusted renal dosage of CAZ-AVI, while 5 patients with renal impairment at baseline and no dose adjustment remained alive. These few data may suggest a need to reevaluate dose adjustment for renal impairment (98).

(iii) Other populations. (a) Immunocompromised patients. Most studies conducted in CRE patients (Table 7) included immunocompromised patients, such as organ transplant recipients ( $13 \%$ to $50 \%$ of patients included) and patients with malignancy ( $14 \%$ to $100 \%$ of included patients). Very few data are available for the outcomes specifically in these populations.

(b) Elderly patients. Clinical cures among older adults (aged 65 years and older) were similar between CAZ-AVI and comparator in 3 RCTs for 576 patients $(79,85,86)$.

CAZ-AVI for specific bacteria. (i) Burkholderia cepacia complex. Several case reports described the favorable clinical response to a CAZ-AVI-containing regimen in the treatment of infections caused by $B$. cepacia complex. These include one case report in a 2-month-old infant with B. cepacia bacteremia (99) and several cases of lung transplant and cystic fibrosis patients with B. cepacia serious infections (100-104). In an in vitro study, CAZ-AVI was demonstrated to have greater activity specifically against Burkholderia multivorans than ceftazidime $\left(\mathrm{MIC}_{90}\right.$ values of 4 and $16 \mathrm{mg} / \mathrm{liter}$, respectively). In this study, XDR Burkholderia strains were susceptible to CAZ-AVI in $22 \%$ of cases (inhibited by $\leq 4 \mathrm{mg} /$ liter of the drug). CAZ-AVI was less active against Burkholderia gladioli than against B. cepacia. (105).

(ii) In vitro data regarding other bacteria. (a) Mycobacteria. In an in vitro model, CAZ-AVI was demonstrated to be effective against Mycobacterium avium at concentrations achievable by using clinical doses. The MIC of ceftazidime in the presence of avibactam was $16 \mathrm{mg} /$ liter with the broth microdilution method (106). These findings were supported by an in vitro study showing favorable kill rates with a CAZ-AVI-based regimen. The drug achieved high intracellular penetration with intracellular concentrations above the MIC throughout the dosing interval (107). In addition, potential in vitro activity was also demonstrated for Mycobacterium tuberculosis. In a study using drug- 
resistant M. tuberculosis isolates, the MICs of $96 \%$ of 25 isolates were below CAZ-AVI peak concentrations, achieved at standard doses (108).

(b) Others. A recent study tested the in vitro activity of several BLBLIs against Burkholderia, Achromobacter, and Stenotrophomonas strains, most of them isolated from respiratory specimens collected from cystic fibrosis patients. CAZ-AVI was demonstrated to have activity $\left(\mathrm{MIC}_{50}\right.$ of $8 \mathrm{mg} /$ liter; $\mathrm{MIC}_{90}$ of $32 \mathrm{mg} /$ liter) against Achromobacter spp., with $78 \%$ of isolates susceptible to the drug according to CLSI breakpoints for $P$. aeruginosa; in vitro activity was also demonstrated against S. maltophilia, with $40 \%$ of isolates susceptible according to same breakpoints (105).

Safety data. As mentioned above, no significant difference in rates of any adverse events in general was demonstrated between CAZ-AVI and comparators in RCTs. Specifically, gastrointestinal AEs ( $\sim 20 \%$ of patients) and increase in creatinine ( $2 \%)$ were significantly more common with CAZ-AVI than with carbapenems. Other AEs reported included pyrexia, peripheral edema, hypersensitivity reactions, and neurological AEs, each in $\sim 3 \%$ to $6 \%$ of patients receiving CAZ-AVI. Sternbach et al. described higher rates of SAEs with CAZ-AVI in a meta-analysis of RCTs (80). Unfortunately, these SAEs are not described in detail in the trials. In nonrandomized studies, AEs were not comprehensively reported, and overall acute kidney injury (AKI) was reported in up to $5 \%$ of patients; other reported AEs were mainly gastrointestinal and neurological AEs, with sporadic cases of rash, leukopenia, and abnormal liver function.

Emergence of resistance to CAZ-AVI during treatment. Two RCTs reported $\geq 4$-fold increase in the MIC to CAZ-AVI during treatment in persistent Enterobacterales and $P$. aeruginosa strains between baseline and TOC. Wagenlehner et al. reported an MIC increase ( $\geq 4$-fold from baseline) in $8 / 393$ isolates (2\%) (86); Torres et al. reported a similar increase in $2 / 125$ isolates (1.6\%) (87). Several case reports have also described the emergence of CAZ-AVI resistance during therapy. Shields et al. reported resistance emergence (MIC $>8 \mathrm{mg} /$ liter) within a median of 15 days (range, 10 to 19) of treatment in $3 / 10(30 \%)$ patients among those treated with CAZ-AVI and experiencing microbiological failure (74). In another publication, Shields et al. (68) reported 3 cases of CAZ-AVI-resistant K. pneumoniae, cultured following CAZ-AVI treatment of 10 to 19 days. Performing whole-genome sequencing (WGS) on these isolates revealed new mutations in plasmid-borne $b \mathrm{la}_{\mathrm{KPC}-3}(68)$.

Another case report of a single patient showed mixed subpopulations of CAZ-AVI resistant KPC K. pneumoniae emerging following CAZ-AVI treatment (56). Though most cases of resistance to CAZ-AVI were described in K. pneumoniae sequence type 258 (ST258) $b l a_{\mathrm{KPC}-3}$-producing isolates, resistance has also been reported in $b / a_{\mathrm{KPC}-2}$ isolates, in other sequence types, with various mechanisms, and even without exposure to CAZ-AVI $(52,55)$. lannaccone et al. (109) reported 23 bacteremia cases of KPC K. pneumoniae treated with CAZ-AVI. Four cases had relapse of bacteremia: two of them with a CAZ-AVI-resistant isolate, one with an MIC increase from 2 to $8 \mu \mathrm{g} / \mathrm{ml}$, and one without relevant MIC variation. This corresponded to an incidence of $8.7 \%(2 / 23)$ for CAZ-AVI resistance (109).

Future clinical studies evaluating CAZ-AVI. No RCTs evaluated the use of CAZ-AVI versus BAT for CRE or carbapenem-resistant $P$. aeruginosa infections. Available data are limited by study design, selection bias, and common use of combination therapy. Currently, no RCTs are listed in ClinicalTrials.gov for CAZ-AVI. One listed retrospective study aims to compare CAZ-AVI versus BAT for CRE infections in adults. The study plans to include 344 patients and is currently recruiting patients (110). Since RCTs are unlikely to be performed, large observational studies are needed to fully describe efficacy of CAZ-AVI monotherapy, SAEs caused by the drug, and rates of resistance emergence during/following therapy.

\section{CEFTOLOZANE-TAZOBACTAM}

\section{Spectrum of Activity}

The combination of antipseudomonal cephalosporin- $\beta$-lactamase inhibitor ceftolozanetazobactam (TOL-TAZ) is active against common Gram-negative pathogens. It has doc- 
umented activity against ESBL-producing Enterobacterales, including E. coli carrying CTX-M-14 and CTX-M-15, MDR P. aeruginosa, as well as some anaerobes (Bacteroides fragilis and non-Bacteroides Gram negatives) and some Streptococcus spp. (111-121). However, TOL-TAZ has limited activity against ESBL-producing $K$. pneumoniae, carbapenemase-producing Enterobacterales, and anaerobic Gram-positive cocci (1, 5, 7, 11, 13-16) (Table 4).

Looking in more detail at specific bacterial targets, TOL-TAZ has shown particularly high activity against $P$. aeruginosa. Ceftolozane enhanced affinity for the PBPs of the $P$. aeruginosa; thus, it is significantly less affected by the changes in the porin permeability or efflux pumps (122). Data collected in the United States between 2011 and 2014 reported up to $97 \%$ susceptibility to TOL-TAZ in $P$. aeruginosa, including MDR and carbapenem nonsusceptible strains $(111,123)$. Equivalent data were reported from the United States between 2015 and 2017, showing 97.5\% susceptibility in P. aeruginosa

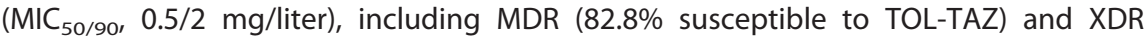
(82.9\% susceptible) isolates (124). Previous susceptibility rates are consistent with the results from the Asia-Pacific region and Canada, where the highest susceptibility to TOL-TAZ was in P. aeruginosa and Enterobacterales, including MDR and XDR isolates, and the combination had limited activity against bacteria with a CRE phenotype (125, 126). Sader et al. reported slightly diminished overall susceptibility rates in $P$. aeruginosa isolates from Europe, $86.3 \%$ (at $\leq 8 \mathrm{mg} /$ liter) and $84.5 \%$ ( $\leq 4 \mathrm{mg} /$ liter), respectively (114). However, in a recent Spanish nationwide study, 94.6\% from $P$. aeruginosa were susceptible to TOL-TAZ $\left(\mathrm{MIC}_{50 / 90}=1 / 2 \mathrm{mg} /\right.$ liter) (6). Also, a subsequent study from Shortridge et al. reported data about more than 6,000 Gram-negative isolates collected from pediatric patients in Europe and the United States, where susceptibility to TOL-TAZ was $94.6 \%$ and $97.4 \%$ of Enterobacterales and $P$. aeruginosa isolates, respectively (127).

A study by Tato et al. (115) showed that TOL-TAZ was highly active not only against MDR $P$. aeruginosa but also against $E$. coli, including wild-type, AmpC-like phenotype, and ESBL-producing isolates. The activity was diminished against ESBL-producing Klebsiella spp. $\left(\mathrm{MIC}_{50 / 90}, 4 / 16 \mathrm{mg} / \mathrm{liter}\right.$ ) and the combination was not active against carbapenemase-producing bacteria (MIC $\geq 64 \mathrm{mg} / \mathrm{liter}$ ) (115). Analyzing $E$. coli and Proteus mirabilis strains collected in China in 2017, the rates of susceptibility to TOL-TAZ reached $90.5 \%$ and $93.8 \%$, respectively. However, in the case of carbapenem-resistant $P$. aeruginosa, the sensitivity was $68 \%$, falling to $57.7 \%$ in $K$. pneumoniae and $1.9 \%$ in CRE isolates (128). Pazzini et al. (129) reported similar data: TOL-TAZ was active against $85 \%$ of ESBL-producing E.coli in contrast to $57.5 \%$ of ESBL-producing K. pneumoniae, but the majority of carbapenemase-producing Gram negatives (99.0\%) were not susceptible to TOL-TAZ (129). Recent susceptibility data reported from Portugal showed TOL-TAZ activity against $86.6 \%$ of Enterobacterales, including $99.4 \%$ of $E$. coli. The rates were falling to $71.5 \%$ in Klebsiella spp. and $70.4 \%$ in Enterobacter spp. The decreased susceptibility was mostly due to the ESBL- and carbapenemase-producing phenotypes (120). EUCAST, FDA, and CLSI breakpoints for the interpretation of the susceptibility of particular pathogens to TOL-TAZ are summarized in Table 2. For antimicrobial susceptibility data of various pathogens to TOL-TAZ, see Table 4.

\section{Resistance Rates and Mechanisms}

Various TOL-TAZ resistance mechanisms have been described, predominantly overexpression and mutations in the Ambler class $C \beta$-lactamase. $P$. aeruginosa produces a chromosomally encoded class $C$ cephalosporinase (Pseudomonas-derived cephalosporinase $[P D C]$ ) often responsible for the resistance to $\beta$-lactam antibiotics; but usually, PDCs are not efficient at hydrolyzing ceftolozane. Unlike tazobactam, ceftolozane inhibits PBPs, allowing tazobactam to target other serine $\beta$-lactamases (e.g., TEM-1) and ESBLs (e.g., CTX-M-15). Nevertheless, due to the emerging tendency in the acquisition of amino acid substitutions, PDCs can hydrolyze ceftolozane (130-134). In a study assessing the contribution of residues (V213, G216, E221, and Y223) in PDC-3 toward ceftolozane-tazobactam resistance, the E221K variant acquired an ability to efficiently 
hydrolyze ceftolozane causing alarming resistance (133). Already in 2014, a study by Cabot et al. reported the development of high-level resistance to the TOL-TAZ due to overexpression and structural modifications of AmpC; however, it occurred only in a $P$. aeruginosa with mutator (PAOMS, $\triangle$ mutS) background (135). Meanwhile, in a study by Sader et al. the majority of TOL-TAZ-resistant isolates carried a VIM-type MBL gene (114).

Assessing the emerging resistance following the exposure to TOL-TAZ, a study by Haidar et al. (130) reported that resistance developed in 14\% (3/21) of cases due to the de novo mutations, AmpC overexpression, and amino acid substitutions affecting the $\beta$-lactamase $\Omega$-loop. Resistance in the respective cases emerged on days 8 and 19 under the treatment and 2 weeks after completion of a 30-day treatment course, respectively (130). Skoglund et al. (131) reported a case where TOL-TAZ resistance in $P$. aeruginosa PA2428 developed without previous TOL-TAZ exposure. The patient had received antimicrobial therapy with ceftazidime, cefepime, meropenem, amikacin, and finally aztreonam but not particularly TOL-TAZ before the resistant isolate was cultured (131). Meanwhile, data from Spain showed that 10.6\% (5/47) of the patients developed resistance during the treatment of MDR $P$. aeruginosa infections with TOL-TAZ. The resistant strains were ST175 and ST179, and the underlying mechanisms for the resistance were a modification of $\mathrm{AmpC}$ and horizontally acquired $\beta$-lactamases in ST175 and the emergence of the extended-spectrum OXA $\beta$-lactamase OXA-14 in ST179 (132). A similar percentage was reported in a study by Jorgensen at al.: TOL-TAZ resistance was detected in 9.7\% (3/31) of MDR $P$. aeruginosa after 3, 7 and 8 days after the initiation of monotherapy with TOL-TAZ (136). However, in a recent case series report by Buonomo et al., including four adult patients with $P$. aeruginosa infection, there were no cases with the emergence of the resistance registered during the therapy with TOL-TAZ (137). Nevertheless, the number of cases was limited; therefore, additional studies and surveillance of the emerging resistance following the exposure to TOL-TAZ are needed.

\section{PK-PD Characteristics}

The pairing of ceftolozane with tazobactam illustrates the feasibility of combining a $\beta$-lactam and $\beta$-lactamase inhibitor that are not perfectly matched pharmacokinetically. Ceftolozane and tazobactam share similar protein binding values (as summarized in Table 5) but differ in half-life and metabolic disposition. Ceftolozane is excreted unchanged, and less than $20 \%$ of tazobactam is metabolized to an inactive M1 metabolite. Unmetabolized tazobactam, the M1 metabolite, and ceftolozane are all excreted renally $(4,138)$. Similar to that for avibactam, preclinical studies identified the duration of time above a threshold value ( $\left.\% T_{>\text {threshold }}\right)$ as the PK-PD index that best described the activity of tazobactam when it was administered in combination with ceftolozane.

In vitro hollow-fiber studies illustrated the dependence of $\% T_{>\text {threshold }}$ on the degree of $\beta$-lactamase expression; high expression of CTX-M-15 was associated with a higher threshold value for suppression of bacterial growth (4). PK-PD data from a neutropenic murine thigh infection model was used to identify a threshold value of $1 \mu \mathrm{g} / \mathrm{ml}$ in a collection of $\beta$-lactamase-producing $K$. pneumoniae and E. coli. A target of $65.6 \% T$ of $>1 \mu \mathrm{g} / \mathrm{ml}$ was used for PTA analysis. Consistent with other $\beta$-lactams, $\% T_{>\text {MIC }}$ was identified as the index that best correlated with antimicrobial activity of ceftolozane against non- $\beta$-lactamase-producing Enterobacterales and $P$. aeruginosa. A plasma target of $32.2 \%$ associated with a $1-\log _{10}$ CFU decrease was initially proposed for PTA analysis; however, final analysis relied on a more conservative target of $40 \%$ $T_{>\text {MIC }}$ associated with $2-\log _{10}$ CFU reduction $(4,138)$.

For CUTI and CIAl indications, the approved dosing regimen is $1 \mathrm{~g}$ ceftolozane and $0.5 \mathrm{~g}$ tazobactam administered every $8 \mathrm{~h}$ as a 1-h i.v. infusion. (138) A higher dose of $2 \mathrm{~g}$ ceftolozane and $1 \mathrm{~g}$ tazobactam is indicated for the treatment of hospital-acquired bacterial pneumonia/ventilator-associated pneumonia (HABP/VABP) to account for the lower exposures of ceftolozane and tazobactam observed in the epithelial lining fluid 
(ELF) than in plasma. As with ceftazidime-avibactam, the dosage adjustments required in patients with renal impairment $(\mathrm{CrCL} \leq 50 \mathrm{ml} / \mathrm{min})$ are consistent with the predominant renal elimination of the combination. While there are no formalized dosing recommendations in children, dose selection based on matching pediatric exposures to adult exposures in approved doses suggest that for clAI and CUTI, children $\geq 12$ years old may receive the approved adult dose (139). For pediatric patients aged $<12$ years, $20 \mathrm{mg} / \mathrm{kg}$ body weight ceftolozane and $10 \mathrm{mg} / \mathrm{kg}$ tazobactam administered as a $1-\mathrm{h}$ infusion every $8 \mathrm{~h}$ is proposed for the phase 2 trials.

\section{Clinical Data}

Approval date and indications. TOL-TAZ is EMA and FDA approved. It was first approved by FDA in 2014 for use in adults with CIAI (in combination with metronidazole) and CUTI, including pyelonephritis. The dosage approved for these indications was $1.5 \mathrm{~g}$ every $8 \mathrm{~h}$. It was later approved in 2019 for the treatment of adults with HABP/VABP at a dosage of $3 \mathrm{~g}$ every $8 \mathrm{~h}$. It has not yet been approved for use in pediatric patients (138).

Randomized controlled trials evaluating TOL-TAZ for any infection. See Table 8 for a data summary from RCTs. To date, four RCTs have been published comparing TOL-TAZ versus other regimens, including versus meropenem for nosocomial pneumonia (140) and CIAI (141, 142) and versus levofloxacin for CUTI (143).

A meta-analysis by Cheng et al. (144) compiled three RCTs evaluating TOL-TAZ for CUTI or ClAI, including, overall, 2,198 patients. Clinical cures at TOC and microbiological responses were similar between study arms. Specifically, in the cUTI trial, higher microbiological response rates with TOL-TAZ were demonstrated. Nevertheless, addressing separately the Gram-positive pathogens, TOL-TAZ had a significantly lower eradication rate. No significant difference between groups was demonstrated in AEs, including SAEs and those resulting in discontinuation of the study drug (144).

An additional RCT evaluated TOL-TAZ versus meropenem for the treatment of nosocomial pneumonia (726 patients). No significant difference between study arms was demonstrated for any of the efficacy and safety outcomes, though SAEs were nonsignificantly more common with TOL-TAZ (42.1\% versus $35.9 \%)$. This trial used a high dose of TOL-TAZ ( $3 \mathrm{~g}$ every $8 \mathrm{~h}$ compared to $1.5 \mathrm{~g}$ every $8 \mathrm{~h}$ administered in other trials). Most common AEs in this study included gastrointestinal adverse events (2\%), abnormal liver function tests (5\%), and Clostridioides difficile infection (2\%) (140). No significant difference in SAEs was demonstrated in the other three RCTs described above, using the dose of $1.5 \mathrm{~g}$ every $8 \mathrm{~h}$ (141-143) (see Table 9 for dosages of new BLBLIs).

Clinical data on the efficacy of TOL-TAZ for infections caused by specific pathogens. (i) Pseudomonas aeruginosa. Twelve studies reported data on the efficacy of TOL-TAZ in $P$. aeruginosa infections (see Table 10), two were comparative versus polymyxin or aminoglycoside for XDR $P$. aeruginosa infections $(145,146)$. Overall, in these studies, mortality was $17.6 \%$ (110/624): $20.1 \%$ among MDR/XDR infected patients (105/523). Clinical and microbiological success rates were $76.6 \%(477 / 623)$ and $75.6 \%$ (480/635), respectively, for any P. aeruginosa, and 73.4\% (380/512) and 74.2\% (370/508), respectively, among MDR/XDR infected patients.

All these studies included patients treated with a dose of either $1.5 \mathrm{~g}$ every $8 \mathrm{~h}$ or $3 \mathrm{~g}$ every $8 \mathrm{~h}$, with the high dose usually administered for cases of pneumonia, osteomyelitis, and abscess. Gallagher et al. reported the use of high-dose $(3 \mathrm{~g}$ every 8 h) TOL-TAZ in 97/205 patients, without improved outcomes among these patients (147).

(ii) Extended spectrum $\boldsymbol{\beta}$-lactamase Enterobacterales. Three RCTs comparing TOLTAZ versus meropenem did not report a difference in clinical cure between trial arms for patients with ESBL infections. In one trial evaluating TOL-TAZ versus levofloxacin for cUTI, clinical cure rates were higher with TOL-TAZ (143). Huntington et al. (148) performed a post hoc analysis of data from this RCT. This analysis included 212 patients with levofloxacin-resistant pathogens at baseline; 186 had Enterobacterales infection, 


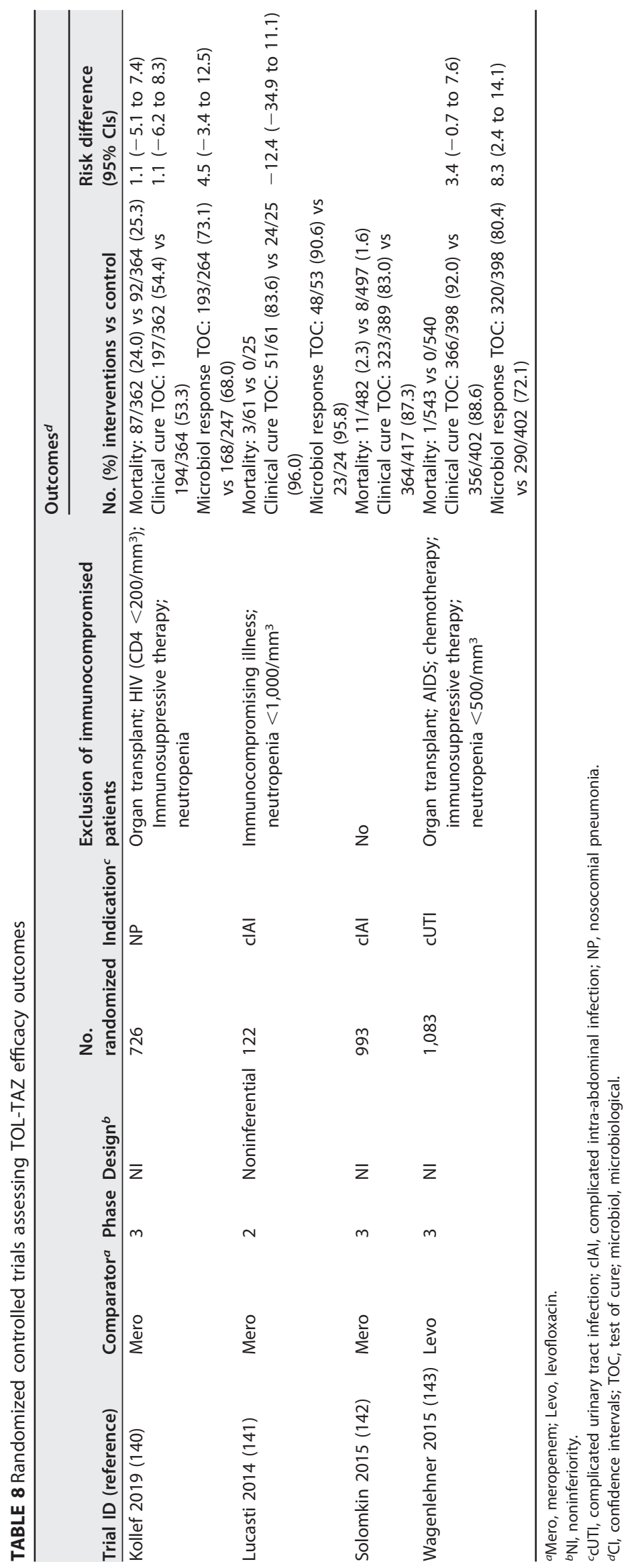




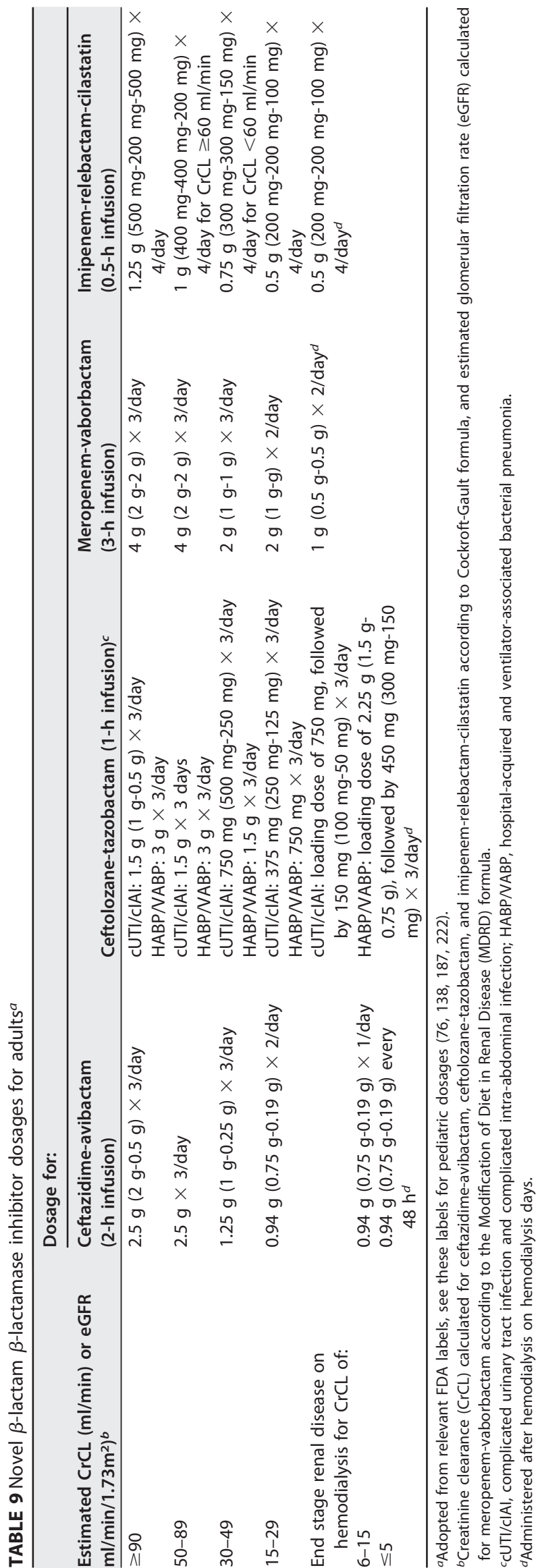




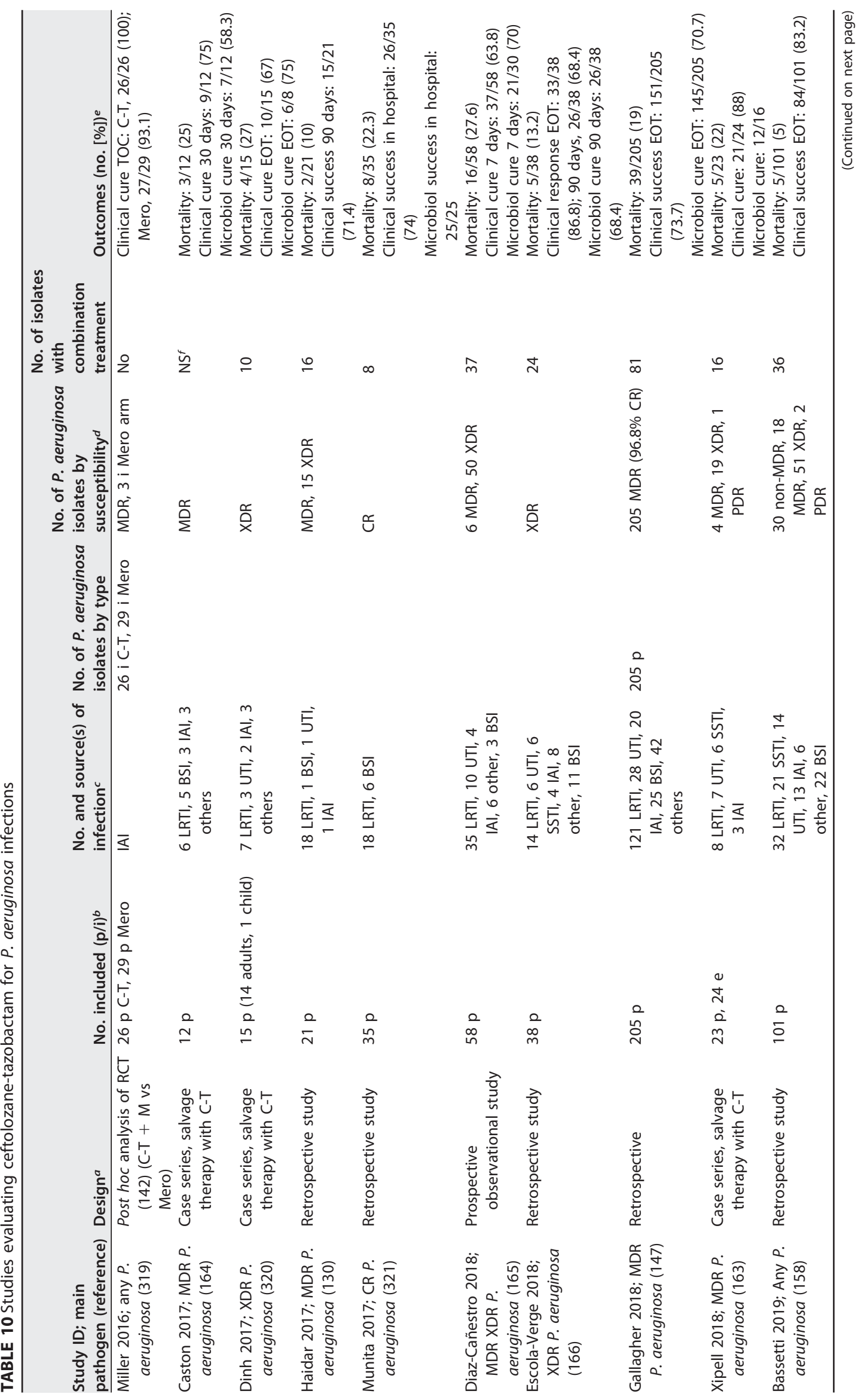




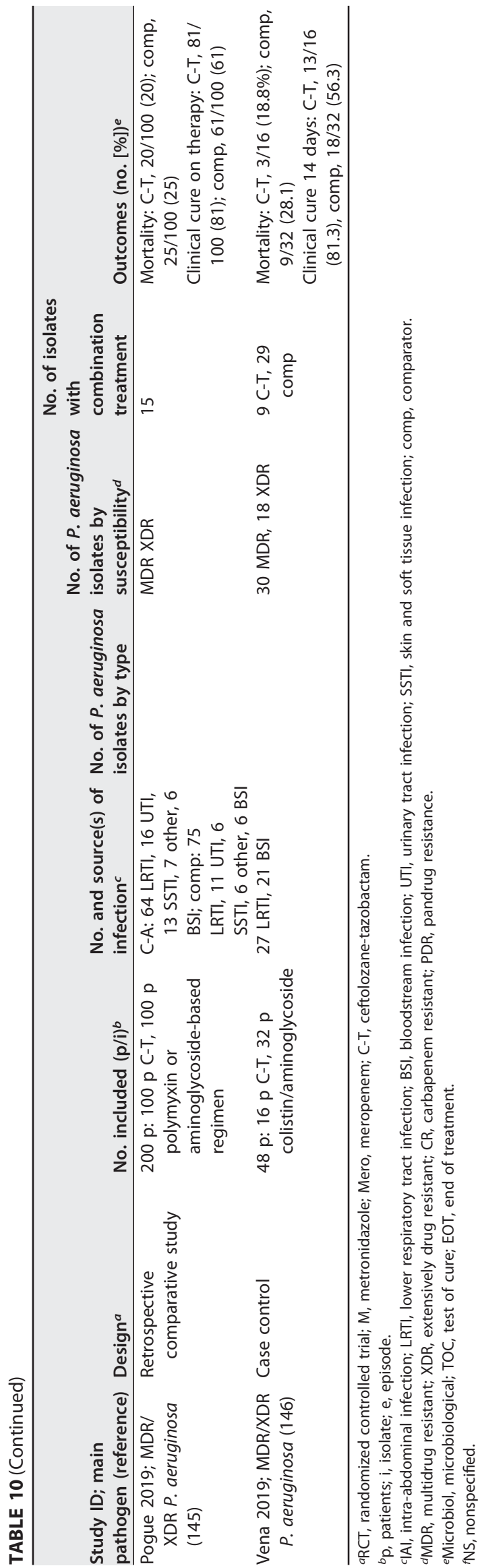


and 85 were ESBL positive. Among patients with ESBL infection, both clinical and microbiological cure rates were significantly higher in the TOL-TAZ arm (148) (for details see Table 11).

Two additional studies were noncomparative prospective interventional studies, aiming to evaluate the efficacy and safety of TOL-TAZ for the treatment of CUTI (149) and CIAI (150). Overall, 18 patients with ESBL infection were included, with no mortality cases. All 5 patients with clAl had clinical and microbiological response. Five of 13 (38.5\%) patients with CUTI had microbiological response; this rate was low, at approximately one-half of that of non-ESBL infections (Table 11).

Clinical use of TOL-TAZ in special patient populations. (i) Pediatric population. To date, only one phase 1 study was published evaluating the safety of TOL-TAZ in pediatric patients. This study included 37 children in various age groups and showed no mortality cases, no serious clinical adverse events, and no clinically significant laboratory abnormalities (151). Two phase 2 trials in children are ongoing and currently recruiting patients. One trial aims to compare TOL-TAZ with meropenem for treatment of CUTI in 120 children (152), the other will compare TOL-TAZ plus metronidazole versus meropenem for an additional 120 children with clAI (153). In addition, few case reports have described successful clinical use of TOL-TAZ in children with $P$. aeruginosa infections (154-156).

(ii) Chronic renal failure. Patients with severe impairment of renal function were excluded from RCTs evaluating TOL-TAZ. Kullar et al. (157) summarized data on patients with moderate renal impairment ( $\mathrm{CrCL} 30$ to $50 \mathrm{ml} / \mathrm{min}$ ) from RCTs by Solomkin et al. (142) (36 patients) and Wagenlehner et al. (59 patients) (143). In both trials, moderate renal impairment, age $\geq 65$ years, and diabetes mellitus were risk factors for clinical failure. Patients with moderate renal impairment in the CIAI trial (142) but not the CUTI trial (143) had lower clinical response rates with TOL-TAZ (48\% [11/23]) than with meropenem (69\% [9/13]). Dosage of TOL-TAZ for moderate renal impairment in this trial was $750 \mathrm{mg}$ every $8 \mathrm{~h}$.

Kollef et al. (140) reported similar clinical cure rates between TOL-TAZ and comparator in the subgroup of patients with reduced renal clearance, though a trend for lower clinical response is reported for moderate-severe renal impairment (38.4\% TOL-TAZ versus $44.6 \%$ meropenem) (140).

Bassetti et al. (158) reported retrospectively the results of 101 patients treated with TOL-TAZ for different $P$. aeruginosa infections, mainly, nosocomial pneumonia and skin and soft tissue infections. Multivariate analysis of risk factors for clinical failure demonstrated chronic renal replacement therapy (CRRT), in addition to sepsis, as a risk factor for failure. The TOL-TAZ dosage used for CRRT patients was $1.5 \mathrm{~g}$ every $8 \mathrm{~h}$. The authors suggested considering therapeutic drug monitoring in this setting (158). Other suggested solutions for CRRT patients are raising the dosage to $3 \mathrm{~g}$ every $8 \mathrm{~h}$ (159) or using continuous infusion of $6 \mathrm{~g}$ in $24 \mathrm{~h}$ (160).

(iii) Diabetes mellitus. Popejoy et al. (161) reported outcomes of 245 diabetic patients compared to 1,802 nondiabetic patients from two RCTs $(142,143)$. Patients with diabetes had lower clinical response overall; however, no difference between TOL-TAZ and comparator was demonstrated (161).

(iv) Immunocompromised. RCTs and prospective studies evaluating TOL-TAZ did not include immunocompromised patients. All the retrospective studies (Tables 10 and 11 ) reported on including immunocompromised patients (transplant recipients, $8 \%$ to $43 \%$ of included patients; patients with malignancy or other immunosuppression, $10 \%$ to $34 \%$ of included patients); however, few reported outcomes for these patients specifically.

(v) Elderly patients. Three RCTs reported outcomes for 639 elderly patients, none of them demonstrating a significant difference in clinical cure rates between TOL-TAZ and comparator (140, 142, 143). As described above, Kullar et al. reported higher risk for clinical failure in patients aged 65 years and older treated with TOL-TAZ for CUTI or CIAI (157). 


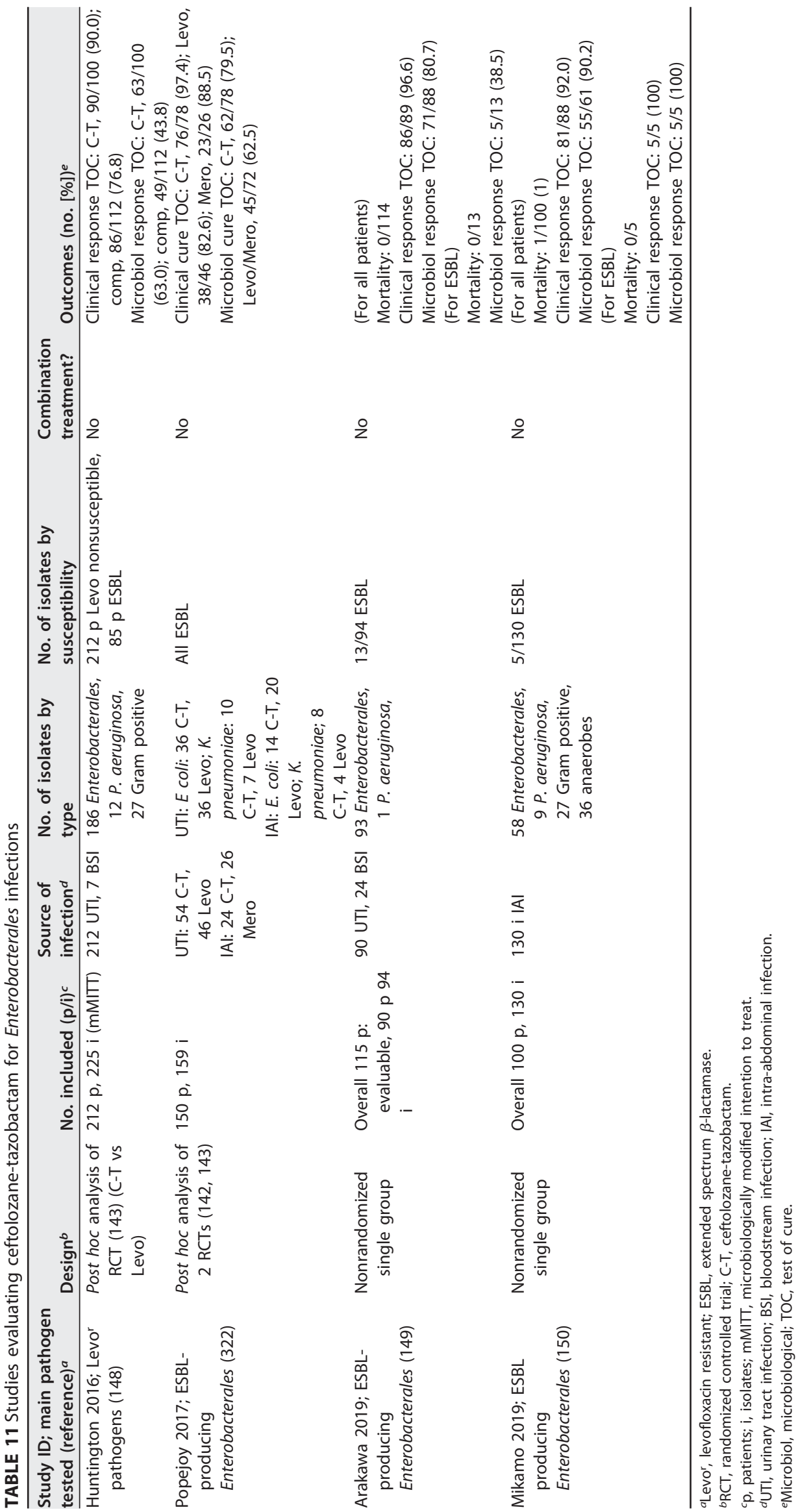


Clinical use of TOL-TAZ for specific bacteria. No clinical studies tested TOL-TAZ against Burkholderia spp.; however, some in vitro data are available.

(i) In vitro activity against specific bacteria. TOL-TAZ has good in vitro activity against Burkholderia cepacia spp., with $89 \%$ of the strains susceptible based on CLSI breakpoints for $P$. aeruginosa in one study (105). In the same study, TOL-TAZ was shown to be less active against $B$. gladioli spp., and have some activity against $S$. maltophilia, with an advantage over ceftazidime for this pathogen (105). No advantage over ceftazidime was demonstrated against Acinetobacter spp. or Elizabethkingia spp. (162).

Safety data. In RCTs assessing TOL-TAZ, rates of AEs in general were similar between TOL-TAZ and the comparator. Most common AEs included gastrointestinal AEs, $C$. difficile infection, headaches, pyrexia, and abnormal liver function tests. In the only trial using high-dose TOL-TAZ for pneumonia ( $3 \mathrm{~g}$ every $8 \mathrm{~h}$ ), there was a trend for higher rate of SAEs (140).

In two prospective studies using usual dose of TOL-TAZ (1.5 g every $8 \mathrm{~h}$ ), any AEs were reported in $58 \%$ to $62 \%$, and drug-related AEs were reported in $17.5 \%$ to $19 \%$. Most common AEs were gastrointestinal events, insomnia, and abnormal liver function tests $(149,150)$.

Pogue et al. (145) reported use of high-dose TOL-TAZ in $63 \%$ of 100 patients with MDR/XDR $P$. aeruginosa infections. Safety data were not separately addressed; however, among clinical outcomes, six cases of acute kidney injury and four cases of $C$. difficile infection during therapy were reported (145). Bassetti et al. (158) reported, overall, three drug-related AEs ( ash, gastrointestinal symptoms, and liver function test abnormalities) among 101 patients; $30 \%$ of them received high-dose TOL-TAZ.

Emergence of resistance to TOL-TAZ during treatment. RCTs evaluating TOL-TAZ reported baseline resistance to TOL-TAZ, but none tested for emergence of resistance. Several nonrandomized studies reported resistance development during or after TOLTAZ therapy, with various rates. Among 101 patients treated with TOL-TAZ for various $P$. aeruginosa infections, TOL-TAZ resistance was detected in 3 patients (3.0\%) during or following treatment (158). Xipell et al. demonstrated similar rates with 1 of 23 patients developing a resistant strain (163). In contrast, other studies reported higher rates of resistance emergence during or in subsequent cultures, up to $11 \%$ to $17 \%(130$, 164-166). Mutations leading to such resistance involved overexpression or structural modification of AmpC (including T96I, E247K, and $\Omega$-loop deletions, amino acid replacement in residue E247 [E247G], and F147L mutation) or involved the OXA-10 enzyme $(132,165)$. Factors suggested to be associated with selection of resistant strains include time above MIC of $10 \%$ to $30 \%$, clinical failure, and microbiological failure (163, 165).

Future clinical studies evaluating TOL-TAZ. In addition to two ongoing trials evaluating TOL-TAZ in pediatric populations $(152,153)$, two other trials are currently recruiting participants. A phase II RCT evaluates TOL-TAZ versus standard therapy as empirical therapy for 100 neutropenic febrile patients (167). Another RCT is planned to recruit 268 patients with clAI for the comparison of TOL-TAZ plus metronidazole versus meropenem (168). An additional trial was posted on ClinicalTrials.gov in January 2020. This is an open label randomized controlled trial comparing ceftolozane-tazobactam versus meropenem for the treatment of bloodstream infections caused by ESBL- and AmpC-producing Enterobacterales. The sample size is calculated at 630 patients and the primary outcome is 30-day mortality (169).

As in the case of CAZ-AVI, data regarding the use of TOL-TAZ for MDR infections are limited by design, selection of patients for treatment with the new drug, and number of patients included. Further observational studies are needed.

\section{MEROPENEM-VABORBACTAM \\ Spectrum of Activity}

Vaborbactam was developed to restore the activity of $\beta$-lactams against $\beta$-lactamase-producing Gram-negative bacteria, particularly to inactivate $K$. pneumoniae carbapenemases (KPCs). (170) A combination of meropenem-vaborbactam 
(MER-VAB) has documented activity against class $C \beta$-lactamases, a variety of class $A$ $\beta$-lactamases, including CTX-M, SHV, TEM, SME, and NMC-A, KPC-producing isolates, and $\mathrm{FRI}-1$ as well as BKC-1 carbapenemases found in K. pneumoniae and E. cloacae (170-178). Vaborbactam also restored the activity of meropenem against class $A$ and class $C \beta$-lactamase-producing $K$. pneumoniae strains with reduced permeability due to porin mutations. MER-VAB does not inhibit class D (OXA-48) or class B carbapenemases $(174-176,179)$. The activity of vaborbactam against $\beta$-lactamases is summarized in Table 1.

Addressing particular data, in a study by Lapuebla et al. evaluating MER-VAB activity against KPC-producing Enterobacterales, the combination inhibited 98.5\% (131/133) of isolates at the meropenem concentration of $1 \mathrm{mg} /$ liter (174). It should be noted that in all studies, a fixed vaborbactam concentration of $8 \mathrm{mg} / \mathrm{liter}$ was used if not stated differently. A study by Castanheira et al. (171) including 315 serine carbapenemaseproducing Enterobacterales showed that meropenem alone at $\leq 1 \mathrm{mg} / \mathrm{liter}$ and $\leq 2 \mathrm{mg}$ / liter inhibited only $2.2 \%$ and $7.3 \%$ of the bacteria, respectively. The combination ( $\leq 2 \mathrm{mg} /$ liter of MER-VAB) inhibited $\geq 96.5 \%$ of the isolates (171).

Data from a study analyzing 10,426 Enterobacterales collected worldwide during 2014 showed that MER-VAB inhibited $99.3 \%$ of isolates at a meropenem MIC of $\leq 2$ mg/liter (172). In a subsequent study on 11,559 Enterobacterales isolates collected during 2015, MER-VAB inhibited $99.3 \%$ of all strains and $99.5 \%$ of KPC producers at the FDA susceptibility breakpoint of $\leq 4 / 8 \mathrm{mg} /$ liter. The combination had limited activity against MBL-producing bacteria (including 49 NDM, 1 IMP-64, and 2 VIM) and/or carbapenem-hydrolyzing class D enzymes (47 OXA-48/-232), which is consistent with the previously reported data (178). In an in vitro study by Hackel et al. (180), 99.0\% (981/991) of KPC-positive (OXA-48- and MBL-negative) Enterobacterales had MER-VAB MICs of $\leq 4 \mu \mathrm{g} / \mathrm{ml}$. Vaborbactam lowered the $\mathrm{MIC}_{90}$ of meropenem from $>32$ to $1 \mathrm{mg} /$ liter (180). Kinn et al. (181), assessing in vitro activity of MER-VAB against carbapenem-resistant Enterobacterales (CRE) isolates, reported that MIC decreased by 128-fold on average compared with that for exposure to meropenem alone. The authors also highlighted that vaborbactam significantly decreased meropenem MIC in both non-KPC-producing and KPC-producing CRE despite that previous studies have demonstrated decreased vaborbactam activity in non-KPC-producing CRE. The authors assume that this finding is due to the absence of NDM and OXA-48 isolates in the study sample population (181).

In a recently published study assessing the activity of MER-VAB against pneumoniacausing bacteria (3,193 P. aeruginosa and 4,790 Enterobacterales) isolated between 2014 and 2018 from patients in U.S. hospitals, 99.9\% of Enterobacterales (including CRE) and $89.5 \%$ of $P$. aeruginosa were susceptible to MER-VAB (182). Evaluating activity against carbapenem-resistant Enterobacterales in an in vitro hollow-fiber model during $>32-\mathrm{h}$ exposure, MER-VAB ( $2 \mathrm{~g} / 2 \mathrm{~g})$ showed bactericidal activity against $K$. pneumoniae, $E$. cloacae, and E. coli strains, MICs were up to $8 \mathrm{mg} / \mathrm{liter}$ (183).

Data from the clinical practice are limited, but in 2019, Athans et al. (184) reported a clinical case of a liver transplant recipient developing bacteremia with a KPCproducing $K$. pneumoniae isolate becoming resistant to CAZ-AVI after 33 days of CAZ-AVI monotherapy; the isolate harbored a D179Y mutant $b / a_{\mathrm{KPC}-2}$ gene. The infection resolved after treatment with MER-VAB, indicating that this combination could be important under conditions of emerging CAZ-AVI resistance (184). The respective EUCAST-, FDA-, and CLSI-approved breakpoints for the interpretation of the susceptibility of particular pathogens to MER-VAB are summarized in Table 2. For antimicrobial susceptibility data of various pathogens to MER-VAB, see Table 4.

\section{Resistance Rates and Mechanisms}

Several studies have reported that the MER-VAB combination is not active against bacteria producing MBL or OXA carbapenemases (174-176). Vaborbactam also lacked the activity against Gram-negative bacteria with porin mutations combined with overexpression of efflux pumps. A decreased potency of vaborbactam against strains 
affected by the inactivation of OmpK35 and OmpK36 and strains overexpressing AcrA have been documented $(170,176,178,185)$. In a previously mentioned study by Castanheira et al., MER-VAB-resistant isolates produced MBL (MIC, 16 to $\geq 64 \mathrm{mg} /$ liter) or had decreased expression of ompK37 porin and/or hyperexpression of the AcrABTolC efflux system (MIC, $16 \mathrm{mg} / \mathrm{liter}$ ) (171). A study by Lapuebla et al. also identified bacteria resistant to MER-VAB due to diminished OmpK35 and OmpK36 expression, where MICs for MER-VAB were 8- to 16-fold higher than for isolates with the same $\beta$-lactamases but without porin changes (174). A surveillance study by Castanheira et al. described increased MER-VAB MIC (>8 mg/liter) in bacteria with a mutation in ompK35 or harboring $b l a_{\mathrm{KPC}}$ with bla $a_{\mathrm{OXA}-48 \text {-like }}$ or bla $a_{\mathrm{NDM}-1}(186)$.

\section{PK-PD Characteristics}

Both vaborbactam and meropenem display low plasma protein binding ( $\sim 33 \%$ and $\sim 2 \%$, respectively) and comparable volumes of distribution at steady state $\left(V_{\mathrm{ss}}\right)$ and half-lives (Table 5). Approximately $28 \%$ of meropenem is hepatically hydrolyzed to an inactive open lactam metabolite; both the parent and metabolite are excreted renally. Vaborbactam is also excreted unchanged in urine $(187,188)$.

The PK-PD targets for both meropenem and vaborbactam were defined based on meropenem MICs with a fixed concentration of $8 \mathrm{mg} /$ liter vaborbactam. The magnitude of $\% f T_{>M I C}$ required for maximal meropenem efficacy was determined for KPCproducing Enterobacterales as $30 \%$ to $45 \% \mathrm{fT}_{>\mathrm{MIC}}$. For vaborbactam, $\mathrm{fAUC}_{\mathrm{O}-24} / \mathrm{MIC}$ was the index identified to describe the inhibitory activity of vaborbactam in preclinical studies against a fixed exposure of meropenem (i.e., the human-equivalent dose of $2 \mathrm{~g}$ infused over $3 \mathrm{~h}$ every $8 \mathrm{~h}$ ). A target $\mathrm{AAUC}_{0-24} / \mathrm{MIC}$ of 38 derived in murine thigh infection models using KPC-producing Enterobacterales was subsequently used in PTA analyses to support the proposed dosing and susceptibility breakpoints (14).

While the highest approved dose for meropenem is $1 \mathrm{~g}$ every $8 \mathrm{~h}$ administered as a 15- to 30- min infusion, a 2-g meropenem dose and a longer duration of infusion (3 h) were evaluated in a PTA analysis and a preclinical hollow-fiber infection model (183). The use of high-dose meropenem delivered over an extended infusion is supported by clinical experience in the treatment of infections due to carbapenem-resistant Enterobacterales and other meropenem-resistant strains (14). Consequently, the approved dose for this combination is $2 \mathrm{~g}$ each of meropenem and vaborbactam infused over 3 $\mathrm{h}$ every $8 \mathrm{~h}$. Renal impairment status was the only intrinsic factor warranting dosage adjustment based on estimated glomerular filtration rate (eGFR; ml/min/1.73 $\mathrm{m}^{2}$ ). Currently, there are no recommendations for the use of this combination in pediatrics.

\section{Clinical Data}

Approval date and indications. MER-VAB is EMA and FDA approved. It was first approved by FDA in 2017 for use in adults with CUTI, including pyelonephritis. The dosage approved for these indications was $4 \mathrm{~g}$ every $8 \mathrm{~h}$. It has not yet been approved for use in pediatric patients (187).

Randomized controlled trials evaluating MER-VAB for any infection. The TANGO I phase 3 RCT randomized 550 adult patients to either MER-VAB or piperacillintazobactam (PIP-TAZ) for the treatment of CUTI, including pyelonephritis (189). Patients with severe renal or hepatic impairment, septic shock, or immunosuppression were excluded. Three hundred seventy-four patients were included in the microbiological intention-to-treat population (MITT); 85\% of them had Enterobacterales as the causative pathogen. For the MITT population, overall success at end of intravenous treatment, defined as clinical cure or improvement and microbial eradication, was 189/192 (98.4\%) in the MER-VAB arm versus $171 / 182(94.0 \%)$ in the PIP-TAZ arm, with a significant advantage to MER-VAB (risk difference [RD], 4.5; 95\% confidence interval [CI], 0.7 to 9.1). No significant difference was demonstrated for the outcome of clinical cure alone (RD, 2.8; $95 \% \mathrm{Cl},-0.7$ to 7.1 ). At test of cure, no difference between MER-VAB and PIP-TAZ was demonstrated in either overall success or clinical cure. Microbiological eradication was significantly better with MER-VAB at end of intravenous treatment but not at test 
of cure. Subgroup analysis for the outcome of overall success at the end of intravenous treatment showed significantly higher success rates with MER-VAB in patients aged 65 years and older, women, patients without sepsis or bacteremia, and patients with higher Charlson comorbidity scores ( $\geq 3$ ).

No difference between MER-VAB and PIP-TAZ was demonstrated for any AEs (106/ 272 [39.0\%] MER-VAB versus 97/273 [35.5\%] PIP-TAZ group) or SAEs (11/272 [4.0\%] MER-VAB versus 12/273 [4.4\%] PIP-TAZ); however, AEs resulting in drug discontinuation were more common with PIP-TAZ (7/272 [2.6\%] MER-VAB versus 14/273 [5.1\%] PIPTAZ). Two patients in each group died. Most common AEs with MER-VAB were headache, diarrhea, phlebitis, and abnormal liver function tests (189).

MER-VAB in specific populations. (i) Pediatric population. A phase 1 study for dose finding, pharmacokinetics, and safety of MER-VAB in children with serious bacterial infections is currently ongoing (190). It is planned to enroll 60 patients, who will be receiving a single dose MER-VAB infused over $3 \mathrm{~h}$, at the following dosage regimens: age 12 to $<18$ years, $40 \mathrm{mg} / \mathrm{kg}$ meropenem and $40 \mathrm{mg} / \mathrm{kg}$ vaborbactam, or $2 \mathrm{~g}$ meropenem $2 \mathrm{~g}$ vaborbactam for subjects $\geq 50 \mathrm{~kg}$ in weight; for age 2 to $<6$ years, $60 \mathrm{mg} / \mathrm{kg}$, or $2 \mathrm{~g}$ meropenem $2 \mathrm{~g}$ vaborbactam for subjects $>33 \mathrm{~kg}$ in weight (190).

A single case report described a 4-year-old child with KPC-producing $K$. pneumoniae bacteremia treated with MER-VAB at a dose of $40 \mathrm{mg} / \mathrm{kg}$ every $6 \mathrm{~h}$ infused over $3 \mathrm{~h}$ (191). The patient received this therapy as monotherapy for 14 days with no evidence of treatment emergent adverse events and with good clinical outcome and clearance of bacteremia. The pharmacodynamic target of time above MIC of $\geq 40 \%$ was achieved for $100 \%$ of the dosing interval (191).

(ii) Cancer patients. Viale et al. (192) performed a subgroup analysis of 15 cancer patients with CRE infection who were included in the TANGO II trial (193) (MER-VAB versus BAT for suspected CRE infections). Among these patients, 8 patients that were treated with MER-VAB (versus 7 with BAT) had significantly lower mortality, fewer AEs, and higher clinical and microbiological cure rates (192).

Clinical use of MER-VAB for specific bacteria. (i) CRE. The TANGO II phase 3 RCT randomized 77 adult patients with CRE infections to receive either MER-VAB (52 patients [p]) versus BAT (25 p) (193). Patients with confirmed infection due to Ambler class B or D Enterobacterales were excluded. The population with a confirmed CRE isolate included 32 MER-VAB patients and 15 BAT patients (most of them treated with various combinations, including 2 to 3 of carbapenem, polymyxin, aminoglycoside, and/or tigecycline). More than one-half of the patients in each group had either bacteremia or CUTI; other included sources were CIAI and HABP/VABP. Thirty four percent of patients in the MER-VAB arm and $53 \%$ in the BAT arm were immunocompromised; $22 \%$ and $27 \%$, respectively, had baseline $\mathrm{CrCLs}$ of $<50 \mathrm{ml} / \mathrm{min}$. K. pneumoniae was the most common pathogen (87\%), mostly KPC-producing species (73\%). Clinical cure at EOT and TOC was significantly higher with MER-VAB than with BAT. The microbiological cure was nonsignificantly higher in the MER-VAB group and mortality was nonsignificantly lower (193) (see Table 12).

Subgroup analysis showed improved clinical cure with MER-VAB in patients aged 65 years and older, patients with a Charlson score of $\geq 4$, and immunocompromised patients. Any AEs were less frequent with MER-VAB (42/50 [84.0\%] MER-VAB versus 23/25 [92.0\%] BAT), and a similar trend was observed for SAEs (17/50 [34.0\%] versus $11 / 25$ [44.0\%]). Decreased nephrotoxicity was shown with MER-VAB. The most common AEs with MER-VAB were diarrhea, hypotension, anemia, and hypokalemia (193).

Bassetti et al. (194) performed a post hoc analysis of this RCT (193), analyzing 22 MER-VAB patients and 15 BAT patients without prior antimicrobial failure. Significantly lower mortality and higher rates of clinical and microbiological cure were demonstrated with MER-VAB (Table 13).

Lai et al. (195) compiled the two TANGO trials $(189,193)$ in a meta-analysis and found no difference between MER-VAB and comparator for any of the efficacy or safety outcomes. Shields et al. (196) conducted a prospective noncomparative study, using MER-VAB as treatment of choice for 20 consecutive patients with confirmed or sus- 


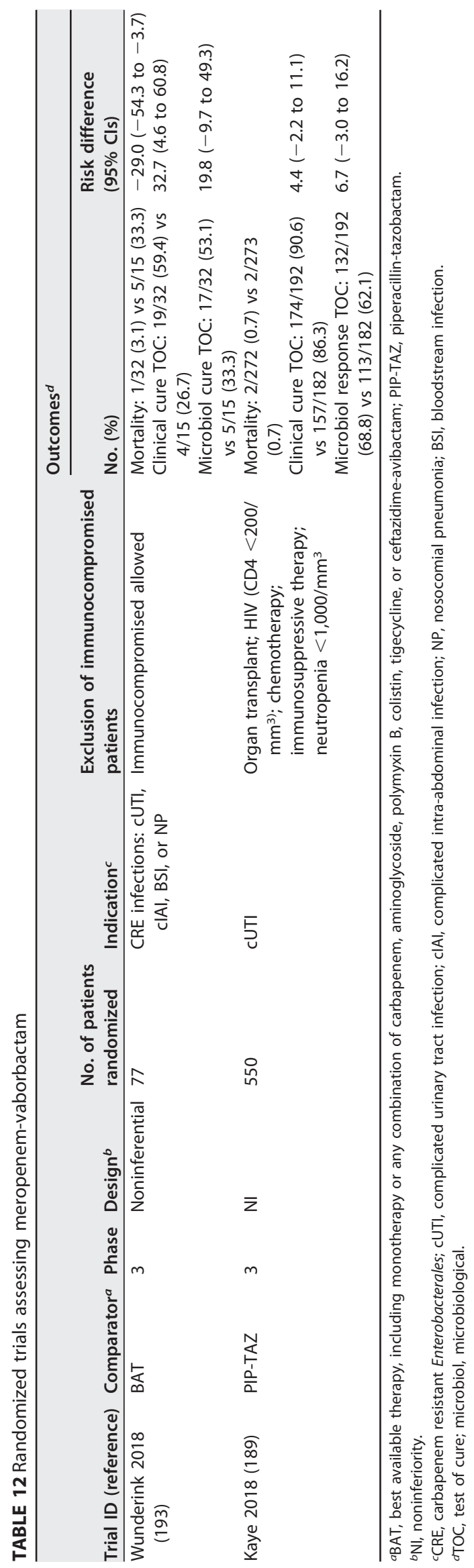




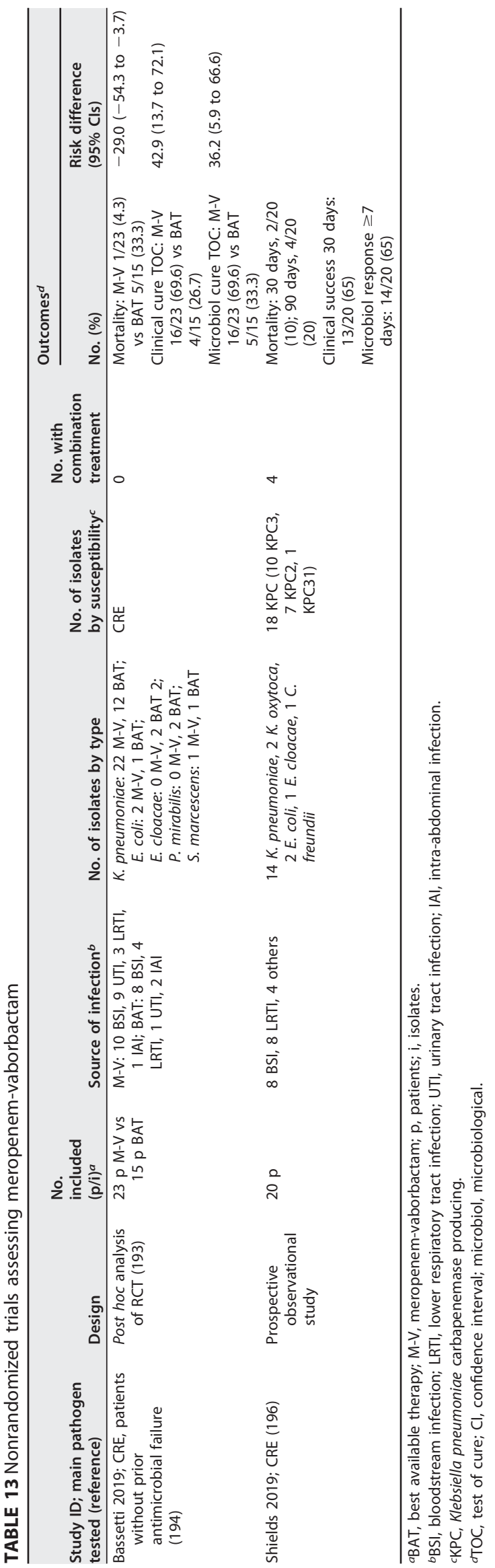


pected CRE infections. These patents were mostly intensive care unit (ICU) patients (70\%), with $35 \%$ of them requiring renal replacement therapy at infection onset (6 intermittent hemodialysis, 1 continuous renal replacement therapy [CRRT]). Dosages of the drug used were according to the manufacturer, with $2 \mathrm{~g}$ every $8 \mathrm{~h}$ administered for CRRT patients. Mortality at 30 and 90 days was $2 / 20$ and $4 / 20$, respectively, clinical cure was $65 \%$ at 30 days, and after $\geq 7$ days of treatment, microbiological failure was reported in 6 patients (35\%). Higher APACHE II scores were associated with clinical failure. Need for RRT and type of infection did not influence cure rates (196).

A recent case report described an HIV patient with KPC-producing Serratia marcescens and Enterobacter aerogenes bacteremia. The patient failed treatment with CAZ$\mathrm{AVI}$ and was switched to MER-VAB and also underwent a source control procedure. He was successfully treated with MER-VAB for 14 days and discharged from hospital (197). Kufel et al. (198) reported on a patient with a carbapenem-resistant K. pneumoniae prosthetic joint infection who was treated as first line with MER-VAB ( $2 \mathrm{~g}$ over $3 \mathrm{~h}$ every $8 \mathrm{~h}$ adjusted for CRRT). The patient died after 12 days of MER-VAB therapy, without a source control procedure (198). In vitro activity of MER-VAB was demonstrated against S. marcescens enzyme (SME)-positive isolates, with bactericidal activity against all four isolates tested (199).

(ii) In vitro activity against specific bacteria. MER-VAB has good in vitro activity against Burkholderia spp., including B. cepacia and B. gladioli, with most isolates demonstrating MICs below CLSI breakpoints for $P$. aeruginosa in one study. Among BLBLIs, MER-VAB and PIP-TAZ showed the greatest activity against Achromobacter spp. (105).

Emergence of resistance to MER-VAB during treatment. Assessing the development of the resistance during exposure to MER-VAB, an in vitro study by Sun et al. (200) on $18 \mathrm{KPC}$-producing $\mathrm{K}$. pneumoniae strains reported that after exposure to low drug concentrations, resistance was emerging due to an increase in the $b / a_{\mathrm{KPC}}$ copy number and ompK36 inactivation. Thereby, the authors concluded that the development of the resistance to MER-VAB could be prevented by the optimal drug concentrations (200). In the study described above by Shields et al. (196), 20 patients with CRE infections were treated with MER-VAB for $>48 \mathrm{~h}$. Within 90 days, microbiological failures (isolation of the same bacterial species following $\geq 7$ days of MER-VAB treatment) were noted in $35 \%(6 / 20)$ of cases. One-half of the recurrent isolates demonstrated a $\geq 8$-fold MERVAB MIC increase, and one was categorized as nonsusceptible to MER-VAB (MIC $=8 \mathrm{mg} /$ liter). Addressing the resistant isolate, it occurred in a patient with bacteremia due to a CAZ-AVI-resistant K. pneumoniae harboring KPC-31. Therapy with MER-VAB was initiated (MER-VAB, MIC of $0.12 \mathrm{mg} /$ liter), but after 12 days of treatment, the patient developed an abdominal wall abscess caused by $K$. pneumoniae nonsusceptible to MER-VAB (MIC = $8 \mathrm{mg} /$ liter). Whole-genome sequencing identified a new IS5 insertion in the ompK36 promoter of the recurrent isolate (196). However, data regarding emerging resistance after the exposure to MER-VAB are limited, and new resistance mechanisms may arise when MER-VAB becomes more widely used in the clinical practice.

Future clinical studies evaluating MER-VAB. No clinical trial is currently registered for MER-VAB at ClinicalTrials.gov. One trial was withdrawn by the sponsor in January 2019. This trial was registered in 2016 and was planned to compare MER-VAB versus PIP-TAZ for the treatment of HABP/VABP (201).

Data to support the use of MER-VAB in CRE infections are encouraging; however, they are limited to one small RCT and one small observational study.

\section{IMIPENEM-RELEBACTAM \\ Spectrum of Activity}

Imipenem-cilastatin is combined with relebactam mainly to restore the activity against KPC and other carbapenemase-producing Enterobacterales and $P$. aeruginosa isolates (202-204). The most common mechanisms of imipenem resistance in $P$. aeruginosa are decreased expression of OprD and overproduction of AmpC 
$\beta$-lactamases. Relebactam inhibits AmpC, thereby improving the activity of imipenem $(202,205)$. Several studies have reported that the combination of imipenem/cilastatinrelebactam (IMI-REL) is effective against bacteria carrying class $A$ and class $C$ $\beta$-lactamases, while the activity against $b / a_{\text {OXA-48 }}$-expressing carbapenem-resistant $E n$ terobacterales (CRE) is limited, and no activity against MBL (including IMP, VIM, and NDM)-producing isolates is demonstrated $(202,206-212)$. A recent study by Biagi et al. reported that IMI-REL had no activity against the carbapenem-resistant Serratia marcescens (199). The activity of relebactam against $\beta$-lactamases is summarized in Table 1.

A global surveillance program (SMART [Study for Monitoring Antimicrobial Resistance Trends]) including 21 clinical laboratories in the United States in 2015 reported that relebactam restored susceptibility to imipenem in $80.5 \%, 100 \%$, and $74.1 \%$ of imipenem-nonsusceptible $P$. aeruginosa, Enterobacter spp., and $K$. pneumoniae, respectively. Relebactam at fixed concentration of $4 \mathrm{mg} /$ liter in combination with doubling dilutions of imipenem was used and IMI-REL MIC interpreted according to CLSI imipenem breakpoints. Isolates producing OXA-48-type carbapenemases, metallo- $\beta$ lactamases (VIM), or the class A carbapenemase GES-20 were resistant to IMI-REL (213). The data from SMART surveillance between 2015 and 2017 assessing IMI-REL in vitro activity in isolates causing lower respiratory tract infections showed that $92.2 \%$ of $P$. aeruginosa, including $77.2 \%$ of imipenem-nonsusceptible and $79.6 \%$ of MDR isolates, were susceptible to IMI-REL (214). Similar results were reported in isolates from the intra-abdominal and urinary tract infections, where $96.7 \%$ and $96.4 \%$ of $P$. aeruginosa isolates, respectively, were susceptible to IMI-REL. When analyzing imipenem nonsusceptible and MDR P. aeruginosa, $85.0 \%$, and $87.3 \%$, respectively, were susceptible to the combination (215). It should be noted that the data are limited by the interpretation according to CLSI imipenem breakpoints, while currently, there are EUCAST-specific IMI-REL breakpoints (Table 2).

In a study by Livermore et al. (202), IMI-REL was active against AmpC- or ESBLproducing Enterobacterales with impermeability phenotypes (e.g., loss of porins). In the case of OprD-deficient $P$. aeruginosa strains, the MIC of imipenem dropped from 16 to $64 \mathrm{mg} /$ liter to 1 to $4 \mathrm{mg} /$ liter (202). In a study investigating bacterial isolates from 11 hospitals in New York (November 2013 to January 2014), the addition of relebactam decreased the MIC values of imipenem in $P$. aeruginosa isolates approximately 4 -fold (216). Nevertheless, relebactam did not improve the activity of imipenem against $A$. baumannii harboring bla $a_{\mathrm{OXA}-23 \text {-like }}$ or with overexpression of AmpC and/or OXA-51 $\beta$-lactamase $(213,216)$. In a recent study by Tooke et al., IMI-REL was reported as active against bacteria producing class $\mathrm{A} \beta$-lactamases, both chromosomal and plasmid-borne enzymes, i.e., ESBLs L2 and CTX-M-15 (211).

Evidence that IMI-REL inhibits Bla ${ }_{\text {Mab }}$ has been observed in a study evaluating in vitro activity of IMI-REL against Mycobacterium abscessus complex when relebactam reduced the $\mathrm{MIC}_{50}$ and $\mathrm{MIC}_{90}$ of imipenem from 16 to 8 and from 32 to 16, respectively (217). A study by Papp-Wallace et al. including 101 clinical isolates of KPC-producing Klebsiella pneumoniae, Klebsiella oxytoca, Klebsiella aerogenes (formerly Enterobacter aerogenes), Enterobacter cloacae, Citrobacter freundii, Citrobacter koseri, and Escherichia coli reported that all of the isolates were highly susceptible to IMI-REL (MICs $\leq$ $2 \mathrm{mg} / \mathrm{liter}$ ) (218). Similar results were documented in a study by Carpenter et al., where IMI-REL was the most potent agent combination tested against CRE: $\mathrm{MIC}_{50} / \mathrm{MIC}_{90}$ values of IMI-REL were $\leq 0.25 / 0.5 \mathrm{mg} /$ liter (219). Another study published in 2019 including $P$. aeruginosa clinical isolates showed IMI-REL susceptibility rates of $97.3 \%$, and MICs remained $\leq 2 \mathrm{mg} /$ liter, including for all isogenic PAO1 mutants and XDR clinical strains with mutations in OXA-10 or AmpC (220). The respective EUCAST-, FDA-, and CLSIapproved breakpoints for the interpretation of the susceptibility of particular pathogens to IMI-REL are summarized in Table 2. For antimicrobial susceptibility data of various pathogens to IMI-REL, see Table 4.

\section{Resistance Rates and Mechanisms}

The pathogens resistant to IMI-REL are mostly those producing class B metallo- $\beta$ - 
lactamases and some of the OXA class $D \beta$-lactamases. There is also an intrinsic resistance to carbapenems, where adding $\beta$-lactamase inhibitor does not increase the sensitivity, i.e., for Enterococcus faecium producing PBP5 $(175,179)$. Other resistance mechanisms are the decreased expression of OmpK porin proteins in K. pneumoniae, OmpC and OmpF in Enterobacter spp., and downregulation or mutations in OmpK36 porin $(179,209,216)$. Tested against $E$. cloacae, $P$. aeruginosa, and $C$. freundii strains, relebactam did not result in $A m p C$ induction for any of the strains (58).

Neither imipenem nor relebactam is a substrate of $P$. aeruginosa efflux (204). In a study by Haidar et al. published in 2017 , OmpK36 porin mutations in carbapenemresistant Enterobacterales were independently associated with higher MICs of IMI-REL (209). Assessing the development of the resistance during exposure, in a study by Noel et al. (221) using in vitro simulations of 7- or 14-day human exposures to IMI-REL on aerobic Gram-negative bacilli, there were no changes in MICs after the first 7 days of exposure. Meanwhile, in P. aeruginosa, there was increase in MIC and regrowth in the 14-day model using monotherapy with IMI-REL. The addition of amikacin increased the clearance of $P$. aeruginosa and prevented the development of resistance. The authors concluded that IMI-REL was effective and there was no emergence of resistance in Enterobacterales. The drug was active against $P$. aeruginosa, but in a 14-day model, regrowth and resistance were detected and were prevented with the addition of amikacin (221).

\section{PK-PD Characteristics}

Like other BLBLIs, imipenem and relebactam exhibit very similar plasma protein binding, half-lives, $V_{\text {ss }}$ values (as summarized in Table 5), and routes of elimination (222). Consistent with other $\beta$-lactams and $\beta$-lactamase inhibitors, both drugs are predominantly excreted unchanged in urine. Wu et al. modeled data from checkerboard assays to describe a dynamic PK-PD index (time above dynamic MIC $\left.\left[T_{>\text {MiCdynamic }}\right]\right)$ that simultaneously accounted for the activities of both imipenem and relebactam (223). Unlike traditional MIC assessments, the dynamic MIC describes the MIC of imipenem as a function dependent on various relebactam concentrations encountered over a typical dosing interval. Hollow-fiber experiments were used to derive a target $\% f T_{>\text {MIC }}$ dynamic predictive of the efficacy of the combination. FDA approval for the current indications ( $\mathrm{ClAl}$ and CUTI) relied on assessment of target

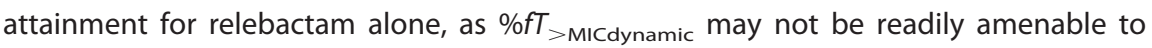
traditional PTA analyses. As with other inhibitors, the PK-PD index of relebactam was characterized using a fixed imipenem exposure (15). A target $f A U C_{0-24} / \mathrm{MIC}$ of 14.4 for 1 - $\log _{10}$ reduction in baseline inoculum (for $\beta$-lactam-expressing isolates) obtained from murine thigh infection experiments was evaluated in PTA analysis and used to support the approved dose of $0.25 \mathrm{~g}$ relebactam with $1 \mathrm{~g}$ imipenem/cilastatin (15).

\section{Clinical Data}

Approval date and indications. IMI-REL is FDA and EMA approved. It was first approved by the FDA in July 2019 for use in adults with CUTI and CIAI. The dosage

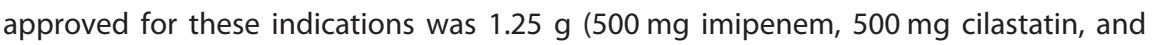
$250 \mathrm{mg}$ relebactam infused over 30 min every $6 \mathrm{~h}$ ). It has not yet been approved for use in pediatric patients $(222,224)$.

Randomized controlled trials evaluating IMI-REL for any infection. Three RCTS have been published so far evaluating IMI-REL for various infections. Sims et al. (225), in a phase 2 RCT, compared two different doses of IMI-REL versus imipenem alone for cUTI. Study arm dosing included high-dose relebactam (imipenem/cilastatin $500 \mathrm{mg}$ plus relebactam $250 \mathrm{mg}$ ) versus low-dose relebactam (imipenem/cilastatin $500 \mathrm{mg}$ plus relebactam $125 \mathrm{mg}$ ) versus imipenem/cilastatin $500 \mathrm{mg}$ alone (plus placebo). All drugs were administered over $30 \mathrm{~min}$ intravenously every $6 \mathrm{~h}$. Overall, 302 patients were randomized. Among 230 patients who had a baseline pathogen, most common was $E$. coli $(62 \%) ; 50.2 \%$ had multidrug-resistant bacteria, and $11 \%$ of all pathogens were imipenem nonsusceptible at baseline. No cases of mortality were documented and no 
differences between study arms were demonstrated for either clinical or microbiological response at EOT, early follow-up at 5 to 9 days after completion of treatment, or long-term follow-up, 28 to 42 days after completion of all study therapy. No differences in microbiological response were demonstrated between study arms for the subgroups of bacteremia, elderly patients (age, $\geq 65$ years), or patients with abnormal renal function. AEs were reported in the investigational arms in $28 \%$ to $29 \%$ of patients, most commonly, gastrointestinal side effects, headaches, hypertension, and liver function test abnormalities. SAEs and AEs requiring drug discontinuation were not common and without a significant difference between IMI-REL arms and imipenem (225) (Table 14).

Lucasti et al. (226) conducted a similar phase 2 dose-ranging study in patients with CIAl. Three hundred fifty-one patients were enrolled, and only $21 \%$ were elderly (age, $\geq 65$ years), $4 \%$ had bacteremia, and $4 \%$ had an APACHE II score of $>15$. Among 250 patients who had a baseline pathogen, most common was E. coli (65\%), and $13 \%$ of patients had an imipenem-nonsusceptible pathogen at baseline. No differences between study arms were demonstrated for either the clinical or microbiological response at EOT, early follow-up at 5 to 9 days after completion of treatment, or long-term follow-up, 28 to 42 days after completion of all study therapy. Any AEs were reported in the investigational arms in $47 \%$ to $48 \%$ of patients, most commonly, gastrointestinal side effects and liver function test abnormalities. No significant difference between IMI-REL arms and imipenem was demonstrated in rates of SAEs, AEs requiring drug discontinuation, and mortality, though all were more common in the low-dose IMI-REL arm (226) (Table 14).

The RESTORE-IMI 1 was a phase 3 RCT, comparing IMI-REL alone versus imipenem combined with colistin for imipenem-nonsusceptible infections (227). The dosage of IMI-REL was 500 and $250 \mathrm{mg}$ every $6 \mathrm{~h}$; the dosages of imipenem and colistin were $500 \mathrm{mg}$ every $6 \mathrm{~h}$ and $4.5 \mathrm{MU}$ every $12 \mathrm{~h}$, respectively. The infections included were HABP/NABP, cIAI, and CUTI. Overall, 31 patients were included in the IMI-REL arm and 16 in the comparator arm (imipenem plus colistin). Including both arms, overall, 31 patients had a baseline pathogen, 24 patients had $P$. aeruginosa, and 7 had various Enterobacterales, 6 of them KPC or OXA-48 producing. Of the 31 microbiologically evaluable patients, 11 (35.5\%) were 65 years and older, more commonly in the comparator arm (50\% versus 28\%), 9 had an APACHE II score of $>15$ (7 IMI-REL and 2 comparator), and 7 had $\mathrm{CrCL}$ of $<60 \mathrm{ml} / \mathrm{min}$ (4 IMI-REL and 3 comparator). The primary outcome was overall response, defined differently for each type of infection (Table 14). There was no significant difference between study arms for the primary outcome (71.4\% versus $70 \%)$. Twenty-eight-day all-cause mortality was lower with IMI-REL (2/21 [9.5\%] versus $3 / 10$ [30\%]), and clinical response at 28 days was significantly higher with IMI-REL (71\% versus $40 \%$ ) (Table 14). AEs occurred in $71.0 \%$ of patients treated with IMI-REL compared to $81 \%$ with comparator. Most common AEs were pyrexia, nausea, decreased creatinine renal clearance, and abnormal liver function tests, all of which were more common in the comparator arm. Specifically, nephrotoxicity was significantly more common in the comparator arm (10\% versus $56 \%$ ). SAEs and AEs requiring drug discontinuation were more common with the comparator (227) (Table 14).

Other clinical studies for IMI-REL. (i) IMI-REL in pediatric populations. Currently, a phase 2/3 RCT comparing IMI-REL versus active control for Gram-negative infections in pediatric population is ongoing (228).

(ii) Other clinical studies. NCT03293485 is an ongoing, interventional single-arm study evaluating IMI-REL for the treatment of CIAI and CUTI in Japanese participants. Results from 83 included participants were posted on ClinicalTrials.gov, showing an overall low mortality of 1 of 83 patients, clinical response of $82 \%$ (28/34) for clAl, and 100\% microbiological response for CUTI (229) (Table 14).

Future clinical studies evaluating IMI-REL. Two phase 3 RCTs evaluating IMI-REL versus PIP-TAZ for HABP/VABP in adults are ongoing. One is currently recruiting patients, aiming for a sample size of 270 patients (230), and the other has been completed and awaiting results for 537 enrolled participants (231). 


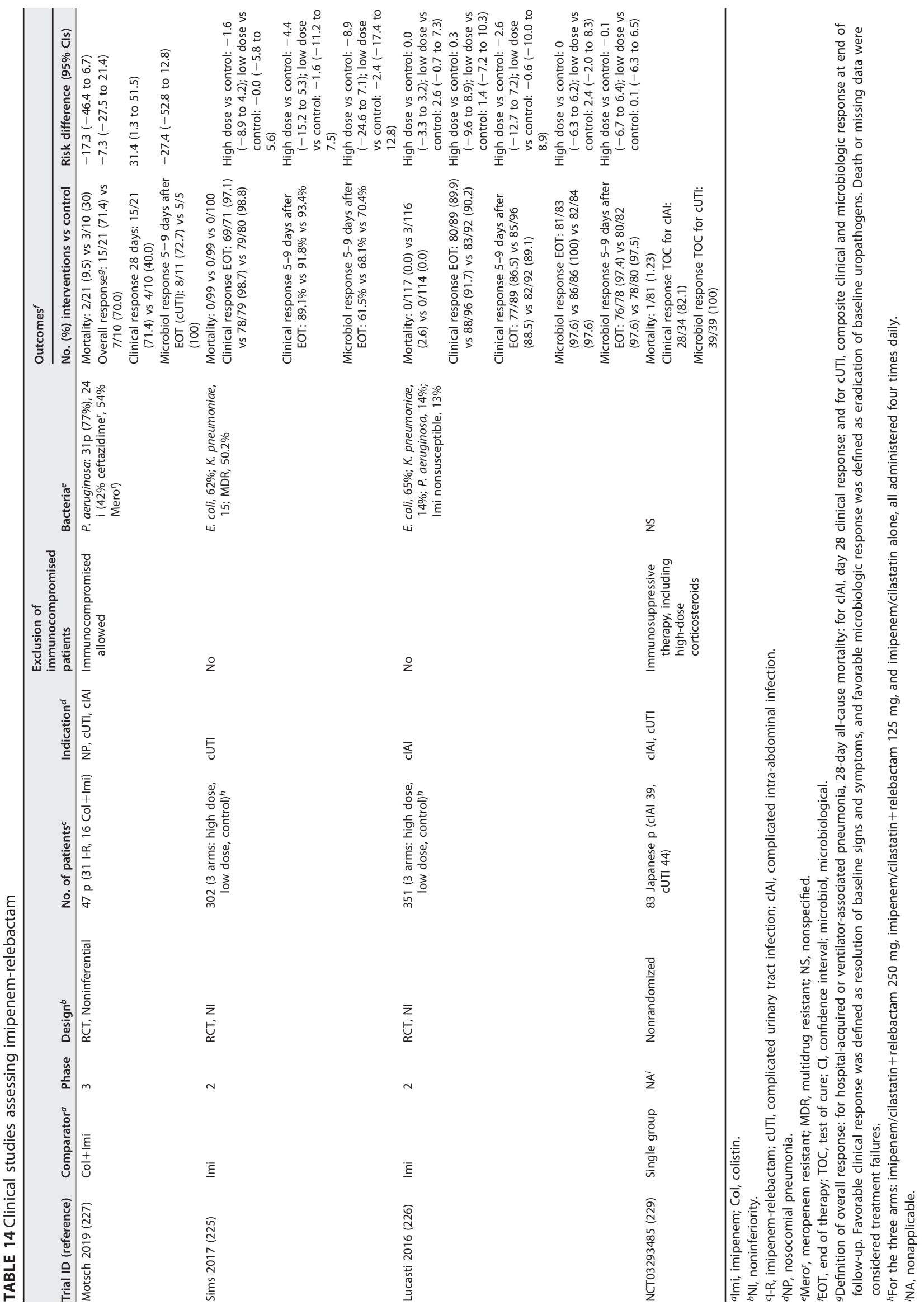


No future RCTs to evaluate the use of IMI-REL for MDR infections are registered, and further data are needed.

\section{BLBLIS IN DEVELOPMENT PROCESS}

The BLBLIs in development are summarized in Table 15.

\section{$\boldsymbol{\beta}$-Lactam-Sulfone $\boldsymbol{\beta}$-Lactamase Inhibitor Combinations}

Cefepime-tazobactam. Cefepime-tazobactam is licensed for clinical use by the Drugs Controller General of India. The preparations used in India are 8:1 cefepimetazobactam (maximum 1,000 mg/250 mg per vial). A 1:1 preparation has been tested in a phase 1 study and was shown to be well tolerated at a dose of up to $2 \mathrm{~g}$ and $2 \mathrm{~g}$ intravenously every $8 \mathrm{~h}$ for up to 7 days. The spectrum of activity includes Enterobacterales that are $\mathrm{AmpC}, \mathrm{ESBL}, \mathrm{K} 1$, or OXA-48 $\beta$-lactamase producing, with coverage similar to that of meropenem for these pathogens. KPC- and NDM-producing Enterobacterales are mostly resistant, though susceptibility of $\sim 75 \%$ of isolates with VIM has been demonstrated. The latter was explained by the weak activity of VIM against cefepime specifically, supported by activity of tazobactam against coproduction of ESBLs. For $P$. aeruginosa and other nonfermenters, the addition of tazobactam results in minimal changes in MIC, and the activity of cefepime-tazobactam is comparable to that of other antipseudomonal $\beta$-lactams (meropenem, piperacillin-tazobactam, and ceftazidime) $(232,233)$.

Clinical experience is reported only from India. In a retrospective study from India, 154 patients were treated with cefepime-tazobactam, mainly for pneumonia (31\%) or UTI (23\%) caused by ESBL-producing Klebsiella pneumoniae or E. coli (234). Twenty-nine percent of patients were admitted to an ICU. Clinical improvement was documented in 142 patients (92.2\%), and 2 patients died. No adverse events were reported other than diarrhea in 6 patients (234). Ghafur et al. (235) included in a retrospective study 32 patients, adults and children, who were treated with cefepime-tazobactam in an Indian hospital with endemic occurrence of ESBL producers. The effectiveness analysis was limited to 15 patients who received the drug for a clear-source (12 respiratory infections) monomicrobial infection (5 P. aeruginosa, 3 Acinetobacter spp., and 7 Enterobacterales) treated with cefepime-tazobactam alone. All 15 patients improved clinically and were discharged from the hospital. The safety analysis included all 32 patients, and none of them experienced a serious adverse event (235).

An interventional RCT comparing cefepime-tazobactam ( $2 \mathrm{~g}$ and $2 \mathrm{~g}$ ) versus meropenem for CUTI is ongoing (236).

Cefepime-enmetazobactam (AAl101). Enmetazobactam is a penicillanic acid sulfone $\beta$-lactamase inhibitor with no intrinsic activity against Gram-negative bacteria. It has a similar structure to that of tazobactam, with a difference in a single methyl group that gives the drug a net neutral charge, promoting bacterial wall penetration. This structural difference enables enmetazobactam to form more hydrogen bonds in the active site of class $A$-lactamases than tazobactam, resulting in possibly delayed turnover of enmetazobactam (237). The combination was shown to be as effective as carbapenems against ESBLs in vitro. In addition, cefepime itself is relatively stable against hydrolysis by AmpC and OXA $\beta$-lactamases; thus, the combination is active against class $A, C$, and $D \beta$-lactamases (237). Broth microdilution and disk diffusion quality control ranges for cefepime-enmetazobactam were recently set by CLSI (238). Testing the drug against a panel of 1,696 Enterobacterales isolates, the addition of enmetazobactam to cefepime lowered the $\mathrm{MIC}_{90}$ by seven doubling dilutions (from 32 to $0.25 \mu \mathrm{g} / \mathrm{ml}$ ) compared to that of cefepime alone. The effect was substantial for ESBLs (all but one turned from resistant to susceptible) but limited for KPC- and VIMproducing Enterobacterales. This was not shown for $P$. aeruginosa, for which enmetazobactam did not enhance the potency of cefepime. Using a cefepime breakpoint of $8 \mathrm{mg} / \mathrm{liter}$, the addition of enmetazobactam to cefepime rendered $82.8 \%$ of isolates susceptible (239).

An ongoing phase 3, randomized, controlled, double-blind noninferiority trial is 


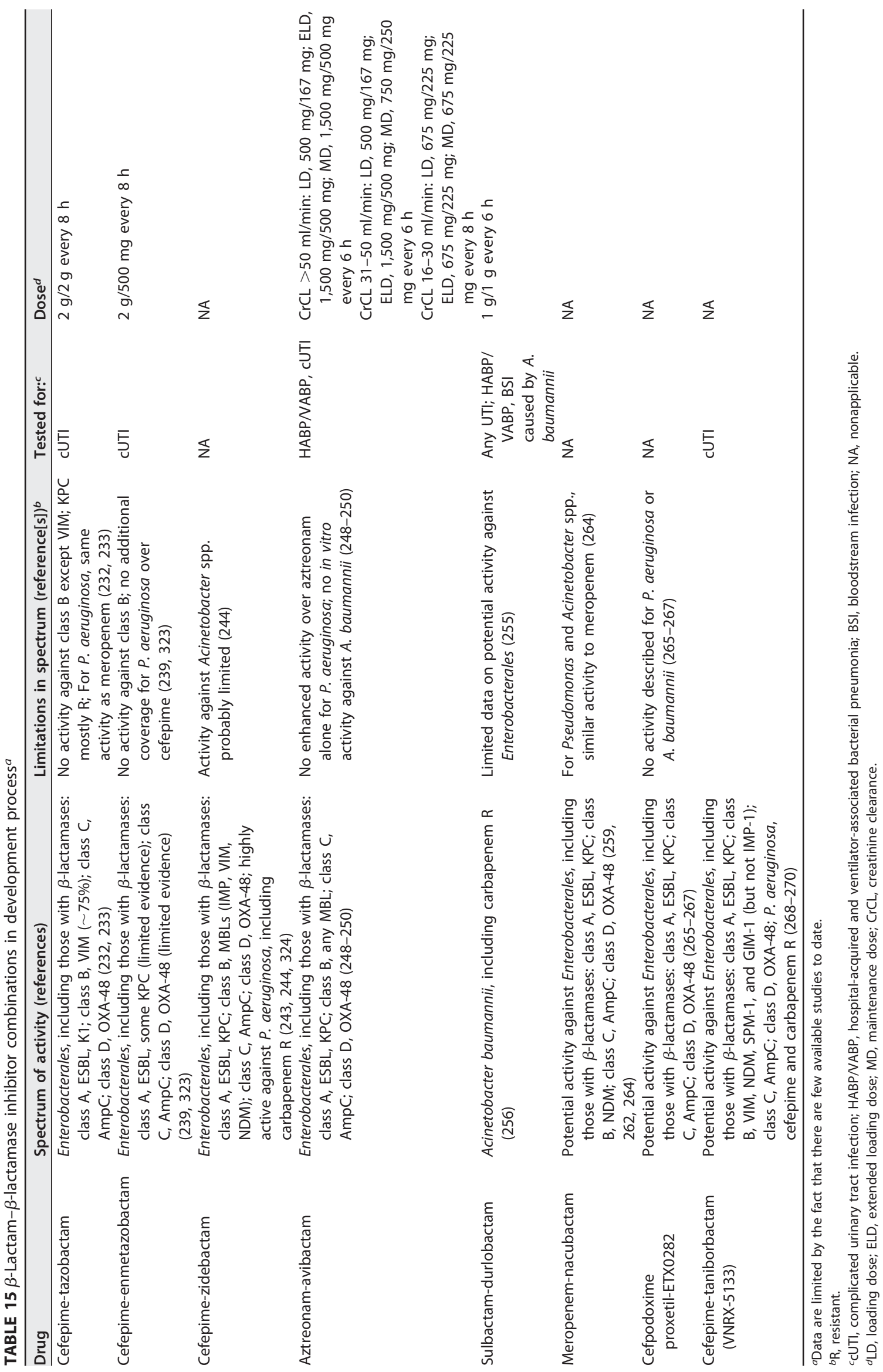


currently recruiting adult patients with cUTI for treatment with cefepime 2 g/AAl101 $500 \mathrm{mg}$ every $8 \mathrm{~h}$ (q8h) versus piperacillin $4 \mathrm{~g} /$ tazobactam $500 \mathrm{mg}$ q8h. The primary outcome is overall treatment success at test of cure, and the trial is planned to include 1,040 patients (240).

\section{$\boldsymbol{\beta}$-Lactam-Diazabicyclooctane $\boldsymbol{\beta}$-Lactamase Inhibitor Combinations}

Diazabicyclooctanes (DBOs) are a class of $\beta$-lactamase inhibitors that includes older DBOs (avibactam and relebactam) and newer DBOs (zidebactam, nacubactam, durlobactam, and ETX0282). The latter ones possess the ability to inhibit PBPs and are thus considered $\beta$-lactam enhancers, acting synergistically with the partner $\beta$-lactam on different PBPs in addition to the $\beta$-lactamase inhibitor activity (241).

Cefepime-zidebactam (WCK 5107). Zidebactam is a non- $\beta$-lactam that inhibits class A and metallo- $\beta$ lactamases (MBLs). In addition, it also inhibits PBP2. The four approved new BLBLIs described above do not provide in vitro activity against MBL. Cefepimezidebactam, not yet approved for clinical use, has demonstrated promising in vitro activity against MBL-positive pathogens. Recent studies have demonstrated $90 \%$ to $100 \%$ susceptibility to cefepime-zidebactam among $35 \mathrm{MBL}$-positive CPE strains, including coproducers of serine $\beta$-lactamases. Cefepime-zidebactam was also demonstrated to have in vitro activity against $P$. aeruginosa with AmpC overexpression and MBLs, with activity against $78 \%$ to $98 \%$ of meropenem-nonsusceptible strains and $97 \%$ of ceftazidime-nonsusceptible strains. It has also documented moderate activity against OXA-23/24/58-producing Acinetobacter baumannii, with 4-fold higher activity than cefepime or ceftazidime for this pathogen. In one study, $85 \%$ of isolates and $34 \%$ of carbapenem-resistant isolates had MICs below the susceptible-dose-dependent breakpoint of cefepime (242-244). Breakpoints and recommended testing methods are still pending, and no clinical trial is yet registered for this drug (245).

Kaushik et al. (246) demonstrated that addition of zidebactam to cefepime reduced the latter compound's MIC $_{50}$ to Mycobacterium abscessus 2-fold, from 32 to $16 \mathrm{mg} / \mathrm{liter}$. These results are limited by the fact that there are no established breakpoints for cefepime against Mycobacterium abscessus, and the results were determined considering CLSI cefepime breakpoints for $P$. aeruginosa (246).

Aztreonam-avibactam. The combination of the monobactam aztreonam together with avibactam has been shown as having in vitro activity against Enterobacterales with class $\mathrm{B}, \mathrm{A}, \mathrm{C}$, and some $\mathrm{D} \beta$-lactamases. Aztreonam is stable for hydrolysis by MBLs but not by most serine $\beta$-lactamases. The addition of avibactam has the potential to inhibit other classes and provide coverage for MBL-producing isolates, which usually coproduce serine $\beta$-lactamases. Kazmierczak et al. tested 333 Enterobacterales isolates carrying $b / a_{\mathrm{OXA}-48}$ and $b / a_{\mathrm{OXA}-48 \text {-like }}$ genes and found $>99.6 \%$ susceptibility to aztreonamavibactam (247). Sader et al. reported aztreonam-avibactam to be highly active against $>10,000$ Enterobacterales isolates, including $\sim 100 \mathrm{KPC}$ isolates, $\sim 60$ OXA-48-like producers, and $\sim 60 \mathrm{MBL}$-positive isolates (248). An additional large study demonstrated similar results for $>50,000$ Enterobacterales isolates tested, with potent activity of aztreonam-avibactam against these isolates, including meropenem-nonsusceptible and $\mathrm{MBL}$-positive isolates. In this study, the in vitro activity of aztreonam-avibactam against $P$. aeruginosa was less potent $\left(\mathrm{MIC}_{90}, 32 \mathrm{mg} / \mathrm{liter}\right.$ ), with avibactam addition not improving the activity of aztreonam. The authors concluded that resistance was probably caused, at least partially, by mechanisms other than $\beta$-lactamases (249). Neither aztreonam alone nor the combination of aztreonam-avibactam has in vitro activity against A. baumannii (250).

First posted in 2017, a phase 3 randomized controlled trial comparing aztreonamavibactam with or without metronidazole versus meropenem with or without colistin for the treatment of HABP/VABP and CIAI is ongoing. Clinical cure at 28 days is the primary outcome in this trial, which plans to include 375 patients (251).

A phase $2 a$, prospective single-arm study recently evaluated 34 adults treated with aztreonam-avibactam plus metronidazole for clAl. Twenty-three had microbiologically documented infection, and none of them had either ESBL- or MBL-positive isolates. The 
PK-PD assessment demonstrated that mean exposures of the drug components were consistent with those predicted to achieve PTA in $>90 \%$ of patients. Twenty-three patients (67\%) had any $A E$, most commonly, liver function abnormalities and diarrhea. Clinical cure rate at day 25 was overall 20/34 (58.8\%). Dosage data from this study supported the regimen selected for the phase 3 trial (loading dose of 500/167 mg administered over $30 \mathrm{~min}$, followed by $1,500 / 500 \mathrm{mg}$ administered over $3 \mathrm{~h}$ every $6 \mathrm{~h}$ regimen [in patients with $\mathrm{CrCL}>50 \mathrm{ml} / \mathrm{min}$ ]) (252).

Decreased susceptibility to aztreonam-avibactam has been reported to be due to PBP3 alteration by a 4-amino-acid insertion in E. coli strains (253).

The combination of aztreonam with avibactam was also tested for in vitro activity against Stenotrophomonas maltophilia, using CLSI breakpoints for $P$. aeruginosa as reference. Aztreonam-avibactam reduced the MIC compared to that for aztreonam alone from $>128$ to $8 \mathrm{mg} /$ liter for most isolates tested in one study (254).

Sulbactam-durlobactam (ETX2514). Sulbactam is a $\beta$-lactam with intrinsic activity against $A$. baumannii (through affinity to PBP1 and PBP3) and is also known as a $\beta$-lactamase inhibitor with activity against class $A \beta$-lactamases. Its activity as a single agent against $A$. baumannii is limited due to its hydrolysis by various $\beta$-lactamases produced by the bacteria, in particular, those of class D. Durlobactam (ETX2514) is a $\beta$-lactamase inhibitor that inhibits class $A, C$, and $D \beta$-lactamases. It also presents $\beta$-lactam properties, inhibiting PBP2 and thus having activity against some Enterobacterales (255). The antibacterial activity of the combination sulbactam-durlobactam was recently tested against 1,722 clinical isolates of Acinetobacter sp., predominantly, $A$. baumannii. Among these isolates, $\sim 50 \%$ were carbapenem resistant, and durlobactam added to sulbactam lowered the $\mathrm{MIC}_{90}$ by 32 -fold compared to that of sulbactam alone, from $64 \mathrm{mg} /$ liter to $2 \mathrm{mg} /$ liter. This level of activity was consistent among susceptible and resistant strains. Among strains with a sulbactam-durlobactam MIC of $>4 \mathrm{mg} / \mathrm{liter}$, either NDM-1 or PBP3 alteration was demonstrated (256).

Results from a double-blind, randomized controlled trial comparing sulbactamdurlobactam plus imipenem versus placebo plus imipenem for UTI were published recently (257). Fifty-three patients were randomized to receive $1 \mathrm{~g}$ durlobactam $/ 1 \mathrm{~g}$ sulbactam infused over $3 \mathrm{~h}$ q6h plus $500 \mathrm{mg}$ imipenem q6h, and 27 patients were randomized to receive same dose of imipenem with placebo. Overall success (clinical cure and microbiologic eradication) at 21 days was achieved in $76.6 \%$ (36/47) in the intervention arm versus $81.0 \%(17 / 21)$ in the control arm. None of the patients died during the study, and no SAEs were reported. The most common adverse events with the drug were headache, nausea, and diarrhea. The PK profile was consistent with that demonstrated in health volunteers (257).

A phase 3 evaluating sulbactam-durlobactam plus imipenem versus colistin plus imipenem for HABP/VABP and bacteremia caused by $A$. baumannii is currently recruiting patients (258).

Meropenem-nacubactam (FPI-1465). Nacubactam is a non- $\beta$-lactam $\beta$-lactamase inhibitor with in vitro activity against class $A, C$, and some class $D \beta$-lactamases. In addition, it has affinity to PBP2 and hence acts as an enhancer, conferring activity against MBLproducing Enterobacterales (259). The combination meropenem-nacubactam has been tested against seven meropenem-resistant $P$. aeruginosa clinical isolates in a neutropenic murine lung infection model, showing substantial reductions of bacterial burden (260). Similarly, enhanced activity of the combination was demonstrated in animal models against class A serine carbapenemase-producing Enterobacterales isolates and other MDR Enterobacterales, including NDM-, KPC-, OXA-, CTX-M-, SHV-, and TEM-producing isolates (261, 262). Nacubactam, with its intrinsic PBP2 activity, may be a potential agent for strains with ceftazidime-avibactam resistance due to mutation in the $\Omega$-loop (263). In a large in vitro study, meropenem-nacubactam inhibited $>99.5 \%$ of 3,306 Enterobacterales isolates tested (264). Among 117 meropenem-nonsusceptible or MDR Enterobacterales, 87.2\%, 92.3\%, and $96.6 \%$ were inhibited at $\leq 2, \leq 4$, and $\leq 8 \mathrm{mg} /$ liter, respectively. In addition, for 33 of 37 ceftazidime-avibactam-resistant Enterobacterales, meropenem-nacubactam displayed an MIC of $\leq 8 \mathrm{mg} /$ liter. In the same study, for 960 Pseudomonas spp. and 429 Acinetobacter 
spp., the combination had similar activity to meropenem alone (264). In an evaluation of in vitro activities of meropenem-nacubactam against clinical isolates of Mycobacterium abscessus complex, addition of nacubactam lowered the $\mathrm{MIC}_{50}$ of meropenem from 16 to 2 mg/liter in Middlebrook $7 \mathrm{H} 9$ medium (246).

No clinical trials aiming to assess the efficacy of meropenem-nacubactam are yet registered at ClinicalTrials.gov.

Cefpodoxime proxetil-ETX0282 (active compound ETX1317). Cefpodoxime proxetilETX0282 is the only orally administered $\beta$-lactam $\beta$-lactamase inhibitor under clinical development to date. ETX0282 is a prodrug of ETX1317, a $\beta$-lactamase inhibitor with activity against serine $\beta$-lactamases of class A, C, and a selection of class $D$. In addition, it has intrinsic antibacterial activity against some species. ETX1317 is combined with a $\beta$-lactam prodrug, cefpodoxime proxetil, hydrolyzed in vivo to cefpodoxime. It was found that cefpodoxime-ETX1317 in a 1:2 fixed ratio has the most potent activity and probably the best correlation to in vivo efficacy (265). This combination has been tested in vivo on 1,875 Enterobacterales urinary clinical isolates. In this study, addition of ETX1317 lowered cefpodoxime $\mathrm{MIC}_{50}$ and $\mathrm{MIC}_{90}$ from of 0.5 and $>16 \mathrm{mg} /$ liter to 0.06 and $0.12 \mathrm{mg} /$ liter, regardless of the resistance phenotypes and type of bacteria (265). It has also been tested in vitro on 30 isolates of Enterobacterales with various resistance genes and phenotypic resistance to carbapenems or colistin and showed potent antibacterial activity $(265,266)$. In addition, isolates of $\mathrm{KPC} 3$ with resistance mutations to ceftazidime-avibactam (V240G, D179Y, and D179Y/T243M) were inhibited by the combination. MICs for cefpodoxime proxetil-ETX0282 were 0.12 to $0.25 \mathrm{mg} / \mathrm{liter}$ both for wild-type and for mutant KPC-3 isolates (267).

\section{$\boldsymbol{\beta}$-Lactam-Boronate $\boldsymbol{\beta}$-Lactamase Inhibitor Combinations}

Cefepime-taniborbactam (VNRX-5133). Taniborbactam is a boronic-acid-containing $\beta$-lactamase inhibitor that inhibits class $A, C, D$, and even class $B \quad \beta$-lactamases, including VIM, NDM, SPM-1, and GIM-1 (but not IMP). The inhibition of serine $\beta$-lactamases occurs while the drug covalently binds to the site serine residue, producing enzyme-mediated hydrolysis. The inhibition of metallo- $\beta$-lactamases involves interaction of the boron moiety with the active zinc site, inducing narrowing of the active site cleft (268). The combination cefepime-taniborbactam has been demonstrated to provide potent activity against strains with an elevated MIC to ceftazidime-avibactam (producing 171KPC-3 $\Omega$-loop variants D179Y, V240G, A177E/D179Y and D179Y/T243M). It was recently demonstrated to have potent in vitro activity against Enterobacterales and $P$. aeruginosa. Among 817 P. aeruginosa isolates nonsusceptible to cefepime, meropenem, or both, $70 \%$ were inhibited at the susceptible breakpoint of $8 \mathrm{mg} / \mathrm{liter}$, and overall, $85 \%$ were inhibited at $16 \mathrm{mg} /$ liter (268-270). A phase 3, randomized, double-blind noninferiority study is currently recruiting patients to evaluate cefepimetaniborbactam versus meropenem for the treatment of cUTI in adults (271). The primary outcome is a composite of microbiological eradication and symptomatic clinical success at test of cure.

QPX7728. QPX7728 is an additional boronic-acid-containing $\beta$-lactamase inhibitor, with ability to inhibit class A ESBLs and carbapenemases (KPC) in class B (NDM, VIM, IMP), class C, and class D (OXA-48 in Enterobacterales and OXA-23 in A. baumannii). This compound was tested against carbapenem-resistant $A$. baumannii and $P$. aeruginosa isolates, combined with several $\beta$-lactams. Meropenem, ceftolozane, piperacillin, and cefepime all demonstrated increased potency with the addition of QPX7728 (272). Similarly, combinations of several $\beta$-lactams with QPX7728 restored their activity against CRE producing either KPC, OXA-48-like, or metallo- $\beta$-lactamases (273). It was also tested against CAZ-AVI-resistant KPC-producing isolates, with retained activity attributed to their different binding sites (274). The potency of the drug is also retained in $P$. aeruginosa with inactivation of the OprD porin, and it is also minimally affected by efflux pumps. There is currently no fixed combination of this compound with a 
TABLE 16 Different resistance mechanisms among Enterobacterales and $P$. aeruginosa against four approved new BLBLIs ${ }^{a}$

\begin{tabular}{ll}
\hline Drug $^{b}$ & Resistance mechanisms \\
\hline CAZ-AVI & Class B MBLs \\
& Hyperexpression of efflux pumps \\
& Porin alterations \\
& Increased expression of the bla $a_{\text {KPC }}$ gene or mutations on $\Omega$-loop of KPC enzymes \\
& Mutations in PBPs (rare)
\end{tabular}

TOL-TAZ Class A $\beta$-lactamases (some of ESBLs, mainly K. pneumoniae, most of KPCs)

Class B MBLs

Hyperproduction of AmpC (not in P. aeruginosa)

Class D carbapenemases (OXA-48-like)

MER-VAB Class B MBLs

Class D carbapenemases (OXA-48-like)

Porin alterations

Hyperexpression of efflux pumps

IMI-REL Class B MBLs

Class D carbapenemases (OXA-48-like)

Specific class A carbapenemases (e.g., GES)

Hyperexpression of KPC

Porin alterations

asee references 325 to 329 .

${ }^{b}$ CAZ-AVI, ceftazidime-avibactam; TOL-TAZ, ceftolozane-tazobactam; MER-VAB, meropenem-vaborbactam;

IMI-REL, imipenem-relebactam.

$\beta$-lactam, and it has been suggested as a "stand-alone" drug, though the latter approach is still debated (275).

\section{INSIGHTS FROM THE EXPERTS}

Novel BLBLIs enable new options of treatment for carbapenem-resistant Enterobacterales, $P$. aeruginosa, $A$. baumannii, and other bacteria with limited treatment options, including mycobacteria. They also constitute a carbapenem-sparing option for the treatment of common infections, including those caused by ESBL/AmpC-producing Enterobacterales and non-carbapenem-resistant $P$. aeruginosa. Nevertheless, currently, this alternative is limited by the high cost of novel BLBLIs.

Current use of the four approved BLBLIs should probably be as definitive therapy for isolates resistant to other treatment options. CAZ-AVI could be an option for most resistant Enterobacterales, including ESBL-, KPC-, AmpC-, and OXA-48-producing isolates. It may also be used for CAZ-AVI-susceptible $P$. aeruginosa carbapenem-resistant isolates. TOL-TAZ and, to some extent, ESBLs, could be used mainly for carbapenemresistant $P$. aeruginosa. MER-VAB and IMI-REL, similarly to CAZ-AVI, could be used for various resistant Enterobacterales, however, with no coverage for class $D \beta$-lactamaseproducing pathogens. For $P$. aeruginosa, the activity is of MER-VAB is similar to that of meropenem alone. Relebactam restores imipenem's activity in $\sim 80 \%$ of resistant $P$. aeruginosa strains. None of these drugs is active against MBL-producing bacteria or carbapenemase-producing $A$. baumannii. For resistance mechanisms for each of the four drugs, see Table 16.

Most clinical data on new BLBls come from two types of studies.

1. Randomized controlled trials, with a noninferiority design, conducted mostly for CUTI or CIAI in patients with no immunosuppression and nonsevere infection and including mostly nonresistant bacteria. These trials have limited external validity for use of these drugs to treat MDR pneumonia or bacteremia. Exceptions are the TANGO II trial (193), evaluating MER-VAB exclusively in CRE infections, and the RESTORE-IMI 1 (227), evaluating IMI-REL for imipenem-nonsusceptible bacterial infections. Postmarketing trials including patients with carbapenem-resistant bacterial infections are not listed at ClinicalTrials.gov. One trial in an immuno- 
compromised population is registered for TOL-TAZ (for neutropenic fever); another trial is registered for treating ESBL and AmpC producers.

2. Real-life small retrospective studies focusing on treating CRE infections with CAZ-AVI and MER-VAB, with few data addressing MDR/XDR $P$. aeruginosa for these drugs, and on treating MDR/XDR $P$. aeruginosa with TOL-TAZ, with limited data on Enterobacterales for this drug.

Future randomized controlled trials would best define the role of different novel BLBLIs in the treatment of carbapenem-resistant and other MDR infections. Since such studies are difficult to conduct and none are currently registered at ClinicalTrials.gov, observational data are needed to further define efficacy and the magnitude of resistance emergence.

\section{ACKNOWLEDGMENTS}

V.H.T. is supported by the National Institutes of Health (R01Al140287-02).

This study had no funding.

Contributions based on work were completed while employed at the University of Houston College of Pharmacy. The opinions expressed in this article are those of the authors and should not be construed to represent the Food and Drug Administration's views or policies.

\section{REFERENCES}

1. Tacconelli E, Carrara E, Savoldi A, Harbarth S, Mendelson M, Monnet DL, Pulcini C, Kahlmeter G, Kluytmans J, Carmeli Y, Ouellette M, Outterson K, Patel J, Cavaleri M, Cox EM, Houchens CR, Grayson ML, Hansen P, Singh N, Theuretzbacher U, Magrini N, WHO Pathogens Priority List Working Group. 2018. Discovery, research, and development of new antibiotics: the WHO priority list of antibiotic-resistant bacteria and tuberculosis. Lancet Infect Dis 18:318-327. https://doi.org/10.1016/ S1473-3099(17)30753-3.

2. Boucher HW, Talbot GH, Benjamin DK, Bradley J, Guidos RJ, Jones RN, Murray BE, Bonomo RA, Gilbert D, for the Infectious Diseases Society of America. 2013. $10 \times$ '20 progress-development of new drugs active against Gram-negative bacilli: an update from the Infectious Diseases Society of America. Clin Infect Dis 56:1685-1694. https://doi.org/10 $.1093 / \mathrm{cid} / \mathrm{cit} 152$.

3. Bush K, Macielag MJ. 2010. New $\beta$-lactam antibiotics and $\beta$-lactamase inhibitors. Expert Opin Ther Pat 20:1277-1293. https://doi.org/10.1517/ 13543776.2010.515588.

4. FDA. 2014. Ceftolozane-tazobactam. Center for Drug Evaluation and Research. Approval package for application number 2068290rig1s000. FDA, Silver Spring, MD.

5. FDA. 2015. Ceftazidime-avibactam. Center for Drug Evaluation and Research. Approval package for application number 2064940rig1s000. AVYCAZ injection. FDA, Silver Spring, MD.

6. Pfaller MA, Barry AL, Fuchs PC, Gerlach EH, Hardy DJ, McLaughlin JC. 1993. Comparison of fixed concentration and fixed ratio options for dilution susceptibility testing of gram-negative bacilli to ampicillin and ampicillin/sulbactam. Eur J Clin Microbiol Infect Dis 12:356-362. https://doi.org/10.1007/BF01964434.

7. Onufrak NJ, Forrest A, Gonzalez D. 2016. Pharmacokinetic and pharmacodynamic principles of anti-infective dosing. Clin Ther 38: 1930-1947. https://doi.org/10.1016/j.clinthera.2016.06.015.

8. Tam VH, Louie A, Lomaestro BM, Drusano GL. 2003. Integration of population pharmacokinetics, a pharmacodynamic target, and microbiologic surveillance data to generate a rational empiric dosing strategy for cefepime against Pseudomonas aeruginosa. Pharmacotherapy 23:291-295. https://doi.org/10.1592/phco.23.3.291.32110.

9. Nicasio AM, VanScoy BD, Mendes RE, Castanheira M, Bulik CC, Okusanya OO, Bhavnani SM, Forrest A, Jones RN, Friedrich LV, Steenbergen JN Ambrose PG. 2016. Pharmacokinetics-pharmacodynamics of tazobactam in combination with piperacillin in an in vitro infection model. Antimicrob Agents Chemother 60:2075-2080. https://doi.org/10.1128/ AAC.02747-15.

10. Vanscoy B, Mendes RE, Castanheira M, McCauley J, Bhavnani SM, Forrest A, Jones RN, Okusanya OO, Friedrich LV, Steenbergen J, Am- brose PG. 2013. Relationship between ceftolozane-tazobactam exposure and drug resistance amplification in a hollow-fiber infection model. Antimicrob Agents Chemother 57:4134-4138. https://doi.org/ 10.1128/AAC.00461-13.

11. VanScoy BD, Tenero D, Turner S, Livermore DM, McCauley J, Conde $H$, Bhavnani SM, Rubino CM, Ambrose PG. 2017. Pharmacokineticspharmacodynamics of tazobactam in combination with cefepime in an in vitro infection model. Antimicrob Agents Chemother 61:e01052-17. https://doi.org/10.1128/AAC.01052-17.

12. Bhagunde $P$, Chang K-T, Hirsch EB, Ledesma KR, Nikolaou M, Tam VH. 2012. Novel modeling framework to guide design of optimal dosing strategies for $\beta$-lactamase inhibitors. Antimicrob Agents Chemother 56:2237-2240. https://doi.org/10.1128/AAC.06113-11.

13. Sy SKB, Zhuang L, Sy S, Derendorf H. 2019. Clinical pharmacokinetics and pharmacodynamics of ceftazidime-avibactam combination: a model-informed strategy for its clinical development. Clin Pharmacokinet 58:545-564. https://doi.org/10.1007/s40262-018-0705-y.

14. FDA. 2017. Meropenem-vaborbactam. Center for Drug Evaluation and Research. Application number 2097760rig1s000, clinical pharmacology and biopharmaceutics review(s). FDA, Silver Spring, MD.

15. FDA. 2018. Imipenem-relebactam. Center for Drug Evaluation and Research. Application number 2128190rig1s000, multi-discipline review. FDA, Silver Spring, MD.

16. Abodakpi $H$, Wanger $A$, Tam VH. 2019. What the clinical microbiologist should know about pharmacokinetics/pharmacodynamics in the era of emerging multidrug resistance: focusing on $\beta$-lactam/ $\beta$-lactamase inhibitor combinations. Clin Lab Med 39:473-485. https://doi.org/10 1016/j.cll.2019.05.006

17. Veiga RP, Paiva J-A. 2018. Pharmacokinetics-pharmacodynamics issues relevant for the clinical use of beta-lactam antibiotics in critically il patients. Crit Care 22:233. https://doi.org/10.1186/s13054-018-2155-1.

18. Phe K, Heil E, Tam V. 2020. Optimizing pharmacokinetics/pharmacodynamics of antimicrobial management in patients with sepsis. J Infect Dis 222:S132-S141. https://doi.org/10.1093/infdis/jiaa118.

19. Lagacé-Wiens P, Walkty A, Karlowsky JA. 2014. Ceftazidime-avibactam: an evidence-based review of its pharmacology and potential use in the treatment of Gram-negative bacterial infections. Core Evid 9:13-25. https://doi.org/10.2147/CE.S40698.

20. Li H, Estabrook M, Jacoby GA, Nichols WW, Testa RT, Bush K. 2015. In vitro susceptibility of characterized $\beta$-lactamase-producing strains tested with avibactam combinations. Antimicrob Agents Chemother 59:1789-1793. https://doi.org/10.1128/AAC.04191-14.

21. Carmeli Y, Armstrong J, Laud PJ, Newell P, Stone G, Wardman A, Gasink LB. 2016. Ceftazidime-avibactam or best available therapy in patients 
with ceftazidime-resistant Enterobacteriaceae and Pseudomonas aeruginosa complicated urinary tract infections or complicated intraabdominal infections (REPRISE): a randomised, pathogen-directed, phase 3 study. Lancet Infect Dis 16:661-673. https://doi.org/10.1016/ S1473-3099(16)30004-4.

22. Sader HS, Castanheira M, Mendes RE, Flamm RK. 2018. Frequency and antimicrobial susceptibility of Gram-negative bacteria isolated from patients with pneumonia hospitalized in ICUs of US medical centres (2015-17). J Antimicrob Chemother 73:3053-3059. https://doi.org/10 .1093/jac/dky279.

23. Vasoo S, Cunningham SA, Cole NC, Kohner PC, Menon SR, Krause KM, Harris KA, De PP, Koh TH, Patel R. 2015. In vitro activities of ceftazidimeavibactam, aztreonam-avibactam, and a panel of older and contemporary antimicrobial agents against carbapenemase-producing Gramnegative bacilli. Antimicrob Agents Chemother 59:7842-7846. https:// doi.org/10.1128/AAC.02019-15.

24. Sader HS, Castanheira M, Shortridge D, Mendes RE, Flamm RK. 2017. Antimicrobial activity of ceftazidime-avibactam tested against multidrug-resistant Enterobacteriaceae and Pseudomonas aeruginosa isolates from U.S. medical centers, 2013 to 2016. Antimicrob Agents Chemother 61:e01045-17. https://doi.org/10.1128/AAC.01045-17.

25. Citron DM, Tyrrell KL, Merriam V, Goldstein EJC. 2011. In vitro activity of ceftazidime-NXL104 against 396 strains of beta-lactamase-producing anaerobes. Antimicrob Agents Chemother 55:3616-3620. https://doi .org/10.1128/AAC.01682-10.

26. Rasmussen BA, Bush K, Tally FP. 1997. Antimicrobial resistance in anaerobes. Clin Infect Dis 24(Suppl 1):S110-S120. https://doi.org/10 .1093/clinids/24.Supplement_1.S110.

27. Castanheira M, Farrell SE, Krause KM, Jones RN, Sader HS. 2014. Contemporary diversity of $\beta$-lactamases among Enterobacteriaceae in the nine U.S. census regions and ceftazidime-avibactam activity tested against isolates producing the most prevalent $\beta$-lactamase groups. Antimicrob Agents Chemother 58:833-838. https://doi.org/10.1128/ AAC.01896-13.

28. López-Hernández I, Alonso N, Fernández-Martínez $M$, Zamorano $L$, Rivera A, Oliver A, Conejo MC, Martínez-Martínez L, Navarro F, Pascual A. 2017. Activity of ceftazidime-avibactam against multidrug-resistance Enterobacteriaceae expressing combined mechanisms of resistance. Enferm Infecc Microbiol Clin 35:499-504. https://doi.org/10.1016/j.eimc .2016.09.013.

29. Testa R, Cantón R, Giani T, Morosini M-I, Nichols WW, Seifert H, Stefanik D, Rossolini GM, Nordmann P. 2015. In vitro activity of ceftazidime, ceftaroline and aztreonam alone and in combination with avibactam against European Gram-negative and Gram-positive clinical isolates. Int J Antimicrob Agents 45:641-646. https://doi.org/10.1016/j.ijantimicag .2014.12.033.

30. Sader HS, Castanheira M, Flamm RK. 2017. Antimicrobial activity of ceftazidime-avibactam against Gram-negative bacteria isolated from patients hospitalized with pneumonia in U.S. medical centers, 2011 to 2015. Antimicrob Agents Chemother 61:e02083-16. https://doi.org/10 .1128/AAC.02083-16.

31. Livermore DM, Meunier D, Hopkins KL, Doumith M, Hill R, Pike R, Staves P, Woodford N. 2018. Activity of ceftazidime/avibactam against problem Enterobacteriaceae and Pseudomonas aeruginosa in the UK, 2015-16. J Antimicrob Chemother 73:648-657. https://doi.org/10 .1093/jac/dkx438.

32. Alraddadi BM, Saeedi M, Qutub M, Alshukairi A, Hassanien A, Wali G. 2019. Efficacy of ceftazidime-avibactam in the treatment of infections due to carbapenem-resistant Enterobacteriaceae. BMC Infect Dis 19:772. https://doi.org/10.1186/s12879-019-4409-1.

33. De la Calle C, Rodríguez O, Morata L, Marco F, Cardozo C, García-Vidal C, Río AD, Feher C, Pellicé M, Puerta-Alcalde P, Mensa J, Soriano A, Martínez JA. 2019. Clinical characteristics and prognosis of infections caused by OXA-48 carbapenemase-producing Enterobacteriaceae in patients treated with ceftazidime-avibactam. Int J Antimicrob Agents 53:520-524. https://doi.org/10.1016/j.ijantimicag.2018.11.015.

34. Haidar G, Clancy CJ, Shields RK, Hao B, Cheng S, Nguyen MH. 2017. Mutations in $b / a_{\mathrm{KPC}-3}$ that confer ceftazidime-avibactam resistance encode novel KPC-3 variants that function as extended-spectrum $\beta$-lactamases. Antimicrob Agents Chemother 61:e02534-16. https://doi .org/10.1128/AAC.02534-16.

35. Papp-Wallace KM, Becka SA, Zeiser ET, Ohuchi N, Mojica MF, Gatta JA, Falleni M, Tosi D, Borghi E, Winkler ML, Wilson BM, LiPuma JJ, Nukaga M, Bonomo RA. 2017. Overcoming an extremely drug resistant (XDR) pathogen: avibactam restores susceptibility to ceftazidime for Burkholderia cepacia complex isolates from cystic fibrosis patients. ACS Infect Dis 3:502-511. https://doi.org/10.1021/acsinfecdis.7b00020.

36. Karlowsky JA, Biedenbach DJ, Kazmierczak KM, Stone GG, Sahm DF. 2016. Activity of ceftazidime-avibactam against extended-spectrumand AmpC $\beta$-lactamase-producing Enterobacteriaceae collected in the INFORM global surveillance study from 2012 to 2014. Antimicrob Agents Chemother 60:2849-2857. https://doi.org/10.1128/AAC .02286-15.

37. Sader HS, Castanheira M, Duncan LR, Flamm RK. 2018. Antimicrobial susceptibility of Enterobacteriaceae and Pseudomonas aeruginosa isolates from United States medical centers stratified by infection type: results from the international network for optimal resistance monitoring (INFORM) surveillance program, 2015-2016. Diagn Microbiol Infect Dis 92:69-74. https://doi.org/10.1016/j.diagmicrobio.2018.04.012.

38. Spiliopoulou I, Kazmierczak K, Stone GG. 2020. In vitro activity of ceftazidime/avibactam against isolates of carbapenem-non-susceptible Enterobacteriaceae collected during the INFORM global surveillance programme (2015-17). J Antimicrob Chemother 75:384-391. https:// doi.org/10.1093/jac/dkz456.

39. Galani I, Karaiskos I, Karantani I, Papoutsaki V, Maraki S, Papaioannou V, Kazila P, Tsorlini H, Charalampaki N, Toutouza M, Vagiakou H, Pappas K, Kyratsa A, Kontopoulou K, Legga O, Petinaki E, Papadogeorgaki H, Chinou E, Souli M, Giamarellou H, Study Collaborators. 2018. Epidemiology and resistance phenotypes of carbapenemase-producing Klebsiella pneumoniae in Greece, 2014 to 2016. Euro Surveill 23:1700775. https://doi.org/10.2807/1560-7917.ES.2018.23.30.1700775.

40. Castanheira M, Mendes RE, Sader HS. 2017. Low frequency of ceftazidime-avibactam resistance among Enterobacteriaceae isolates carrying $b / a_{\mathrm{KPC}}$ collected in U.S. hospitals from 2012 to 2015. Antimicrob Agents Chemother 61:e02369-16. https://doi.org/10.1128/ AAC.02369-16.

41. Yasmin M, Fouts DE, Jacobs MR, Haydar $H$, Marshall SH, White R, D'Souza R, Lodise TP, Rhoads DD, Hujer AM, Rojas L, Hoyen C, Perez F, Edwards A, Bonomo RA. 2020. Monitoring ceftazidime-avibactam and aztreonam concentrations in the treatment of a bloodstream infection caused by a multidrug-resistant Enterobacter sp. carrying both Klebsiella pneumoniae carbapenemase- 4 and New Delhi metallo- $\beta$-lactamase- 1 . Clin Infect Dis 71:1095-1098. https://doi.org/10.1093/cid/ciz1155.

42. Mojica MF, Ouellette CP, Leber A, Becknell MB, Ardura MI, Perez F, Shimamura M, Bonomo RA, Aitken SL, Shelburne SA. 2016. Successful treatment of bloodstream infection due to metallo- $\beta$-lactamaseproducing Stenotrophomonas maltophilia in a renal transplant patient. Antimicrob Agents Chemother 60:5130-5134. https://doi.org/10.1128/ AAC.00264-16.

43. Flamm RK, Farrell DJ, Sader HS, Jones RN. 2014. Ceftazidime/avibactam activity tested against Gram-negative bacteria isolated from bloodstream, pneumonia, intra-abdominal and urinary tract infections in US medical centres (2012). J Antimicrob Chemother 69:1589-1598. https:// doi.org/10.1093/jac/dku025.

44. Chalhoub H, Tunney M, Elborn JS, Vergison A, Denis O, Plésiat P, Kahl BC, Van Bambeke F, Tulkens PM. 2015. Avibactam confers susceptibility to a large proportion of ceftazidime-resistant Pseudomonas aeruginosa isolates recovered from cystic fibrosis patients. J Antimicrob Chemother 70:1596-1598. https://doi.org/10.1093/jac/dku551.

45. Sader HS, Castanheira M, Mendes RE, Flamm RK, Farrell DJ, Jones RN 2015. Ceftazidime-avibactam activity against multidrug-resistant Pseudomonas aeruginosa isolated in U.S. medical centers in 2012 and 2013. Antimicrob Agents Chemother 59:3656-3659. https://doi.org/10.1128/ AAC.05024-14.

46. Buehrle DJ, Shields RK, Chen L, Hao B, Press EG, Alkrouk A, Potoski BA, Kreiswirth BN, Clancy CJ, Nguyen MH. 2016. Evaluation of the in vitro activity of ceftazidime-avibactam and ceftolozane-tazobactam against meropenem-resistant Pseudomonas aeruginosa isolates. Antimicrob Agents Chemother 60:3227-3231. https://doi.org/10.1128/ AAC.02969-15.

47. Alatoom A, Elsayed H, Lawlor K, AbdelWareth L, El-Lababidi R, Cardona L, Mooty M, Bonilla M-F, Nusair A, Mirza I. 2017. Comparison of antimicrobial activity between ceftolozane-tazobactam and ceftazidimeavibactam against multidrug-resistant isolates of Escherichia coli, Klebsiella pneumoniae, and Pseudomonas aeruginosa. Int J Infect Dis 62: 39-43. https://doi.org/10.1016/j.ijid.2017.06.007.

48. Zamudio R, Hijazi K, Joshi C, Aitken E, Oggioni MR, Gould IM. 2019. Phylogenetic analysis of resistance to ceftazidime/avibactam, ceftolozane/ 
tazobactam and carbapenems in piperacillin/tazobactam-resistant Pseudomonas aeruginosa from cystic fibrosis patients. Int J Antimicrob Agents 53:774-780. https://doi.org/10.1016/j.ijantimicag.2019.02.022.

49. Schaumburg F, Bletz S, Mellmann A, Becker K, Idelevich EA. 2019. Comparison of methods to analyse susceptibility of German MDR/XDR Pseudomonas aeruginosa to ceftazidime/avibactam. Int J Antimicrob Agents 54:255-260. https://doi.org/10.1016/j.ijantimicag.2019.05.001.

50. Ehmann DE, Jahic $H$, Ross PL, Gu R-F, Hu J, Durand-Réville TF, Lahiri $S$, Thresher J, Livchak S, Gao N, Palmer T, Walkup GK, Fisher SL. 2013. Kinetics of avibactam inhibition against class $A, C$, and $D \beta$-lactamases. J Biol Chem 288:27960-27971. https://doi.org/10.1074/jbc.M113.485979.

51. Nelson K, Hemarajata P, Sun D, Rubio-Aparicio D, Tsivkovski R, Yang S, Sebra R, Kasarskis A, Nguyen H, Hanson BM, Leopold S, Weinstock G, Lomovskaya O, Humphries RM. 2017. Resistance to ceftazidimeavibactam is due to transposition of KPC in a porin-deficient strain of Klebsiella pneumoniae with increased efflux activity. Antimicrob Agents Chemother 61:e00989-17. https://doi.org/10.1128/AAC.00989-17.

52. Humphries RM, Hemarajata P. 2017. Resistance to ceftazidimeavibactam in Klebsiella pneumoniae due to porin mutations and the increased expression of KPC-3. Antimicrob Agents Chemother 61: e00537-17. https://doi.org/10.1128/AAC.00537-17.

53. Zhang Y, Kashikar A, Brown CA, Denys G, Bush K. 2017. Unusual Escherichia coli PBP 3 insertion sequence identified from a collection of carbapenem-resistant Enterobacteriaceae tested in vitro with a combination of ceftazidime-, ceftaroline-, or aztreonam-avibactam. Antimicrob Agents Chemother 61:e00389-17. https://doi.org/10.1128/AAC .00389-17.

54. Both $A$, Büttner $H$, Huang J, Perbandt M, Belmar Campos C, Christner $M$, Maurer FP, Kluge S, König C, Aepfelbacher M, Wichmann D, Rohde H. 2017. Emergence of ceftazidime/avibactam non-susceptibility in an MDR Klebsiella pneumoniae isolate. J Antimicrob Chemother 72: 2483-2488. https://doi.org/10.1093/jac/dkx179.

55. Giddins MJ, Macesic N, Annavajhala MK, Stump S, Khan S, McConville TH, Mehta M, Gomez-Simmonds A, Uhlemann A-C. 2017. Successive emergence of ceftazidime-avibactam resistance through distinct genomic adaptations in $b / a_{\mathrm{KPC}-2}$-harboring Klebsiella pneumoniae sequence type 307 isolates. Antimicrob Agents Chemother 62:e02101-17. https://doi.org/10.1128/AAC.02101-17.

56. Gaibani P, Campoli C, Lewis RE, Volpe SL, Scaltriti E, Giannella M, Pongolini S, Berlingeri A, Cristini F, Bartoletti M, Tedeschi S, Ambretti S. 2018. In vivo evolution of resistant subpopulations of KPC-producing Klebsiella pneumoniae during ceftazidime/avibactam treatment. J Antimicrob Chemother 73:1525-1529. https://doi.org/10.1093/jac/dky082.

57. Sun L, Chen W, Li H, Li L, Zou X, Zhao J, Lu B, Li B, Wang C, Li H, Liu Y, Cao B. 2020. Phenotypic and genotypic analysis of KPC-51 and KPC-52, two novel KPC-2 variants conferring resistance to ceftazidime/ avibactam in the KPC-producing Klebsiella pneumoniae ST11 clone background. J Antimicrob Chemother Jul 75:3072-3074. https://doi .org/10.1093/jac/dkaa241.

58. Livermore DM, Jamrozy D, Mushtaq S, Nichols WW, Young K, Woodford N. 2017. AmpC $\beta$-lactamase induction by avibactam and relebactam. J Antimicrob Chemother 72:3342-3348. https://doi.org/10 .1093/jac/dkx298.

59. Miossec C, Claudon M, Levasseur P, Black MT. 2013. The $\beta$-lactamase inhibitor avibactam (NXL104) does not induce ampC $\beta$-lactamase in Enterobacter cloacae. Infect Drug Resist 6:235-240. https://doi.org/10 .2147/IDR.S53874.

60. Galani I, Karaiskos I, Souli M, Papoutsaki V, Galani L, Gkoufa A, Antoniadou A, Giamarellou H. 2020. Outbreak of KPC-2-producing Klebsiella pneumoniae endowed with ceftazidime-avibactam resistance mediated through a VEB-1-mutant (VEB-25), Greece, September to October 2019. Euro Surveill 25:2000028. https://doi.org/10.2807/1560-7917.ES.2020 25.3.2000028.

61. Winkler ML, Papp-Wallace KM, Hujer AM, Domitrovic TN, Hujer KM, Hurless KN, Tuohy M, Hall G, Bonomo RA. 2015. Unexpected challenges in treating multidrug-resistant Gram-negative bacteria: resistance to ceftazidime-avibactam in archived isolates of Pseudomonas aeruginosa. Antimicrob Agents Chemother 59:1020-1029. https://doi.org/10.1128/ AAC.04238-14.

62. Grupper M, Sutherland C, Nicolau DP. 2017. Multicenter evaluation of ceftazidime-avibactam and ceftolozane-tazobactam inhibitory activity against meropenem-nonsusceptible Pseudomonas aeruginosa from blood, respiratory tract, and wounds. Antimicrob Agents Chemother 61:e00875-17. https://doi.org/10.1128/AAC.00875-17.
63. Aitken SL, Tarrand JJ, Deshpande LM, Tverdek FP, Jones AL, Shelburne SA, Prince RA, Bhatti MM, Rolston KVI, Jones RN, Castanheira M, Chemaly RF. 2016. High rates of nonsusceptibility to ceftazidime-avibactam and identification of New Delhi metallo- $\beta$-lactamase production in Enterobacteriaceae bloodstream infections at a major cancer center. Clin Infect Dis 63:954-958. https://doi.org/10.1093/cid/ciw398.

64. Lahiri SD, Walkup GK, Whiteaker JD, Palmer T, McCormack K, Tanudra MA, Nash TJ, Thresher J, Johnstone MR, Hajec L, Livchak S, McLaughlin RE, Alm RA. 2015. Selection and molecular characterization of ceftazidime/avibactam-resistant mutants in Pseudomonas aeruginosa strains containing derepressed AmpC. J Antimicrob Chemother 70: 1650-1658. https://doi.org/10.1093/jac/dkv004.

65. Mushtaq S, Warner M, Livermore DM. 2010. In vitro activity of ceftazidime +NXL104 against Pseudomonas aeruginosa and other nonfermenters. J Antimicrob Chemother 65:2376-2381. https://doi.org/10 $.1093 / \mathrm{jac} / \mathrm{dkq} 306$.

66. Curcio D. 2011. Activity of a novel combination against multidrugresistant nonfermenters: ceftazidime plus NXL104. Expert Rev Anti Infect Ther 9:173-176. https://doi.org/10.1586/eri.10.173.

67. Castanheira M, Arends SJR, Davis AP, Woosley LN, Bhalodi AA, MacVane SH. 2018. Analyses of a ceftazidime-avibactam-resistant Citrobacter freundii isolate carrying $b / a_{\mathrm{KPC}-2}$ reveals a heterogenous population and reversible genotype. mSphere 3:e00408-18. https://doi.org/10.1128/ mSphere.00408-18.

68. Shields RK, Chen L, Cheng S, Chavda KD, Press EG, Snyder A, Pandey R, Doi Y, Kreiswirth BN, Nguyen MH, Clancy CJ. 2017. Emergence of ceftazidime-avibactam resistance due to plasmid-borne $b a_{\mathrm{KPC}-3}$ mutations during treatment of carbapenem-resistant Klebsiella pneumoniae infections. Antimicrob Agents Chemother 61:e02097-16. https://doi .org/10.1128/AAC.02097-16.

69. Humphries RM, Yang S, Hemarajata P, Ward KW, Hindler JA, Miller SA, Gregson A. 2015. First report of ceftazidime-avibactam resistance in a KPC-3-expressing Klebsiella pneumoniae isolate. Antimicrob Agents Chemother 59:6605-6607. https://doi.org/10.1128/AAC.01165-15.

70. Shen Z, Ding B, Ye M, Wang P, Bi Y, Wu S, Xu X, Guo Q, Wang M. 2017. High ceftazidime hydrolysis activity and porin OmpK35 deficiency contribute to the decreased susceptibility to ceftazidime/avibactam in KPC-producing Klebsiella pneumoniae. J Antimicrob Chemother 72: 1930-1936. https://doi.org/10.1093/jac/dkx066.

71. Zhang P, Shi Q, Hu H, Hong B, Wu X, Du X, Akova M, Yu Y. 2020. Emergence of ceftazidime/avibactam resistance in carbapenemresistant Klebsiella pneumoniae in China. Clin Microbiol Infect 26: 124.e1-124.e4. https://doi.org/10.1016/j.cmi.2019.08.020.

72. Compain F, Arthur M. 2017. Impaired inhibition by avibactam and resistance to the ceftazidime-avibactam combination due to the D179Y substitution in the KPC-2 $\beta$-lactamase. Antimicrob Agents Chemother 61:e00451-17. https://doi.org/10.1128/AAC.00451-17.

73. Gaibani P, Re MC, Campoli C, Viale PL, Ambretti S. 2020. Bloodstream infection caused by KPC-producing Klebsiella pneumoniae resistant to ceftazidime/avibactam: epidemiology and genomic characterization. Clin Microbiol Infect 26:516.e1-516.e4. https://doi.org/10.1016/j.cmi .2019.11.011

74. Shields RK, Potoski BA, Haidar G, Hao B, Doi Y, Chen L, Press EG, Kreiswirth BN, Clancy CJ, Nguyen MH. 2016. Clinical outcomes, drug toxicity, and emergence of ceftazidime-avibactam resistance among patients treated for carbapenem-resistant Enterobacteriaceae infections. Clin Infect Dis 63:1615-1618. https://doi.org/10.1093/cid/ciw636.

75. Fraile-Ribot PA, Mulet X, Cabot G, Del Barrio-Tofiño E, Juan C, Pérez JL, Oliver A. 2017. In vivo emergence of resistance to novel cephalosporin$\beta$-lactamase inhibitor combinations through the duplication of amino acid D149 from OXA-2 $\beta$-lactamase (OXA-539) in sequence type 235 Pseudomonas aeruginosa. Antimicrob Agents Chemother 61:e01117-17. https://doi.org/10.1128/AAC.01117-17.

76. Allergan, Inc. 2019. Avycaz (package insert). Allergan, Inc., Irvine, CA.

77. Shields RK, Nguyen MH, Chen L, Press EG, Kreiswirth BN, Clancy CJ. 2018. Pneumonia and renal replacement therapy are risk factors for ceftazidime-avibactam treatment failures and resistance among patients with carbapenem-resistant Enterobacteriaceae infections. Antimicrob Agents Chemother 62:e02497-17. https://doi.org/10.1128/ AAC.02497-17.

78. European Medicines Agency. 2016. Zavicefta, INN-ceftazidime/avibactam. European Medicines Agency assessment report. European Medicines Agency, Amsterdam, Netherlands.

79. Mazuski JE, Gasink LB, Armstrong J, Broadhurst H, Stone GG, Rank D, 
Llorens L, Newell P, Pachl J. 2016. Efficacy and safety of ceftazidimeavibactam plus metronidazole versus meropenem in the treatment of complicated intra-abdominal infection: results from a randomized, controlled, double-blind, phase 3 program. Clin Infect Dis 62:1380-1389. https://doi.org/10.1093/cid/ciw133.

80. Sternbach N, Leibovici Weissman Y, Avni T, Yahav D. 2018. Efficacy and safety of ceftazidime/avibactam: a systematic review and metaanalysis. J Antimicrob Chemother 73:2021-2029. https://doi.org/10 .1093/jac/dky124.

81. Zhang Y, Tao L-N, Qu X-Y, Niu J-Q, Ding Y-H, Zhang S-X. 2018. Efficacy and safety of ceftazidime-avibactam in the treatment of complicated intra-abdominal infections (CIAls) and complicated urinary tract infections (CUTIs): a meta-analysis of randomized controlled trials. Rev Assoc Med Bras 64:253-263. https://doi.org/10.1590/1806-9282.64.03.253.

82. Che H, Wang R, Wang J, Cai Y. 2019. Ceftazidime/avibactam versus carbapenems for the treatment of infections caused by Enterobacteriaceae: a meta-analysis of randomised controlled trials. Int J Antimicrob Agents 54:809-813. https://doi.org/10.1016/j ijantimicag.2019.09.007.

83. Zhong H, Zhao X-Y, Zhang Z-L, Gu Z-C, Zhang C, Gao Y, Cui M. 2018. Evaluation of the efficacy and safety of ceftazidime/avibactam in the treatment of Gram-negative bacterial infections: a systematic review and meta-analysis. Int J Antimicrob Agents 52:443-450. https://doi.org/ 10.1016/j.jjantimicag.2018.07.004.

84. Stone GG, Newell P, Gasink LB, Broadhurst H, Wardman A, Yates K, Chen Z, Song J, Chow JW. 2018. Clinical activity of ceftazidime/avibactam against MDR Enterobacteriaceae and Pseudomonas aeruginosa: pooled data from the ceftazidime/avibactam phase III clinical trial programme. J Antimicrob Chemother 73:2519-2523. https://doi.org/10.1093/jac/ dky204.

85. Qin X, Tran BG, Kim MJ, Wang L, Nguyen DA, Chen Q, Song J, Laud PJ, Stone GG, Chow JW. 2017. A randomised, double-blind, phase 3 study comparing the efficacy and safety of ceftazidime/avibactam plus metronidazole versus meropenem for complicated intra-abdominal infections in hospitalised adults in Asia. Int J Antimicrob Agents 49:579-588. https://doi.org/10.1016/j.jjantimicag.2017.01.010.

86. Wagenlehner FM, Sobel JD, Newell P, Armstrong J, Huang X, Stone GG, Yates K, Gasink LB. 2016. Ceftazidime-avibactam versus doripenem for the treatment of complicated urinary tract infections, including acute pyelonephritis: RECAPTURE, a phase 3 randomized trial program. Clin Infect Dis 63:754-762. https://doi.org/10.1093/cid/ciw378.

87. Torres A, Zhong N, Pachl J, Timsit J-F, Kollef M, Chen Z, Song J, Taylor D, Laud PJ, Stone GG, Chow JW. 2018. Ceftazidime-avibactam versus meropenem in nosocomial pneumonia, including ventilator-associated pneumonia (REPROVE): a randomised, double-blind, phase 3 noninferiority trial. Lancet Infect Dis 18:285-295. https://doi.org/10.1016/ S1473-3099(17)30747-8.

88. Jorgensen SCJ, Trinh TD, Zasowski EJ, Lagnf AM, Bhatia S, Melvin SM, Steed ME, Simon SP, Estrada SJ, Morrisette T, Claeys KC, Rosenberg JR, Davis SL, Rybak MJ. 2019. Real-world experience with ceftazidimeavibactam for multidrug-resistant Gram-negative bacterial infections. Open Forum Infect Dis 6:ofz522. https://doi.org/10.1093/ofid/ofz522.

89. Rodríguez-Núñez $O$, Ripa $M$, Morata $L$, de la Calle $C$, Cardozo $C$, Fehér C, Pellicé M, Valcárcel A, Puerta-Alcalde P, Marco F, García-Vidal C, Del Río A, Soriano A, Martínez-Martínez JA. 2018. Evaluation of ceftazidime/ avibactam for serious infections due to multidrug-resistant and extensively drug-resistant Pseudomonas aeruginosa. J Glob Antimicrob Resist 15:136-139. https://doi.org/10.1016/j.jgar.2018.07.010.

90. van Duin D, Lok JJ, Earley M, Cober E, Richter SS, Perez F, Salata RA, Kalayjian RC, Watkins RR, Doi Y, Kaye KS, Fowler VG, Paterson DL, Bonomo RA, Evans S, Antibacterial Resistance Leadership Group. 2018. Colistin versus ceftazidime-avibactam in the treatment of infections due to carbapenem-resistant Enterobacteriaceae. Clin Infect Dis 66: 163-171. https://doi.org/10.1093/cid/cix783.

91. Tsolaki V, Mantzarlis K, Mpakalis A, Malli E, Tsimpoukas F, Tsirogianni A, Papagiannitsis C, Zygoulis P, Papadonta M-E, Petinaki E, Makris D, Zakynthinos E. 2019. Ceftazidime-avibactam to treat life-threatening infections by carbapenem-resistant pathogens in critically ill mechanically ventilated patients. Antimicrob Agents Chemother 64:e02320-19. https://doi.org/10.1128/AAC.02320-19.

92. Shields RK, Nguyen MH, Chen L, Press EG, Potoski BA, Marini RV, Doi Y, Kreiswirth BN, Clancy CJ. 2017. Ceftazidime-avibactam is superior to other treatment regimens against carbapenem-resistant Klebsiella pneumoniae bacteremia. Antimicrob Agents Chemother 61:e00883-17. https://doi.org/10.1128/AAC.00883-17.

93. King M, Heil E, Kuriakose S, Bias T, Huang V, El-Beyrouty C, McCoy D, Hiles J, Richards L, Gardner J, Harrington N, Biason K, Gallagher JC. 2017. Multicenter study of outcomes with ceftazidime-avibactam in patients with carbapenem-resistant Enterobacteriaceae infections. Antimicrob Agents Chemother 61:e00449-17. https://doi.org/10.1128/AAC .00449-17.

94. Onorato L, Di Caprio G, Signoriello S, Coppola N. 2019. Efficacy of ceftazidime/avibactam in monotherapy or combination therapy against carbapenem-resistant Gram-negative bacteria: a metaanalysis. Int J Antimicrob Agents 54:735-740. https://doi.org/10 .1016/j.ijantimicag.2019.08.025.

95. Bradley JS, Broadhurst $H$, Cheng K, Mendez M, Newell P, Prchlik M, Stone GG, Talley AK, Tawadrous M, Wajsbrot D, Yates K, Zuzova A, Gardner A. 2019. Safety and efficacy of ceftazidime-avibactam plus metronidazole in the treatment of children $\geq 3$ months to $<18$ years with complicated intra-abdominal infection: results from a phase 2 , randomized, controlled trial. Pediatr Infect Dis J 38:816-824. https:// doi.org/10.1097/INF.0000000000002392.

96. Bradley JS, Roilides E, Broadhurst H, Cheng K, Huang L-M, MasCasullo V, Newell P, Stone GG, Tawadrous M, Wajsbrot D, Yates K, Gardner A. 2019. Safety and efficacy of ceftazidime-avibactam in the treatment of children $\geq 3$ months to $<18$ years with complicated urinary tract infection: results from a phase 2 randomized, controlled trial. Pediatr Infect Dis J 38:920-928. https://doi.org/10.1097/ INF.0000000000002395.

97. Iosifidis E, Chorafa E, Agakidou E, Kontou A, Violaki A, Volakli E, Christou E-I, Zarras C, Drossou-Agakidou V, Sdougka M, Roilides E. 2019. Use of ceftazidime-avibactam for the treatment of extensively drug-resistant or pan drug-resistant Klebsiella pneumoniae in neonates and children $<5$ years of age. Pediatr Infect Dis J 38:812-815. https://doi.org/10 .1097/INF.0000000000002344.

98. Guimarães T, Nouér SA, Martins RCR, Perdigão Neto LV, Martins WMBS, Narciso Barbosa AC, Ferreira ALP, Costa SF, Gales AC. 2019. Ceftazidimeavibactam as salvage therapy for infections caused by Enterobacteriales coresistant to carbapenems and polymyxins. Antimicrob Agents Chemother 63:e00528-19. https://doi.org/10.1128/AAC.00528-19.

99. Tamma PD, Fan Y, Bergman Y, Sick-Samuels AC, Hsu AJ, Timp W, Simner PJ, Prokesch BC, Greenberg DE. 2018. Successful treatment of persistent Burkholderia cepacia complex bacteremia with ceftazidimeavibactam. Antimicrob Agents Chemother 62:e02213-17. https://doi .org/10.1128/AAC.02213-17.

100. Daccò V, Claut L, Piconi S, Castellazzi L, Garbarino F, Teri A, Colombo C. 2019. Successful ceftazidime-avibactam treatment of post-surgery Burkholderia multivorans genomovar II bacteremia and brain abscesses in a young lung transplanted woman with cystic fibrosis. Transpl Infect Dis 21:e13082. https://doi.org/10.1111/tid.13082.

101. Los-Arcos I, Len O, Martín-Gómez MT, González-López JJ, Saéz-Giménez B, Deu M, Nuvials X, Ferrer R, Román A, Gavaldà J. 2019. Lung transplantation in two cystic fibrosis patients infected with previously pandrug-resistant Burkholderia cepacia complex treated with ceftazidime-avibactam. Infection 47:289-292. https://doi.org/10.1007/ s15010-018-1261-y.

102. Spoletini G, Etherington C, Shaw N, Clifton IJ, Denton M, Whitaker $P$, Peckham DG. 2019. Use of ceftazidime/avibactam for the treatment of MDR Pseudomonas aeruginosa and Burkholderia cepacia complex infections in cystic fibrosis: a case series. J Antimicrob Chemother 74: 1425-1429. https://doi.org/10.1093/jac/dky558.

103. Cantón-Bulnes ML, Hurtado Martínez Á, López-Cerero L, Arenzana Seisdedos Á, Merino-Bohorquez V, Garnacho-Montero J. 2019. A case of pan-resistant Burkholderia cepacia complex bacteremic pneumonia, after lung transplantation treated with a targeted combination therapy. Transpl Infect Dis 21:e13034. https://doi.org/10.1111/tid.13034.

104. Barlow G, Morice A. 2018. Successful treatment of resistant Burkholderia multivorans infection in a patient with cystic fibrosis using ceftazidime/ avibactam plus aztreonam. J Antimicrob Chemother 73:2270-2271. https://doi.org/10.1093/jac/dky136.

105. Caverly L, Spilker T, Kalikin LM, Stillwell T, Young C, Huang DB, LiPuma JJ. 2019. In vitro activities of $\beta$-lactam- $\beta$-lactamase inhibitor antimicrobial agents against cystic fibrosis respiratory pathogens. Antimicrob Agents Chemother 64:e01595-19. https://doi.org/10.1128/AAC.01595-19.

106. Deshpande D, Srivastava S, Chapagain ML, Lee PS, Cirrincione KN, Pasipanodya JG, Gumbo T. 2017. The discovery of ceftazidime/ 
avibactam as an anti-Mycobacterium avium agent. J Antimicrob Chemother 72:i36-i42. https://doi.org/10.1093/jac/dkx306.

107. Deshpande D, Srivastava S, Pasipanodya JG, Lee PS, Gumbo T. 2017. A novel ceftazidime/avibactam, rifabutin, tedizolid and moxifloxacin (CARTM) regimen for pulmonary Mycobacterium avium disease. J Antimicrob Chemother 72:i48-i53. https://doi.org/10.1093/jac/dkx307.

108. Deshpande D, Srivastava S, Chapagain M, Magombedze G, Martin KR, Cirrincione KN, Lee PS, Koeuth T, Dheda K, Gumbo T. 2017. Ceftazidime-avibactam has potent sterilizing activity against highly drug-resistant tuberculosis. Sci Adv 3:e1701102. https://doi.org/10 $.1126 /$ sciadv.1701102.

109. Iannaccone M, Boattini M, Bianco G, Corcione S, Cavallo R, Costa C. 2020. Ceftazidime-avibactam susceptible to resistant KPC-producing Enterobacterales bloodstream infections: an observational study. J Chemother 32:160-162. https://doi.org/10.1080/1120009X.2019.1709363.

110. ClinicalTrials.gov. 2019. Impact of ceftazidime/avibactam treatment vs better available therapy on mortality of patients with infections caused by carbapenem-resistant enterobacteria (CAVICOR). https://clinicaltrials .gov/ct2/show/NCT04167228.

111. Farrell DJ, Flamm RK, Sader HS, Jones RN. 2013. Antimicrobial activity of ceftolozane-tazobactam tested against Enterobacteriaceae and Pseudomonas aeruginosa with various resistance patterns isolated in U.S. hospitals (2011-2012). Antimicrob Agents Chemother 57:6305-6310. https://doi.org/10.1128/AAC.01802-13.

112. Takeda S, Ishii Y, Hatano K, Tateda K, Yamaguchi K. 2007. Stability of FR264205 against AmpC beta-lactamase of Pseudomonas aeruginosa. Int J Antimicrob Agents 30:443-445. https://doi.org/10.1016/j.ijantimicag.2007 .05.019.

113. Melchers MJB, van Mil A, Mouton JW. 2015. In vitro activity of ceftolozane alone and in combination with tazobactam against extended-spectrum- $\beta$-lactamase-harboring Enterobacteriaceae. Antimicrob Agents Chemother 59:4521-4525. https://doi.org/10.1128/ AAC.04498-14.

114. Sader HS, Farrell DJ, Castanheira M, Flamm RK, Jones RN. 2014. Antimicrobial activity of ceftolozane/tazobactam tested against Pseudomonas aeruginosa and Enterobacteriaceae with various resistance patterns isolated in European hospitals (2011-12). J Antimicrob Chemother 69:2713-2722. https://doi.org/10.1093/jac/dku184.

115. Tato M, García-Castillo M, Bofarull AM, Cantón R, CENIT Study Group. 2015. In vitro activity of ceftolozane/tazobactam against clinical isolates of Pseudomonas aeruginosa and Enterobacteriaceae recovered in Spanish medical centres: results of the CENIT study. Int J Antimicrob Agents 46:502-510. https://doi.org/10.1016/j.ijantimicag.2015.07.004.

116. Del Barrio-Tofiño $E$, Zamorano L, Cortes-Lara S, López-Causapé C, Sánchez-Diener I, Cabot G, Bou G, Martínez-Martínez L, Oliver A, GEMARA-SEIMC/REIPI Pseudomonas study Group. 2019. Spanish nationwide survey on Pseudomonas aeruginosa antimicrobial resistance mechanisms and epidemiology. J Antimicrob Chemother 74: 1825-1835. https://doi.org/10.1093/jac/dkz147.

117. Jean S-S, Lu M-C, Shi Z-Y, Tseng S-H, Wu T-S, Lu P-L, Shao P-L, Ko W-C, Wang F-D, Hsueh P-R. 2018. In vitro activity of ceftazidime-avibactam, ceftolozane-tazobactam, and other comparable agents against clinically important Gram-negative bacilli: results from the 2017 Surveillance of Multicenter Antimicrobial Resistance in Taiwan (SMART). Infect Drug Resist 11:1983-1992. https://doi.org/10.2147/IDR.S175679.

118. Walkty A, Karlowsky JA, Adam H, Baxter M, Lagacé-Wiens P, Hoban DJ, Zhanel GG. 2013. In vitro activity of ceftolozane-tazobactam against Pseudomonas aeruginosa isolates obtained from patients in Canadian hospitals in the CANWARD study, 2007 to 2012. Antimicrob Agents Chemother 57:5707-5709. https://doi.org/10.1128/AAC.01404-13.

119. Livermore DM, Mushtaq $S$, Meunier D, Hopkins $K L$, Hill R, Adkin R, Chaudhry A, Pike R, Staves P, Woodford N, BSAC Resistance Surveillance Standing Committee. 2017. Activity of ceftolozane/tazobactam against surveillance and 'problem' Enterobacteriaceae, Pseudomonas aeruginosa and non-fermenters from the British Isles. J Antimicrob Chemother 72:2278-2289. https://doi.org/10.1093/jac/dkx136.

120. García-Fernández S, García-Castillo M, Melo-Cristino J, Pinto MF, Gonçalves E, Alves V, Vieira AR, Ramalheira E, Sancho L, Diogo J, Ferreira R, Silva D, Chaves C, Pássaro L, Paixão L, Cantón R, STEP Study Group. 2020. In vitro activity of ceftolozane-tazobactam against Enterobacterales and Pseudomonas aeruginosa causing urinary, intra-abdominal and lower respiratory tract infections in intensive care units in Portugal: the STEP multicentre study. Int J Antimicrob Agents 55:105887. https:// doi.org/10.1016/j.ijantimicag.2020.105887.
121. Snydman DR, McDermott LA, Jacobus NV. 2014. Activity of ceftolozanetazobactam against a broad spectrum of recent clinical anaerobic isolates. Antimicrob Agents Chemother 58:1218-1223. https://doi.org/ 10.1128/AAC.02253-13.

122. Moya B, Zamorano L, Juan C, Ge Y, Oliver A. 2010. Affinity of the new cephalosporin CXA-101 to penicillin-binding proteins of Pseudomonas aeruginosa. Antimicrob Agents Chemother 54:3933-3937. https://doi .org/10.1128/AAC.00296-10.

123. Sutherland CA, Nicolau DP. 2015. Susceptibility profile of ceftolozane/ tazobactam and other parenteral antimicrobials against Escherichia coli, Klebsiella pneumoniae, and Pseudomonas aeruginosa from US hospitals. Clin Ther 37:1564-1571. https://doi.org/10.1016/j.clinthera.2015.05.501.

124. Carvalhaes CG, Castanheira M, Sader HS, Flamm RK, Shortridge D. 2019. Antimicrobial activity of ceftolozane-tazobactam tested against gramnegative contemporary (2015-2017) isolates from hospitalized patients with pneumonia in US medical centers. Diagn Microbiol Infect Dis 94:93-102. https://doi.org/10.1016/j.diagmicrobio.2018.11.021.

125. Pfaller MA, Shortridge D, Sader HS, Castanheira M, Flamm RK. 2018. Ceftolozane/tazobactam activity against drug-resistant Enterobacteriaceae and Pseudomonas aeruginosa causing healthcare-associated infections in the Asia-Pacific region (minus China, Australia and New Zealand): report from an Antimicrobial Surveillance Programme (2013-2015). Int J Antimicrob Agents 51:181-189. https://doi.org/10 .1016/j.ijantimicag.2017.09.016.

126. Walkty A, Adam H, Baxter M, Lagacé-Wiens P, Karlowsky JA, Hoban DJ, Zhanel GG. 2018. In vitro activity of ceftolozane/tazobactam versus antimicrobial non-susceptible Pseudomonas aeruginosa clinical isolates including MDR and XDR isolates obtained from across Canada as part of the CANWARD study, 2008-16. J Antimicrob Chemother 73:703-708. https://doi.org/10.1093/jac/dkx468.

127. Shortridge D, Duncan LR, Pfaller MA, Flamm RK. 2019. Activity of ceftolozane-tazobactam and comparators when tested against Gramnegative isolates collected from paediatric patients in the USA and Europe between 2012 and 2016 as part of a global surveillance programme. Int J Antimicrob Agents 53:637-643. https://doi.org/10.1016/ j.ijantimicag.2019.01.015.

128. Yin D, Wu S, Yang Y, Shi Q, Dong D, Zhu D, Hu F. 2019. Results from the China Antimicrobial Surveillance Network (CHINET) in 2017 of the in vitro activities of ceftazidime-avibactam and ceftolozane-tazobactam against clinical isolates of Enterobacteriaceae and Pseudomonas aeruginosa. Antimicrob Agents Chemother 63:e02431-18. https://doi.org/10 .1128/AAC.02431-18.

129. Pazzini C, Ahmad-Nejad P, Ghebremedhin B. 2019. Ceftolozane/ tazobactam susceptibility testing in extended-spectrum betalactamaseand carbapenemase-producing Gram-negative bacteria of various clonal lineages. Eur J Microbiol Immunol (Bp) 9:1-4. https://doi.org/10.1556/1886 .2019.00001.

130. Haidar G, Philips NJ, Shields RK, Snyder D, Cheng S, Potoski BA, Doi Y, Hao B, Press EG, Cooper VS, Clancy CJ, Nguyen MH. 2017. Ceftolozanetazobactam for the treatment of multidrug-resistant Pseudomonas aeruginosa infections: clinical effectiveness and evolution of resistance. Clin Infect Dis 65:110-120. https://doi.org/10.1093/cid/cix182.

131. Skoglund E, Abodakpi H, Rios R, Diaz L, De La Cadena E, Dinh AQ, Ardila J, Miller WR, Munita JM, Arias CA, Tam VH, Tran TT. 2018. In vivo resistance to ceftolozane/tazobactam in Pseudomonas aeruginosa arising by AmpC- and non-AmpC-mediated pathways. Case Rep Infect Dis 2018:9095203. https://doi.org/10.1155/2018/9095203.

132. Fraile-Ribot PA, Cabot G, Mulet X, Periañez L, Martín-Pena ML, Juan C, Pérez JL, Oliver A. 2018. Mechanisms leading to in vivo ceftolozane/ tazobactam resistance development during the treatment of infections caused by MDR Pseudomonas aeruginosa. J Antimicrob Chemother 73:658-663. https://doi.org/10.1093/jac/dkx424.

133. Barnes MD, Taracila MA, Rutter JD, Bethel CR, Galdadas I, Hujer AM, Caselli E, Prati F, Dekker JP, Papp-Wallace KM, Haider S, Bonomo RA. 2018. Deciphering the evolution of cephalosporin resistance to ceftolozanetazobactam in Pseudomonas aeruginosa. mBio 9:e02085-18.

134. So W, Shurko J, Galega R, Quilitz R, Greene JN, Lee GC. 2019. Mechanisms of high-level ceftolozane/tazobactam resistance in Pseudomonas aeruginosa from a severely neutropenic patient and treatment success from synergy with tobramycin. J Antimicrob Chemother 74:269-271. https://doi.org/10.1093/jac/dky393.

135. Cabot G, Bruchmann S, Mulet X, Zamorano L, Moyà B, Juan C, Haussler S, Oliver A. 2014. Pseudomonas aeruginosa ceftolozane-tazobactam resistance development requires multiple mutations leading to over- 
expression and structural modification of AmpC. Antimicrob Agents Chemother 58:3091-3099. https://doi.org/10.1128/AAC.02462-13.

136. Jorgensen SCJ, Trinh TD, Zasowski EJ, Lagnf AM, Simon SP, Bhatia S, Melvin SM, Steed ME, Finch NA, Morrisette T, Estrada SJ, Rosenberg JR, Davis SL, Rybak MJ. 2020. Real-world experience with ceftolozanetazobactam for multidrug-resistant Gram-negative bacterial infections. Antimicrob Agents Chemother 64:e02291-19. https://doi.org/10.1128/ AAC.02291-19.

137. Buonomo AR, Maraolo AE, Scotto R, Foggia M, Zappulo E, Congera $P$, Parente S, Gentile I. 2020. Efficacy and safety of ceftolozane/ tazobactam as therapeutic option for complicated skin and soft tissue infections by MDR/XDR Pseudomonas aeruginosa in patients with impaired renal function: a case series from a single-center experience. Infection 48:303-307. https://doi.org/10.1007/s15010-020-01390-y.

138. Merck \& Co., Inc. 2019. Zerbaxa (package insert). Merck \& Co., Inc., Kenilworth, NJ.

139. Larson KB, Patel YT, Willavize S, Bradley JS, Rhee EG, Caro L, Rizk ML. 2019. Ceftolozane-tazobactam population pharmacokinetics and dose selection for further clinical evaluation in pediatric patients with complicated urinary tract or complicated intra-abdominal infections. Antimicrob Agents Chemother 63:e02578-18. https://doi.org/10.1128/AAC .02578-18.

140. Kollef MH, Nováček M, Kivistik Ü, Réa-Neto Á, Shime N, Martin-Loeches I, Timsit J-F, Wunderink RG, Bruno CJ, Huntington JA, Lin G, Yu B, Butterton JR, Rhee EG. 2019. Ceftolozane-tazobactam versus meropenem for treatment of nosocomial pneumonia (ASPECT-NP): a randomised, controlled, double-blind, phase 3, non-inferiority trial. Lancet Infect Dis 19:1299-1311. https://doi.org/10.1016/S1473-3099(19)30403-7.

141. Lucasti C, Hershberger E, Miller B, Yankelev S, Steenbergen J, Friedland I, Solomkin J. 2014. Multicenter, double-blind, randomized, phase II trial to assess the safety and efficacy of ceftolozane-tazobactam plus metronidazole compared with meropenem in adult patients with complicated intra-abdominal infections. Antimicrob Agents Chemother 58: 5350-5357. https://doi.org/10.1128/AAC.00049-14.

142. Solomkin J, Hershberger E, Miller B, Popejoy M, Friedland I, Steenbergen J, Yoon M, Collins S, Yuan G, Barie PS, Eckmann C. 2015. Ceftolozane/tazobactam plus metronidazole for complicated intraabdominal infections in an era of multidrug resistance: results from a randomized, double-blind, phase 3 trial (ASPECT-cIAI). Clin Infect Dis 60:1462-1471. https://doi.org/10.1093/cid/civ097.

143. Wagenlehner FM, Umeh O, Steenbergen J, Yuan G, Darouiche RO. 2015 Ceftolozane-tazobactam compared with levofloxacin in the treatment of complicated urinary-tract infections, including pyelonephritis: a randomised, double-blind, phase 3 trial (ASPECT-cUTI). Lancet 385: 1949-1956. https://doi.org/10.1016/S0140-6736(14)62220-0.

144. Cheng I-L, Chen Y-H, Lai C-C, Tang H-J. 2020. The use of ceftolozanetazobactam in the treatment of complicated intra-abdominal infections and complicated urinary tract infections-a meta-analysis of randomized controlled trials. Int J Antimicrob Agents 55:105858. https://doi.org/10 .1016/j.ijantimicag.2019.11.015.

145. Pogue JM, Kaye KS, Veve MP, Patel TS, Gerlach AT, Davis SL, Puzniak LA File TM, Olson S, Dhar S, Bonomo RA, Perez F. 2020. Ceftolozane/ tazobactam vs polymyxin or aminoglycoside-based regimens for the treatment of drug-resistant Pseudomonas aeruginosa. Clin Infect Dis 71:304-310. https://doi.org/10.1093/cid/ciz816.

146. Vena A, Giacobbe DR, Mussini C, Cattelan A, Bassetti M, for the Ceftabuse Study Group. 6 January 2020. Clinical efficacy of ceftolozanetazobactam versus other active agents for the treatment of bacteremia and nosocomial pneumonia due to drug-resistant Pseudomonas aeruginosa. Clin Infect Dis https://doi.org/10.1093/cid/ciaa003.

147. Gallagher JC, Satlin MJ, Elabor A, Saraiya N, McCreary EK, Molnar E, El-Beyrouty C, Jones BM, Dixit D, Heil EL, Claeys KC, Hiles J, Vyas NM, Bland CM, Suh J, Biason K, McCoy D, King MA, Richards L, Harrington N, Guo Y, Chaudhry S, Lu X, Yu D. 2018. Ceftolozane-tazobactam for the treatment of multidrug-resistant Pseudomonas aeruginosa infections: a multicenter study. Open Forum Infect Dis 5:ofy280. https://doi.org/10 .1093/ofid/ofy280.

148. Huntington JA, Sakoulas G, Umeh O, Cloutier DJ, Steenbergen JN, Bliss C, Goldstein EJC. 2016. Efficacy of ceftolozane/tazobactam versus levofloxacin in the treatment of complicated urinary tract infections (cUTIs) caused by levofloxacin-resistant pathogens: results from the ASPECTcUTI trial. J Antimicrob Chemother 71:2014-2021. https://doi.org/10 .1093/jac/dkw053.

149. Arakawa S, Kawahara K, Kawahara M, Yasuda M, Fujimoto G, Sato A,
Yokokawa R, Yoshinari T, Rhee EG, Aoyama N. 2019. The efficacy and safety of tazobactam/ceftolozane in Japanese patients with uncomplicated pyelonephritis and complicated urinary tract infection. J Infect Chemother 25:104-110. https://doi.org/10.1016/j.jiac.2018.10.009.

150. Mikamo H, Monden K, Miyasaka Y, Horiuchi T, Fujimoto G, Fukuhara T, Yoshinari T, Rhee EG, Shizuya T. 2019. The efficacy and safety of tazobactam/ceftolozane in combination with metronidazole in Japanese patients with complicated intra-abdominal infections. J Infect Chemother 25:111-116. https://doi.org/10.1016/j.jiac.2018.10.012.

151. Bradley JS, Ang JY, Arrieta AC, Larson KB, Rizk ML, Caro L, Yang S, Yu B, Johnson MG, Rhee EG. 2018. Pharmacokinetics and safety of single intravenous doses of ceftolozane/tazobactam in children with proven or suspected Gram-negative infection. Pediatr Infect Dis J 37: 1130-1136. https://doi.org/10.1097/INF.0000000000002170.

152. ClinicalTrials.gov. 2017. MK-7625A versus meropenem in pediatric participants with complicated urinary tract infection (cUTI) (MK-7625A034). https://clinicaltrials.gov/ct2/show/NCT03230838.

153. ClinicalTrials.gov. 2017. MK-7625A plus metronidazole versus meropenem in pediatric participants with complicated intra-abdominal infection (clAl) (MK-7625A-035). https://clinicaltrials.gov/ct2/show/ NCT03217136.

154. Zikri A, El Masri K. 2019. Use of ceftolozane/tazobactam for the treatment of multidrug-resistant Pseudomonas aeruginosa pneumonia in a pediatric patient with combined immunodeficiency (CID): a case report from a tertiary hospital in Saudi Arabia. Antibiotics (Basel) 8:67. https:// doi.org/10.3390/antibiotics8020067.

155. Aitken SL, Kontoyiannis DP, DePombo AM, Bhatti MM, Tverdek FP, Gettys SC, Nicolau DP, Nunez CA. 2016. Use of ceftolozane/tazobactam in the treatment of multidrug-resistant Pseudomonas aeruginosa bloodstream infection in a pediatric leukemia patient. Pediatr Infect Dis J 35:1040-1042. https://doi.org/10.1097/INF.0000000000001228.

156. Martín-Cazaña M, Grau S, Epalza C, Brañas P, Flores M, Olmedilla M, Blázquez-Gamero D. 2019. Successful ceftolozane-tazobactam rescue therapy in a child with endocarditis caused by multidrug-resistant Pseudomonas aeruginosa. J Paediatr Child Health 55:985-987. https:// doi.org/10.1111/jpc.14388.

157. Kullar R, Wagenlehner FM, Popejoy MW, Long J, Yu B, Goldstein EJC. 2017. Does moderate renal impairment affect clinical outcomes in complicated intra-abdominal and complicated urinary tract infections? Analysis of two randomized controlled trials with ceftolozane/ tazobactam. J Antimicrob Chemother 72:900-905. https://doi.org/10 $.1093 / \mathrm{jac} / \mathrm{dkw} 486$.

158. Bassetti M, Castaldo N, Cattelan A, Mussini C, Righi E, Tascini C, Menichetti F, Mastroianni CM, Tumbarello M, Grossi P, Artioli S, Carannante N, Cipriani L, Coletto D, Russo A, Digaetano M, Losito AR, Peghin M, Capone A, Nicolè S, Vena A, CEFTABUSE Study Group. 2019. Ceftolozane/tazobactam for the treatment of serious Pseudomonas aeruginosa infections: a multicentre nationwide clinical experience. Int J Antimicrob Agents 53:408-415. https://doi.org/10.1016/j.ijantimicag .2018.11.001.

159. Aguilar G, Ferriols R, Martínez-Castro S, Ezquer C, Pastor E, Carbonell JA, Alós M, Navarro D. 2019. Optimizing ceftolozane-tazobactam dosage in critically ill patients during continuous venovenous hemodiafiltration. Crit Care 23:145. https://doi.org/10.1186/s13054-019-2434-5.

160. Davis SE, Ham J, Hucks J, Gould A, Foster R, Justo JA, Nicolau DP, Bookstaver PB. 2019. Use of continuous infusion ceftolozanetazobactam with therapeutic drug monitoring in a patient with cystic fibrosis. Am J Health Syst Pharm 76:501-504. https://doi.org/10.1093/ ajhp/zxz011.

161. Popejoy MW, Long J, Huntington JA. 2017. Analysis of patients with diabetes and complicated intra-abdominal infection or complicated urinary tract infection in phase 3 trials of ceftolozane/tazobactam. BMC Infect Dis 17:316. https://doi.org/10.1186/s12879-017-2414-9.

162. Massip C, Mathieu C, Gaudru C, Miaut V, Floch P, Oswald E, Segonds C, Guet-Revillet H. 2019. In vitro activity of seven $\beta$-lactams including ceftolozane/tazobactam and ceftazidime/avibactam against Burkholderia cepacia complex, Burkholderia gladioli and other non-fermentative Gram-negative bacilli isolated from cystic fibrosis patients. J Antimicrob Chemother 74:525-528. https://doi.org/10.1093/jac/dky423.

163. Xipell M, Paredes S, Fresco L, Bodro M, Marco F, Martínez JA, Soriano A. 2018. Clinical experience with ceftolozane/tazobactam in patients with serious infections due to resistant Pseudomonas aeruginosa. J Glob Antimicrob Resist 13:165-170. https://doi.org/10.1016/j.jgar.2018.01.010.

164. Castón JJ, De la Torre Á, Ruiz-Camps I, Sorlí ML, Torres V, Torre-Cisneros 
J. 2017. Salvage therapy with ceftolozane-tazobactam for multidrugresistant Pseudomonas aeruginosa infections. Antimicrob Agents Chemother 61:e02136-16. https://doi.org/10.1128/AAC.02136-16.

165. Díaz-Cañestro $M$, Periañez $L$, Mulet $X$, Martin-Pena ML, Fraile-Ribot $P A$, Ayestarán I, Colomar A, Nuñez B, Maciá M, Novo A, Torres V, Asensio J, López-Causapé C, Delgado O, Pérez JL, Murillas J, Riera M, Oliver A. 2018. Ceftolozane/tazobactam for the treatment of multidrug resistant Pseudomonas aeruginosa: experience from the Balearic Islands. Eur J Clin Microbiol Infect Dis 37:2191-2200. https://doi.org/10.1007/s10096 -018-3361-0.

166. Escolà-Vergé L, Pigrau C, Los-Arcos I, Arévalo Á, Viñado B, Campany D, Larrosa N, Nuvials X, Ferrer R, Len O, Almirante B. 2018. Ceftolozane/ tazobactam for the treatment of XDR Pseudomonas aeruginosa infections. Infection 46:461-468. https://doi.org/10.1007/s15010-018-1133-5.

167. ClinicalTrials.gov. 2018. Comparative study to determine the efficacy, safety, and tolerability of ceftolozane-tazobactam. https://clinicaltrials .gov/ct2/show/NCT03485950.

168. ClinicalTrials.gov. 2019. Ceftolozane/tazobactam (MK-7625A) plus metronidazole versus meropenem for participants with complicated intra-abdominal infection (MK-7625A-015) (7625ACNPhase3). https:// clinicaltrials.gov/ct2/show/NCT03830333.

169. ClinicalTrials.gov. 2020. Ceftolozane-tazobactam versus meropenem for ESBL and AmpC-producing Enterobacterales bloodstream infection (MERINO III). https://clinicaltrials.gov/ct2/show/NCT04238390.

170. Hecker SJ, Reddy KR, Totrov M, Hirst GC, Lomovskaya O, Griffith DC, King P, Tsivkovski R, Sun D, Sabet M, Tarazi Z, Clifton MC, Atkins K, Raymond A, Potts KT, Abendroth J, Boyer SH, Loutit JS, Morgan EE, Durso S, Dudley MN. 2015. Discovery of a cyclic boronic acid $\beta$-lactamase inhibitor (RPX7009) with utility vs class A serine carbapenemases. J Med Chem 58:3682-3692. https://doi.org/10.1021/acs.jmedchem.5b00127.

171. Castanheira M, Rhomberg PR, Flamm RK, Jones RN. 2016. Effect of the $\beta$-lactamase inhibitor vaborbactam combined with meropenem against serine carbapenemase-producing Enterobacteriaceae. Antimicrob Agents Chemother 60:5454-5458. https://doi.org/10.1128/AAC .00711-16.

172. Castanheira M, Huband MD, Mendes RE, Flamm RK. 2017. Meropenemvaborbactam tested against contemporary Gram-negative isolates collected worldwide during 2014, including carbapenem-resistant, KPCproducing, multidrug-resistant, and extensively drug-resistant Enterobacteriaceae. Antimicrob Agents Chemother 61:e00567-17. https:// doi.org/10.1128/AAC.00567-17.

173. Livermore DM, Mushtaq S. 2013. Activity of biapenem (RPX2003) combined with the boronate $\beta$-lactamase inhibitor RPX7009 against carbapenem-resistant Enterobacteriaceae. J Antimicrob Chemother 68: 1825-1831. https://doi.org/10.1093/jac/dkt118.

174. Lapuebla A, Abdallah M, Olafisoye O, Cortes C, Urban C, Quale J, Landman D. 2015. Activity of meropenem combined with RPX7009, a novel $\beta$-lactamase inhibitor, against Gram-negative clinical isolates in New York City. Antimicrob Agents Chemother 59:4856-4860. https:// doi.org/10.1128/AAC.00843-15.

175. Bush K. 2015. A resurgence of $\beta$-lactamase inhibitor combinations effective against multidrug-resistant Gram-negative pathogens. Int J Antimicrob Agents 46:483-493. https://doi.org/10.1016/j.jjantimicag .2015.08.011.

176. Lomovskaya O, Sun D, Rubio-Aparicio D, Nelson K, Tsivkovski R, Griffith DC, Dudley MN. 2017. Vaborbactam: spectrum of beta-lactamase inhibition and impact of resistance mechanisms on activity in Enterobacteriaceae. Antimicrob Agents Chemother 61:e01443-17. https://doi.org/ 10.1128/AAC.01443-17.

177. Weiss WJ, Pulse ME, Nguyen P, Peterson K, Silva J, Simecka JW, Valtierra D, Sabet M, Griffith DC. 2017. Activity of meropenem-vaborbactam against carbapenem-resistant Enterobacteriaceae in a murine model of pyelonephritis. Antimicrob Agents Chemother 62:e01439-17. https:// doi.org/10.1128/AAC.01439-17.

178. Pfaller MA, Huband MD, Mendes RE, Flamm RK, Castanheira M. 2018. In vitro activity of meropenem/vaborbactam and characterisation of carbapenem resistance mechanisms among carbapenem-resistant Enterobacteriaceae from the 2015 meropenem/vaborbactam surveillance programme. Int J Antimicrob Agents 52:144-150. https://doi.org/10 .1016/j.ijantimicag.2018.02.021.

179. Thaden JT, Pogue JM, Kaye KS. 2017. Role of newer and re-emerging older agents in the treatment of infections caused by carbapenemresistant Enterobacteriaceae. Virulence 8:403-416. https://doi.org/10 $.1080 / 21505594.2016 .1207834$.
180. Hackel MA, Lomovskaya O, Dudley MN, Karlowsky JA, Sahm DF. 2017. In vitro activity of meropenem-vaborbactam against clinical isolates of KPC-positive Enterobacteriaceae. Antimicrob Agents Chemother 62: e01904-17. https://doi.org/10.1128/AAC.01904-17.

181. Kinn PM, Chen DJ, Gihring TM, Schulz LT, Fox BC, McCreary EK, Lepak AJ. 2019. In vitro evaluation of meropenem-vaborbactam against clinical CRE isolates at a tertiary care center with low KPC-mediated carbapenem resistance. Diagn Microbiol Infect Dis 93:258-260. https:// doi.org/10.1016/j.diagmicrobio.2018.09.017.

182. Carvalhaes CG, Shortridge D, Sader HS, Castanheira M. 2020. Activity of meropenem-vaborbactam against bacterial isolates causing pneumonia in hospitalized patients from U.S. hospitals during 2014 to 2018 Antimicrob Agents Chemother 64:e02177-19. https://doi.org/10.1128/ AAC.02177-19.

183. Sabet M, Tarazi Z, Rubio-Aparicio D, Nolan TG, Parkinson J, Lomovskaya O, Dudley MN, Griffith DC. 2017. Activity of simulated human dosage regimens of meropenem and vaborbactam against carbapenemresistant Enterobacteriaceae in an in vitro hollow-fiber model. Antimicrob Agents Chemother 62:e01969-17. https://doi.org/10.1128/AAC .01969-17.

184. Athans V, Neuner EA, Hassouna H, Richter SS, Keller G, Castanheira M, Brizendine KD, Mathers AJ. 2018. Meropenem-vaborbactam as salvage therapy for ceftazidime-avibactam-resistant Klebsiella pneumoniae bac teremia and abscess in a liver transplant recipient. Antimicrob Agents Chemother 63:e01551-18. https://doi.org/10.1128/AAC.01551-18.

185. Zhanel GG, Lawrence CK, Adam H, Schweizer F, Zelenitsky S, Zhanel M, Lagacé-Wiens PRS, Walkty A, Denisuik A, Golden A, Gin AS, Hoban DJ, Lynch JP, Karlowsky JA. 2018. Imipenem-relebactam and meropenemvaborbactam: two novel carbapenem- $\beta$-lactamase inhibitor combinations. Drugs 78:65-98. https://doi.org/10.1007/s40265-017-0851-9.

186. Castanheira M, Mendes RE, Duncan LR, Woosley LN, Flamm RK. 2017. Activity of meropenem-vaborbactam against Enterobacteriaceae isolates carrying $b / a_{\mathrm{KPC}}$ collected worldwide. Open Forum Infect Dis 4 (Suppl 1):S377. https://doi.org/10.1093/ofid/ofx163.930.

187. Melinta Therapeutics, Inc. 2018. VABOMERE (package insert). Melinta Therapeutics, Inc., Morristown, NJ.

188. Wenzler E, Gotfried MH, Loutit JS, Durso S, Griffith DC, Dudley MN, Rodvold KA. 2015. Meropenem-RPX7009 concentrations in plasma, epithelial lining fluid, and alveolar macrophages of healthy adult subjects. Antimicrob Agents Chemother 59:7232-7239. https://doi.org/10 .1128/AAC.01713-15.

189. Kaye KS, Bhowmick T, Metallidis S, Bleasdale SC, Sagan OS, Stus V, Vazquez J, Zaitsev V, Bidair M, Chorvat E, Dragoescu PO, Fedosiuk E, Horcajada JP, Murta C, Sarychev Y, Stoev V, Morgan E, Fusaro K, Griffith D, Lomovskaya O, Alexander EL, Loutit J, Dudley MN, GiamarellosBourboulis EJ. 2018. Effect of meropenem-vaborbactam vs piperacillintazobactam on clinical cure or improvement and microbial eradication in complicated urinary tract infection: the TANGO I Randomized Clinical Trial. JAMA 319:788-799. https://doi.org/10.1001/jama.2018.0438.

190. ClinicalTrials.gov. 2016. Dose-finding, pharmacokinetics, and safety of VABOMERE in pediatric subjects with bacterial infections (TANGOKIDS). https://clinicaltrials.gov/ct2/show/NCT02687906.

191. Hanretty AM, Kaur I, Evangelista AT, Moore WS, Enache A, Chopra A, Cies JJ. 2018. Pharmacokinetics of the meropenem component of meropenem-vaborbactam in the treatment of KPC-producing Klebsiella pneumoniae bloodstream infection in a pediatric patient. Pharmacotherapy 38:e87-e91. https://doi.org/10.1002/phar.2187.

192. Viale P. 2018. Meropenem-vaborbactam versus best-available therapy for carbapenem-resistant Enterobacteriaceae infections in TANGO II: outcomes in patients with cancer, abstract P0285. 28th European Society of Clinical Microbiology and Infectious Diseases, Madrid, Spain.

193. Wunderink RG, Giamarellos-Bourboulis EJ, Rahav G, Mathers AJ, Bassetti M, Vazquez J, Cornely OA, Solomkin J, Bhowmick T, Bishara J, Daikos GL, Felton T, Furst MJL, Kwak EJ, Menichetti F, Oren I, Alexander EL, Griffith D, Lomovskaya O, Loutit J, Zhang S, Dudley MN, Kaye KS. 2018. Effect and safety of meropenem-vaborbactam versus best-available therapy in patients with carbapenem-resistant Enterobacteriaceae infections: the TANGO II randomized clinical trial. Infect Dis Ther 7:439-455. https://doi.org/10.1007/s40121-018-0214-1.

194. Bassetti M, Giacobbe DR, Patel N, Tillotson G, Massey J. 2019. Efficacy and safety of meropenem-vaborbactam versus best available therapy for the treatment of carbapenem-resistant Enterobacteriaceae infections in patients without prior antimicrobial failure: a post hoc analysis. Adv Ther 36:1771-1777. https://doi.org/10.1007/s12325-019-00981-y. 
195. Lai C-C, Chen C-C, Tang H-J. 2019. Meropenem-vaborbactam in the treatment of acute bacterial infections. J Clin Med 8:1650. https://doi .org/10.3390/jcm8101650.

196. Shields RK, McCreary EK, Marini RV, Kline EG, Jones CE, Hao B, Chen L, Kreiswirth BN, Doi Y, Clancy CJ, Nguyen MH. 2020. Early experience with meropenem-vaborbactam for treatment of carbapenem-resistant Enterobacteriaceae infections. Clin Infect Dis 71:667-671. https://doi .org/10.1093/cid/ciz1131.

197. Jorgensen SCJ, McDonald P, Mynatt RP, Pogue JM, Lerner SA, Dhar S, Salimnia H, Rybak MJ. 2018. Averting the post-antibiotic era: successful use of meropenem/vaborbactam for carbapenemresistant Serratia marcescens and Enterobacter aerogenes bacteraemia in a haemodialysis patient. J Antimicrob Chemother 73: 3529-3531. https://doi.org/10.1093/jac/dky346.

198. Kufel WD, Eranki AP, Paolino KM, Call A, Miller CD, Mogle BT. 2019. In vivo pharmacokinetic analysis of meropenem/vaborbactam during continuous venovenous haemodialysis. J Antimicrob Chemother 74: 2117-2118. https://doi.org/10.1093/jac/dkz103.

199. Biagi M, Shajee A, Vialichka A, Jurkovic M, Tan X, Wenzler E. 2020. Activity of imipenem-relebactam and meropenem-vaborbactam against carbapenem-resistant SME-producing Serratia marcescens. Antimicrob Agents Chemother 64:e02255-19. https://doi.org/10.1128/ AAC.02255-19.

200. Sun D, Rubio-Aparicio D, Nelson K, Dudley MN, Lomovskaya O. 2017. Meropenem-vaborbactam resistance selection, resistance prevention, and molecular mechanisms in mutants of KPC-producing Klebsiella pneumoniae. Antimicrob Agents Chemother 61:e01694-17. https://doi .org/10.1128/AAC.01694-17.

201. ClinicalTrials.gov. 2016. A study of meropenem-vaborbactam versus piperacillin/tazobactam in participants with hospital-acquired and ventilator-associated bacterial pneumonia (TANGOIII). https://clinicaltrials .gov/ct2/show/NCT03006679.

202. Livermore DM, Warner M, Mushtaq S. 2013. Activity of MK-7655 combined with imipenem against Enterobacteriaceae and Pseudomonas aeruginosa. J Antimicrob Chemother 68:2286-2290. https://doi.org/10 $.1093 / \mathrm{jac} / \mathrm{dkt} 178$.

203. Olsen I. 2015. New promising $\beta$-lactamase inhibitors for clinical use. Eur J Clin Microbiol Infect Dis 34:1303-1308. https://doi.org/10.1007/ s10096-015-2375-0.

204. Young K, Painter RE, Raghoobar SL, Hairston NN, Racine F, Wisniewski D, Balibar CJ, Villafania A, Zhang R, Sahm DF, Blizzard T, Murgolo N, Hammond ML, Motyl MR. 2019. In vitro studies evaluating the activity of imipenem in combination with relebactam against Pseudomonas aeruginosa. BMC Microbiol 19:150. https://doi.org/10.1186/s12866-019 -1522-7.

205. Li H, Luo Y-F, Williams BJ, Blackwell TS, Xie C-M. 2012. Structure and function of OprD protein in Pseudomonas aeruginosa: from antibiotic resistance to novel therapies. Int J Med Microbiol 302:63-68. https:// doi.org/10.1016/j.ijmm.2011.10.001.

206. Schmidt-Malan SM, Mishra AJ, Mushtaq A, Brinkman CL, Patel R. 2018. In vitro activity of imipenem-relebactam and ceftolozane-tazobactam against resistant Gram-negative bacilli. Antimicrob Agents Chemother 62:e00533-18. https://doi.org/10.1128/AAC.00533-18.

207. Hirsch EB, Ledesma KR, Chang K-T, Schwartz MS, Motyl MR, Tam VH. 2012. In vitro activity of MK-7655, a novel $\beta$-lactamase inhibitor, in combination with imipenem against carbapenem-resistant Gramnegative bacteria. Antimicrob Agents Chemother 56:3753-3757. https://doi.org/10.1128/AAC.05927-11.

208. Karlowsky JA, Lob SH, Kazmierczak KM, Young K, Motyl MR, Sahm DF. 2018. In vitro activity of imipenem-relebactam against clinical isolates of Gram-negative bacilli isolated in hospital laboratories in the United States as part of the SMART 2016 program. Antimicrob Agents Chemother 62:e00169-18. https://doi.org/10.1128/AAC.00169-18.

209. Haidar G, Clancy CJ, Chen L, Samanta P, Shields RK, Kreiswirth BN, Nguyen MH. 2017. Identifying spectra of activity and therapeutic niches for ceftazidime-avibactam and imipenem-relebactam against carbapenem-resistant Enterobacteriaceae. Antimicrob Agents Chemother 61:e00642-17. https://doi.org/10.1128/AAC.00642-17.

210. Shields RK, Clancy CJ, Hao B, Chen L, Press EG, lovine NM, Kreiswirth BN, Nguyen MH. 2015. Effects of Klebsiella pneumoniae carbapenemase subtypes, extended-spectrum $\beta$-lactamases, and porin mutations on the in vitro activity of ceftazidime-avibactam against carbapenemresistant K. pneumoniae. Antimicrob Agents Chemother 59:5793-5797. https://doi.org/10.1128/AAC.00548-15.
211. Tooke $C L$, Hinchliffe $P$, Lang PA, Mulholland AJ, Brem J, Schofield $C J$, Spencer J. 2019. Molecular basis of class A $\beta$-lactamase inhibition by relebactam. Antimicrob Agents Chemother 63:e00564-19. https://doi .org/10.1128/AAC.00564-19.

212. Galani I, Souli M, Nafplioti K, Adamou P, Karaiskos I, Giamarellou H, Antoniadou A, study collaborators. 2019. In vitro activity of imipenem-relebactam against non-MBL carbapenemase-producing Klebsiella pneumoniae isolated in Greek hospitals in 2015-2016. Eur J Clin Microbiol Infect Dis 38:1143-1150. https://doi.org/10.1007/ s10096-019-03517-y.

213. Lob SH, Hackel MA, Kazmierczak KM, Young K, Motyl MR, Karlowsky JA, Sahm DF. 2017. In vitro activity of imipenem-relebactam against Gramnegative ESKAPE pathogens isolated by clinical laboratories in the United States in 2015 (results from the SMART global surveillance program). Antimicrob Agents Chemother 61:e02209-16. https://doi .org/10.1128/AAC.02209-16.

214. Karlowsky JA, Lob SH, Kazmierczak KM, Young K, Motyl MR, Sahm DF. 2020. In-vitro activity of imipenem/relebactam and key $\beta$-lactam agents against Gram-negative bacilli isolated from lower respiratory tract infection samples of intensive care unit patients - SMART surveillance United States 2015-2017. Int J Antimicrob Agents 55:105841. https:// doi.org/10.1016/j.ijantimicag.2019.10.022.

215. Karlowsky JA, Lob SH, Kazmierczak KM, Young K, Motyl MR, Sahm DF. 2020. In vitro activity of imipenem-relebactam against Enterobacteriaceae and Pseudomonas aeruginosa isolated from intraabdominal and urinary tract infection samples - SMART surveillance United States 2015-2017. J Glob Antimicrob Resist 21:223-217. https://doi.org/10 .1016/j.jgar.2019.10.028.

216. Lapuebla A, Abdallah M, Olafisoye O, Cortes C, Urban C, Landman D, Quale J. 2015. Activity of imipenem with relebactam against Gramnegative pathogens from New York City. Antimicrob Agents Chemother 59:5029-5031. https://doi.org/10.1128/AAC.00830-15.

217. Kaushik A, Ammerman NC, Lee J, Martins O, Kreiswirth BN, Lamichhane G, Parrish NM, Nuermberger EL. 2019. In vitro activity of the new $\beta$-lactamase inhibitors relebactam and vaborbactam in combination with $\beta$-lactams against Mycobacterium abscessus complex clinical Isolates. Antimicrob Agents Chemother 63:e02623-18. https://doi.org/10 .1128/AAC.02623-18.

218. Papp-Wallace KM, Barnes MD, Alsop J, Taracila MA, Bethel CR, Becka SA, van Duin D, Kreiswirth BN, Kaye KS, Bonomo RA. 2018. Relebactam is a potent inhibitor of the KPC-2 $\beta$-lactamase and restores imipenem susceptibility in KPC-producing Enterobacteriaceae. Antimicrob Agents Chemother 62:e00147-18. https://doi.org/10.1128/AAC.00174-18.

219. Carpenter J, Neidig N, Campbell A, Thornsberry T, Truex T, Fortney T, Zhang Y, Bush K. 2019. Activity of imipenem/relebactam against carbapenemase-producing Enterobacteriaceae with high colistin resistance. J Antimicrob Chemother 74:3260-3263. https://doi.org/10.1093/ jac/dkz354.

220. Fraile-Ribot PA, Zamorano L, Orellana R, del Barrio-Tofiño E, SánchezDiener I, Cortes-Lara S, López-Causapé C, Cabot G, Bou G, MartínezMartínez L, Oliver A, GEMARA-SEIMC/REIPI Pseudomonas study Group. 2019. Activity of imipenem/relebactam against a large collection of Pseudomonas aeruginosa clinical isolates and isogenic $\beta$-lactam resistant mutants. Antimicrob Agents Chemother 64:e02165-19. https://doi .org/10.1128/AAC.02165-19.

221. Noel AR, Bowker KE, Attwood M, MacGowan AP. 2019. Antibacterial effect of imipenem/relebactam on aerobic Gram-negative bacilli: in vitro simulations of 7 or 14 day human exposures. J Antimicrob Chemother 74:1945-1951. https://doi.org/10.1093/jac/dkz114.

222. Merck \& Co., Inc. 2019. RECARBRIO (package insert). Merck \& Co., Inc. Kenilworth, NJ.

223. Wu J, Racine F, Wismer MK, Young K, Carr DM, Xiao JC, Katwaru R, Si Q, Harradine P, Motyl M, Bhagunde PR, Rizk ML. 2018. Exploring the pharmacokinetic/pharmacodynamic relationship of relebactam (MK7655) in combination with imipenem in a hollow-fiber infection model. Antimicrob Agents Chemother 62:e02323-17. https://doi.org/10.1128/ AAC.02323-17.

224. European Medicines Agency. 2020. Recarbrio (imipenem/cilastatin/ relebactam) - an overview of Recarbrio and why it is authorised in the EU. European Medicines Agency, Amsterdam, Netherlands.

225. Sims M, Mariyanovski V, McLeroth P, Akers W, Lee Y-C, Brown ML, Du J, Pedley A, Kartsonis NA, Paschke A. 2017. Prospective, randomized, double-blind, phase 2 dose-ranging study comparing efficacy and safety of imipenem/cilastatin plus relebactam with imipenem/cilastatin 
alone in patients with complicated urinary tract infections. J Antimicrob Chemother 72:2616-2626. https://doi.org/10.1093/jac/dkx139.

226. Lucasti C, Vasile L, Sandesc D, Venskutonis D, McLeroth $P$, Lala $M$, Rizk ML, Brown ML, Losada MC, Pedley A, Kartsonis NA, Paschke A. 2016. Phase 2, dose-ranging study of relebactam with imipenemcilastatin in subjects with complicated intra-abdominal infection. Antimicrob Agents Chemother 60:6234-6243. https://doi.org/10 .1128/AAC.00633-16.

227. Motsch J, Murta de Oliveira C, Stus V, Köksal I, Lyulko O, Boucher HW, Kaye KS, File TM, Brown ML, Khan I, Du J, Joeng H-K, Tipping RW, Aggrey A, Young K, Kartsonis NA, Butterton JR, Paschke A. 2020. RESTORE-IMI 1: a multicenter, randomized, double-blind trial comparing efficacy and safety of imipenem/relebactam vs colistin plus imipenem in patients with imipenem-nonsusceptible bacterial infections. Clin Infect Dis 70:1799-1808. https://doi.org/10.1093/cid/ciz530.

228. ClinicalTrials.gov. 2019. Safety, tolerability, efficacy and pharmacokinetics of imipenem/cilastatin/relebactam (MK-7655A) in pediatric participants with Gram-negative bacterial infection (mk-7655a-021). https:// clinicaltrials.gov/ct2/show/NCT03969901.

229. ClinicalTrials.gov. 2017. Efficacy and safety of imipenem+cilastatin/ relebactam (MK-7655A) in Japanese participants with complicated intra-abdominal infection or complicated urinary tract infection (MK7655A-017). https://clinicaltrials.gov/ct2/show/NCT03293485.

230. ClinicalTrials.gov. 2018. Imipenem/cilastatin/relebactam (MK-7655A) versus piperacillin/tazobactam in participants with hospital-acquired or ventilator-associated bacterial pneumonia (MK-7655A-016). https:// clinicaltrials.gov/ct2/show/NCT03583333.

231. ClinicalTrials.gov. 2015. Imipenem/relebactam/cilastatin versus piperacillin/ tazobactam for treatment of participants with bacterial pneumonia (MK-7655A-014) (RESTORE-IMI 2). https://clinicaltrials.gov/ct2/show/ NCT02493764.

232. Livermore DM, Mushtaq S, Warner M, Turner SJ, Woodford N. 2018. Potential of high-dose cefepime/tazobactam against multiresistant Gramnegative pathogens. J Antimicrob Chemother 73:126-133. https://doi.org/ $10.1093 / \mathrm{jac} / \mathrm{dkx} 360$.

233. Sader HS, Castanheira M, Mendes RE, Flamm RK, Jones RN. 2017. Antimicrobial activity of high-proportion cefepime-tazobactam (WCK 4282) against a large number of Gram-negative isolates collected worldwide in 2014. Antimicrob Agents Chemother 61:e02409-16. https://doi.org/10.1128/AAC.02409-16.

234. Bansal N, Sukhwani KS, Kumar D S, Nambi PS, Gopalakrishnan R, Ramasubramanian V. 2018. Clinical efficacy and safety of cefepimetazobactam in hospitalized patients in South India. Infect Dis (Lond) 50:391-394. https://doi.org/10.1080/23744235.2017.1410284.

235. Ghafur A, Tayade A, Kannaian P. 2012. Clinical profile of patients treated with cefepime/tazobactam: a new $\beta$-lactam/ $\beta$-lactamase inhibitor combination. J Microbiol Infect Dis 2:79-86. https://doi.org/10 .5799/ahinjs.02.2012.03.0049.

236. ClinicalTrials.gov. 2018. Study of cefepime-tazobactam (FEP-TAZ) in complicated urinary tract infection (cUTI) or acute pyelonephritis (AP). https://clinicaltrials.gov/ct2/show/NCT03630081.

237. Papp-Wallace KM, Bethel CR, Caillon J, Barnes MD, Potel G, Bajaksouzian S, Rutter JD, Reghal A, Shapiro S, Taracila MA, Jacobs MR, Bonomo RA, Jacqueline C. 2019. Beyond piperacillin-tazobactam: cefepime and AAl101 as a potent $\beta$-lactam- $\beta$-lactamase inhibitor combination. Antimicrob Agents Chemother 63:e00105-19. https://doi.org/10.1128/AAC .00105-19.

238. Belley A, Huband MD, Fedler KA, Watters AA, Flamm RK, Shapiro S, Knechtle P. 2019. Development of broth microdilution mic and disk diffusion antimicrobial susceptibility test quality control ranges for the combination of cefepime and the novel $\beta$-lactamase inhibitor enmetazobactam. J Clin Microbiol 57:e00607-19. https://doi.org/10.1128/JCM .00607-19.

239. Morrissey I, Magnet S, Hawser S, Shapiro S, Knechtle P. 2019. In vitro activity of cefepime-enmetazobactam against Gram-negative isolates collected from U.S. and European hospitals during 2014-2015. Antimicrob Agents Chemother 63:e00514-19. https://doi.org/10 .1128/AAC.00514-19.

240. ClinicalTrials.gov. 2018. Safety and efficacy study of cefepimeAAI101 in the treatment of complicated urinary tract infections. https://clinicaltrials.gov/ct2/show/NCT03687255.

241. Papp-Wallace KM, Nguyen NQ, Jacobs MR, Bethel CR, Barnes MD, Kumar V, Bajaksouzian S, Rudin SD, Rather PN, Bhavsar S, Ravikumar T, Deshpande PK, Patil V, Yeole R, Bhagwat SS, Patel MV, van den Akker
F, Bonomo RA. 2018. Strategic approaches to overcome resistance against Gram-negative pathogens using $\beta$-lactamase inhibitors and $\beta$-lactam enhancers: activity of three novel diazabicyclooctanes WCK 5153, zidebactam (WCK 5107), and WCK 4234. J Med Chem 61: 4067-4086. https://doi.org/10.1021/acs.jmedchem.8b00091.

242. Avery LM, Mullane EM, Nicolau DP. 2020. Evaluation of the in vitro activity of WCK 5222 (cefepime/zidebactam) and currently available combination therapies against single- and double-carbapenemase producing Enterobacteriaceae: expanding the zone of hope. Int J Antimicrob Agents 55:105863. https://doi.org/10.1016/j.ijantimicag.2019.105863.

243. Sader HS, Rhomberg PR, Flamm RK, Jones RN, Castanheira M. 2017. WCK 5222 (cefepime/zidebactam) antimicrobial activity tested against Gramnegative organisms producing clinically relevant $\beta$-lactamases. J Antimicrob Chemother 72:1696-1703. https://doi.org/10.1093/jac/dkx050.

244. Khan Z, Iregui A, Landman D, Quale J. 2019. Activity of cefepime/ zidebactam (WCK 5222) against Enterobacteriaceae, Pseudomonas aeruginosa and Acinetobacter baumannii endemic to New York City medical centres. J Antimicrob Chemother 74:2938-2942. https://doi .org/10.1093/jac/dkz294.

245. Thomson KS, AbdelGhani S, Snyder JW, Thomson GK. 2019. Activity of cefepime-zidebactam against multidrug-resistant (MDR) Gramnegative pathogens. Antibiotics (Basel) 8:32. https://doi.org/10.3390/ antibiotics8010032.

246. Kaushik A, Ammerman NC, Parrish NM, Nuermberger EL. 2019. New $\beta$-lactamase inhibitors nacubactam and zidebactam improve the in vitro activity of $\beta$-lactam antibiotics against Mycobacterium abscessus complex clinical isolates. Antimicrob Agents Chemother 63:e00733-19. https://doi.org/10.1128/AAC.00733-19.

247. Kazmierczak KM, Bradford PA, Stone GG, de Jonge BLM, Sahm DF. 2018. In vitro activity of ceftazidime-avibactam and aztreonam-avibactam against OXA-48-carrying Enterobacteriaceae isolated as part of the International Network for Optimal Resistance Monitoring (INFORM) global surveillance program from 2012 to 2015. Antimicrob Agents Chemother 62:e00592-18. https://doi.org/10.1128/AAC.00592-18.

248. Sader HS, Mendes RE, Pfaller MA, Shortridge D, Flamm RK, Castanheira M. 2017. Antimicrobial activities of aztreonam-avibactam and comparator agents against contemporary (2016) clinical Enterobacteriaceae isolates. Antimicrob Agents Chemother 62:e01856-17. https://doi.org/ 10.1128/AAC.01856-17.

249. Karlowsky JA, Kazmierczak KM, de Jonge BLM, Hackel MA, Sahm DF, Bradford PA. 2017. In vitro activity of aztreonam-avibactam against Enterobacteriaceae and Pseudomonas aeruginosa isolated by clinical laboratories in 40 countries from 2012 to 2015. Antimicrob Agents Chemother 61:e00472-17. https://doi.org/10.1128/AAC.00472-17.

250. Biedenbach DJ, Kazmierczak K, Bouchillon SK, Sahm DF, Bradford PA 2015. In vitro activity of aztreonam-avibactam against a global collection of Gram-negative pathogens from 2012 and 2013. Antimicrob Agents Chemother 59:4239-4248. https://doi.org/10.1128/ AAC.00206-15.

251. ClinicalTrials.gov. 2017. A study to determine the efficacy, safety and tolerability of aztreonam-avibactam (ATM-AVI) \pm metronidazole (MTZ) versus meropenem (MER) \pm colistin (COL) for the treatment of serious infections due to Gram negative bacteria. (REVISIT). https://clinicaltrials .gov/ct2/show/NCT03329092.

252. Cornely OA, Cisneros JM, Torre-Cisneros J, Rodríguez-Hernández MJ Tallón-Aguilar L, Calbo E, Horcajada JP, Queckenberg C, Zettelmeyer U, Arenz D, Rosso-Fernández CM, Jiménez-Jorge S, Turner G, Raber S, O'Brien S, Luckey A, COMBACTE-CARE Consortium/REJUVENATE study Group. 2020. Pharmacokinetics and safety of aztreonam/avibactam for the treatment of complicated intra-abdominal infections in hospitalized adults: results from the REJUVENATE study. J Antimicrob Chemother 75:618-627. https://doi.org/10.1093/jac/dkz497.

253. Periasamy H, Joshi P, Palwe S, Shrivastava R, Bhagwat S, Patel M. 2020. High prevalence of Escherichia coli clinical isolates in India harbouring four amino acid inserts in PBP3 adversely impacting activity of aztreonam/avibactam. J Antimicrob Chemother 75:1650-1651. https:// doi.org/10.1093/jac/dkaa021.

254. Mojica MF, Papp-Wallace KM, Taracila MA, Barnes MD, Rutter JD, Jacobs MR, LiPuma JJ, Walsh TJ, Vila AJ, Bonomo RA. 2017. Avibactam restores the susceptibility of clinical isolates of Stenotrophomonas maltophilia to aztreonam. Antimicrob Agents Chemother 61:e00777-17. https://doi .org/10.1128/AAC.00777-17.

255. Durand-Réville TF, Guler S, Comita-Prevoir J, Chen B, Bifulco N, Huynh H, Lahiri S, Shapiro AB, McLeod SM, Carter NM, Moussa SH, Velez-Vega 
C, Olivier NB, McLaughlin R, Gao N, Thresher J, Palmer T, Andrews B, Giacobbe RA, Newman JV, Ehmann DE, de Jonge B, O'Donnell J, Mueller JP, Tommasi RA, Miller AA. 2017. ETX2514 is a broad-spectrum $\beta$-lactamase inhibitor for the treatment of drug-resistant Gramnegative bacteria including Acinetobacter baumannii. Nat Microbiol 2:17104. https://doi.org/10.1038/nmicrobiol.2017.104.

256. McLeod SM, Moussa SH, Hackel MA, Miller AA. 2020. In vitro activity of sulbactam-durlobactam against Acinetobacter baumanniicalcoaceticus complex isolates collected globally in 2016 and 2017. Antimicrob Agents Chemother 64:e02534-19. https://doi.org/10 .1128/AAC.02534-19.

257. Sagan O, Yakubsevitch R, Yanev K, Fomkin R, Stone E, Hines $D$, O'Donnell J, Miller A, Isaacs R, Srinivasan S. 2019. Pharmacokinetics and tolerability of intravenous sulbactam-durlobactam with imipenemcilastatin in hospitalized adults with complicated urinary tract infections, including acute pyelonephritis. Antimicrob Agents Chemother 64:e01506-19. https://doi.org/10.1128/AAC.01506-19.

258. ClinicalTrials.gov. 2019. Study to evaluate the efficacy and safety of intravenous sulbactam-ETX2514 in the treatment of patients with infections caused by Acinetobacter baumannii-calcoaceticus complex (ATTACK). https://clinicaltrials.gov/ct2/show/NCT03894046.

259. Mushtaq S, Vickers A, Woodford N, Haldimann A, Livermore DM. 2019. Activity of nacubactam (RG6080/OP0595) combinations against MBLproducing Enterobacteriaceae. J Antimicrob Chemother 74:953-960. https://doi.org/10.1093/jac/dky522.

260. Asempa TE, Motos A, Abdelraouf K, Bissantz C, Zampaloni C, Nicolau DP. 2020. Meropenem-nacubactam activity against AmpC-overproducing and KPC-expressing Pseudomonas aeruginosa in a neutropenic murine lung infection model. Int J Antimicrob Agents 55:105838. https://doi.org/10.1016/j.ijantimicag.2019.10.019.

261. Asempa TE, Motos A, Abdelraouf K, Bissantz C, Zampaloni C, Nicolau DP. 2019. Efficacy of human-simulated epithelial lining fluid exposure of meropenem-nacubactam combination against class A serine $\beta$-lactamase-producing Enterobacteriaceae in the neutropenic murine lung infection model. Antimicrob Agents Chemother 63:e02382-18. https://doi.org/10.1128/AAC.02382-18.

262. Monogue ML, Giovagnoli S, Bissantz C, Zampaloni C, Nicolau DP. 2018. In vivo efficacy of meropenem with a novel non- $\beta$-lactam- $\beta$-lactamase inhibitor, nacubactam, against Gram-negative organisms exhibiting various resistance mechanisms in a murine complicated urinary tract infection model. Antimicrob Agents Chemother 62:e02596-17. https:// doi.org/10.1128/AAC.02596-17.

263. Barnes MD, Taracila MA, Good CE, Bajaksouzian $S$, Rojas $L J$, van Duin $D$, Kreiswirth BN, Jacobs MR, Haldimann A, Papp-Wallace KM, Bonomo RA. 2019. Nacubactam enhances meropenem activity against carbapenemresistant Klebsiella pneumoniae producing KPC. Antimicrob Agents Chemother 63:e00432-19. https://doi.org/10.1128/AAC.00432-19.

264. Okujava R, Garcia-Alcalde F, Haldimann A, Zampaloni C, Morrissey I, Magnet S, Kothari N, Harding I, Bradley K. 2018. 1359. Activity of meropenem/nacubactam combination against Gram-negative clinical isolates: ROSCO global surveillance 2017. Open Forum Infect Dis 5:S416. https://doi.org/10.1093/ofid/ofy210.1190.

265. McLeod S, Carter N, Hackel M, Badal R, Mueller J, Tommasi R, Miller A. 2018. The novel $\beta$-lactamase inhibitor ETX1317 effectively restores the activity of cefpodoxime against extended spectrum $\beta$-lactamase (ESBL)- and carbapenemase-expressing Enterobacteriaceae isolated from recent urinary tract infections, poster 603 . Microbe 2018, Atlanta, GA.

266. Mcleod S, Moussa S, Huband M, Mueller J, Tommasi R, Miller A. 2019. The novel beta-lactamase inhibitor ETX1317 effectively restores the activity of cefpodoxime against recent global Enterobacteriaceae isolates from urinary tract infections, poster P1184. ECCMID 2019, Amsterdam, Netherlands.

267. Shapiro A, Moussa S, Carter N, Gao N, Miller AA. 2019. CefpodoximeETX1317 susceptibility is unaffected by ceftazidime-avibactam resistance mutations V240G, D179Y and D179Y/T243M in KPC-3 $\beta$-lactamase. Microbe 2019, San Francisco, CA.

268. Hamrick JC, Docquier J-D, Uehara T, Myers CL, Six DA, Chatwin CL, John KJ, Vernacchio SF, Cusick SM, Trout REL, Pozzi C, De Luca F, Benvenuti M, Mangani S, Liu B, Jackson RW, Moeck G, Xerri L, Burns CJ, Pevear DC, Daigle DM. 2019. VNRX-5133 (taniborbactam), a broad-spectrum inhibitor of serine- and metallo- $\beta$-lactamases, restores activity of cefepime in Enterobacterales and Pseudomonas aeruginosa. Antimicrob Agents Chemother 64:e01963-19. https://doi.org/10.1128/AAC.01963-19.
269. Daigle D, Hamrick J, Chatwin C, Kurepina N, Kreiswirth BN, Shields RK, Oliver A, Clancy CJ, Nguyen M-H, Pevear D, Xerri L. 2018. 1370. Cefepime/VNRX-5133 broad-spectrum activity is maintained against emerging KPC- and PDC-variants in multidrug-resistant $K$. pneumoniae and $P$ aeruginosa. Open Forum Infect Dis 5:S419-S420. https://doi.org/ 10.1093/ofid/ofy210.1201.

270. Hackel MA, Sahm D. 2018. In vitro activity of cefepime in combination with VNRX-5133 against meropenem and/or cefepime resistant clinical isolates of Pseudomonas aeruginosa, abstr 1544. ECCMID 2019, Madrid, Spain.

271. ClinicalTrials.gov. 2019. Safety and efficacy study of cefepime/VNRX5133 in patients with complicated urinary tract infections. https:// clinicaltrials.gov/ct2/show/NCT03840148.

272. Lomovskaya O, Lindley J, Rubio-Aparicio D, Nelson KJ, Castanheira M. 2019. 681. In vitro activity of the $\beta$-lactamase inhibitor QPX7728 in combination with several $\beta$-lactams against Acinetobacter baumannii (AB) and Pseudomonas aeruginosa (PSA). Open Forum Infect Dis 6 (Suppl 2):S310-S311. https://doi.org/10.1093/ofid/ofz360.749.

273. Castanheira M, Lindley J, Huynh H, Mendes RE, Lomovskaya O. 2019. 677. Activity of novel $\beta$-lactamase inhibitor QPX7728 combined with $\beta$-lactam agents when tested against carbapenem-resistant Enterobacteriaceae (CRE) isolates. Open Forum Infect Dis 6 (Suppl 2):S309-S309. https://doi.org/10.1093/ofid/ofz360.745.

274. Lomovskaya O, Nelson KJ, Rubio-Aparicio D. 2019. 727. Potency of the $\beta$-lactamase inhibitor QPX7728 is minimally affected by KPC mutations that reduce potency of ceftazidime-avibactam. Open Forum Infect Dis 6 (Suppl 2):S326-S326. https://doi.org/10.1093/ofid/ofz360.795.

275. Lomovskaya O, Nelson K, Rubio-Aparicio D, Tsivkovski R, Sun D, Dudley MN. 2020. Impact of intrinsic resistance mechanisms on potency of QPX7728, a new ultrabroad-spectrum beta-lactamase inhibitor of serine and metallo-beta-lactamases in Enterobacteriaceae, Pseudomonas aeruginosa, and Acinetobacter baumannii. Antimicrob Agents Chemother 64:e00552-20. https://doi.org/10.1128/AAC.00552-20.

276. Sader HS, Flamm RK, Carvalhaes CG, Castanheira M. 2020. Comparison of ceftazidime-avibactam and ceftolozane-tazobactam in vitro activities when tested against Gram-negative bacteria isolated from patients hospitalized with pneumonia in United States medical centers (2017-2018). Diagn Microbiol Infect Dis 96:114833. https://doi.org/10 .1016/j.diagmicrobio.2019.05.005.

277. Stone GG, Smayevsky J, Kazmierczak K. 2020. Longitudinal analysis of the in vitro activity of ceftazidime-avibactam vs. Pseudomonas aeruginosa, 2012-2016. Diagn Microbiol Infect Dis 96:114835. https://doi.org/ 10.1016/j.diagmicrobio.2019.05.007.

278. Ekkelenkamp MB, Cantón R, Díez-Aguilar M, Tunney MM, Gilpin DF, Bernardini F, Dale GE, Elborn JS, Bayjanov JR, Fluit A. 2019. Susceptibility of Pseudomonas aeruginosa recovered from cystic fibrosis patients to murepavadin and 13 comparator antibiotics. Antimicrob Agents Chemother 64:e01541-19. https://doi.org/10.1128/AAC.01541-19.

279. García-Fernández S, García-Castillo M, Bou G, Calvo J, Cercenado E, Delgado M, Pitart C, Mulet X, Tormo N, Mendoza DL, Díaz-Regañón J, Cantón R, SUPERIOR study group. 2019. Activity of ceftolozane/ tazobactam against Pseudomonas aeruginosa and Enterobacterales isolates recovered from intensive care unit patients in Spain: the SUPERIOR multicentre study. Int J Antimicrob Agents 53:682-688. https://doi.org/10.1016/j.jiantimicag.2019.02.004.

280. Gherardi G, Linardos G, Pompilio A, Fiscarelli E, Di Bonaventura G. 2019. Evaluation of in vitro activity of ceftolozane-tazobactam compared to other antimicrobial agents against Pseudomonas aeruginosa isolates from cystic fibrosis patients. Diagn Microbiol Infect Dis 94:297-303. https://doi.org/10.1016/j.diagmicrobio.2019.01.012.

281. Karlowsky JA, Kazmierczak KM, Bouchillon SK, de Jonge BLM, Stone GG, Sahm DF. 2019. In vitro activity of ceftazidime-avibactam against clinical isolates of Enterobacteriaceae and Pseudomonas aeruginosa collected in Latin American countries: results from the INFORM global surveillance program, 2012 to 2015. Antimicrob Agents Chemother 63:e01814-18. https://doi.org/10.1128/AAC.01814-18.

282. Liao C-H, Lee N-Y, Tang H-J, Lee SS-J, Lin C-F, Lu P-L, Wu J-J, Ko W-C, Lee W-S, Hsueh P-R. 2019. Antimicrobial activities of ceftazidime-avibactam, ceftolozane-tazobactam, and other agents against Escherichia coli, Klebsiella pneumoniae, and Pseudomonas aeruginosa isolated from intensive care units in Taiwan: results from the Surveillance of Multicenter Antimicrobial Resistance in Taiwan in 2016. Infect Drug Resist 12:545-552. https://doi.org/10.2147/IDR.S193638.

283. Sader HS, Castanheira M, Streit JM, Flamm RK. 2019. Frequency of 
occurrence and antimicrobial susceptibility of bacteria isolated from patients hospitalized with bloodstream infections in United States medical centers (2015-2017). Diagn Microbiol Infect Dis 95:114850. https://doi.org/10.1016/j.diagmicrobio.2019.06.002.

284. Karlowsky JA, Lob SH, Young K, Motyl MR, Sahm DF. 2018. Activity of imipenem/relebactam against Pseudomonas aeruginosa with antimicrobial-resistant phenotypes from seven global regions: SMART 2015-2016. J Glob Antimicrob Resist 15:140-147. https://doi.org/10 .1016/j.jgar.2018.07.012.

285. Karlowsky JA, Lob SH, Kazmierczak KM, Hawser SP, Magnet S, Young K, Motyl MR, Sahm DF. 2018. In vitro activity of imipenem/relebactam against Gram-negative ESKAPE pathogens isolated in 17 European countries: 2015 SMART surveillance programme. J Antimicrob Chemother 73:1872-1879. https://doi.org/10.1093/jac/dky107.

286. Shortridge D, Pfaller MA, Castanheira M, Flamm RK. 2018. Antimicrobial activity of ceftolozane-tazobactam tested against Enterobacteriaceae and Pseudomonas aeruginosa collected from patients with bloodstream infections isolated in United States hospitals (2013-2015) as part of the Program to Assess Ceftolozane-Tazobactam Susceptibility (PACTS) surveillance program. Diagn Microbiol Infect Dis 92:158-163. https://doi .org/10.1016/j.diagmicrobio.2018.05.011.

287. Sader HS, Flamm RK, Carvalhaes CG, Castanheira M. 2018. Antimicrobial susceptibility of Pseudomonas aeruginosa to ceftazidime-avibactam, ceftolozane-tazobactam, piperacillin-tazobactam, and meropenem stratified by U.S. census divisions: results from the 2017 INFORM program. Antimicrob Agents Chemother 62:e01587-18. https://doi.org/10 .1128/AAC.01587-18.

288. Henderson A, Tan E, McCarthy KL, Paterson DL. 2018. Activity of ceftolozane/tazobactam against a collection of Pseudomonas aeruginosa isolates from bloodstream infections in Australia. Pathology 50: 748-752. https://doi.org/10.1016/j.pathol.2018.08.009.

289. Zhou M, Chen J, Liu Y, Hu Y, Liu Y, Lu J, Zhang S, Yu Y, Huang X, Yang Q, Liao K, Jin Y, Huang W, Feng X, Yang Q, Xu Y. 2018. In vitro activities of ceftaroline/avibactam, ceftazidime/avibactam, and other comparators against pathogens from various complicated infections in China. Clin Infect Dis 67:S206-S216. https://doi.org/10.1093/cid/ciy659.

290. Kazmierczak KM, de Jonge BLM, Stone GG, Sahm DF. 2018. In vitro activity of ceftazidime/avibactam against isolates of Pseudomonas aeruginosa collected in European countries: INFORM global surveillance 2012-15. J Antimicrob Chemother 73:2777-2781. https://doi.org/ 10.1093/jac/dky267.

291. Sader HS, Huband MD, Duncan LR, Flamm RK. 2018. Ceftazidimeavibactam antimicrobial activity and spectrum when tested against Gram-negative organisms from pediatric patients: results from the INFORM surveillance program (United States, 2011-2015). Pediatr Infect Dis J 37:549-554. https://doi.org/10.1097/INF.0000000000001859.

292. Giani T, Arena F, Pollini S, Di Pilato V, D'Andrea MM, Henrici De Angelis L, Bassetti M, Rossolini GM, Vismara C, Luzzaro F, Cavallo R, Dusi PA, Pagani E, Sarti M, Farina C, Rigoli R, Scarparo C, Pecile P, Cusi MG, Mencacci A, Manso E, Spanu T, Labonia M, Tassi V, Amato G, Stefani S, Giraldi C, Rassu M, Pseudomonas aeruginosa Working Group. 2018. Italian nationwide survey on Pseudomonas aeruginosa from invasive infections: activity of ceftolozane/tazobactam and comparators, and molecular epidemiology of carbapenemase producers. J Antimicrob Chemother 73:664-671. https://doi.org/10.1093/jac/dkx453.

293. Shortridge D, Pfaller MA, Castanheira M, Flamm RK. 2018. Antimicrobial activity of ceftolozane-tazobactam tested against Enterobacteriaceae and Pseudomonas aeruginosa with various resistance patterns isolated in U.S. hospitals (2013-2016) as part of the surveillance program: program to assess ceftolozane-tazobactam susceptibility. Microb Drug Resist 24:563-577. https://doi.org/10.1089/mdr.2017.0266.

294. Lob SH, Hackel MA, Kazmierczak KM, Hoban DJ, Young K, Motyl MR, Karlowsky JA, Sahm DF. 2017. In vitro activity of imipenem-relebactam against Gram-negative bacilli isolated from patients with lower respiratory tract infections in the United States in 2015 - results from the SMART global surveillance program. Diagn Microbiol Infect Dis 88: 171-176. https://doi.org/10.1016/j.diagmicrobio.2017.02.018.

295. Pfaller MA, Bassetti M, Duncan LR, Castanheira M. 2017. Ceftolozane/ tazobactam activity against drug-resistant Enterobacteriaceae and Pseudomonas aeruginosa causing urinary tract and intraabdominal infections in Europe: report from an antimicrobial surveillance programme (2012-15). J Antimicrob Chemother 72:1386-1395. https://doi.org/10 .1093/jac/dkx009.

296. Pfaller MA, Shortridge D, Sader HS, Gales A, Castanheira M, Flamm RK.
2017. Ceftolozane-tazobactam activity against drug-resistant Enterobacteriaceae and Pseudomonas aeruginosa causing healthcareassociated infections in Latin America: report from an antimicrobial surveillance program (2013-2015). Braz J Infect Dis 21:627-637. https:// doi.org/10.1016/j.bjid.2017.06.008.

297. Pfaller MA, Shortridge D, Sader HS, Flamm RK, Castanheira M. 2017. Ceftolozane-tazobactam activity against drug-resistant Enterobacteriaceae and Pseudomonas aeruginosa causing healthcare-associated infections in Australia and New Zealand: report from an antimicrobial surveillance program (2013-2015). J Glob Antimicrob Resist 10: 186-194. https://doi.org/10.1016/j.jgar.2017.05.025.

298. Sader HS, Castanheira M, Flamm RK, Jones RN. 2016. Antimicrobial activities of ceftazidime-avibactam and comparator agents against Gram-negative organisms isolated from patients with urinary tract infections in U.S. medical centers, 2012 to 2014. Antimicrob Agents Chemother 60:4355-4360. https://doi.org/10.1128/AAC.00405-16.

299. Sader HS, Castanheira M, Flamm RK, Huband MD, Jones RN. 2016. Ceftazidime-avibactam activity against aerobic Gram-negative organisms isolated from intra-abdominal infections in United States hospitals, 2012-2014. Surg Infect (Larchmt) 17:473-478. https://doi.org/10 $.1089 /$ sur.2016.036.

300. Sader HS, Castanheira M, Farrell DJ, Flamm RK, Jones RN. 2015. Ceftazidime-avibactam activity when tested against ceftazidimenonsusceptible Citrobacter spp., Enterobacter spp., Serratia marcescens, and Pseudomonas aeruginosa from Unites States medical centers (2011-2014). Diagn Microbiol Infect Dis 83:389-394. https://doi.org/10 .1016/j.diagmicrobio.2015.06.008.

301. Sid Ahmed MA, Abdel Hadi H, Hassan AAl, Abu Jarir S, Al-Maslamani MA, Eltai NO, Dousa KM, Hujer AM, Sultan AA, Soderquist B, Bonomo RA, Ibrahim EB, Jass J, Omrani AS. 2019. Evaluation of in vitro activity of ceftazidime/avibactam and ceftolozane/tazobactam against MDR Pseudomonas aeruginosa isolates from Qatar. J Antimicrob Chemother 74: 3497-3504. https://doi.org/10.1093/jac/dkz379.

302. Karlowsky JA, Lob SH, Young K, Motyl MR, Sahm DF. 2019. Activity of imipenem-relebactam against multidrug-resistant Pseudomonas aeruginosa from the United States - SMART 2015-2017. Diagn Microbiol Infect Dis 95:212-215. https://doi.org/10.1016/j.diagmicrobio.2019.05 .001 .

303. Atkin SD, Abid S, Foster M, Bose M, Keller A, Hollaway R, Sader HS Greenberg DE, Finklea JD, Castanheira M, Jain R. 2018. Multidrugresistant Pseudomonas aeruginosa from sputum of patients with cystic fibrosis demonstrates a high rate of susceptibility to ceftazidimeavibactam. Infect Drug Resist 11:1499-1510. https://doi.org/10.2147/ IDR.S173804.

304. Schaumburg F, Bletz S, Mellmann A, Becker K, Idelevich EA. 2017. Susceptibility of MDR Pseudomonas aeruginosa to ceftolozane/ tazobactam and comparison of different susceptibility testing methods. J Antimicrob Chemother 72:3079-3084. https://doi.org/10.1093/ jac/dkx253.

305. Castanheira M, Duncan LR, Mendes RE, Sader HS, Shortridge D. 2017. Activity of ceftolozane-tazobactam against Pseudomonas aeruginosa and Enterobacteriaceae isolates collected from respiratory tract specimens of hospitalized patients in the United States during 2013 to 2015. Antimicrob Agents Chemother 62:e02125-17. https://doi.org/10.1128/ AAC.02125-17.

306. Giani T, Antonelli A, Sennati S, Di Pilato V, Chiarelli A, Cannatelli A, Gatsch C, Luzzaro F, Spanu T, Stefani S, Rossolini GM. 2020. Results of the Italian infection-Carbapenem Resistance Evaluation Surveillance Trial (iCREST-IT): activity of ceftazidime/avibactam against Enterobacterales isolated from urine. J Antimicrob Chemother 75:979-983. https:// doi.org/10.1093/jac/dkz547.

307. Zhou H, Zhang K, Chen W, Chen J, Zheng J, Liu C, Cheng L, Zhou W, Shen H, Cao X. 2020. Epidemiological characteristics of carbapenemresistant Enterobacteriaceae collected from 17 hospitals in Nanjing district of China. Antimicrob Resist Infect Control 9:15. https://doi.org/ 10.1186/s13756-019-0674-4.

308. Senchyna F, Gaur RL, Sandlund J, Truong C, Tremintin G, Kültz D, Gomez CA, Tamburini FB, Andermann T, Bhatt A, Tickler I, Watz N, Budvytiene I, Shi G, Tenover FC, Banaei N. 2019. Diversity of resistance mechanisms in carbapenem-resistant Enterobacteriaceae at a health care system in Northern California, from 2013 to 2016. Diagn Microbiol Infect Dis 93:250-257. https://doi.org/10.1016/j.diagmicrobio.2018.10 .004

309. Wilson WR, Kline EG, Jones CE, Morder KT, Mettus RT, Doi Y, Nguyen 
MH, Clancy CJ, Shields RK. 2019. Effects of KPC variant and porin genotype on the in vitro activity of meropenem-vaborbactam against carbapenem-resistant Enterobacteriaceae. Antimicrob Agents Chemother 63:e02048-18. https://doi.org/10.1128/AAC.02048-18.

310. Seifert $H$, Körber-Irrgang B, Kresken M, Göbel U, Swidsinski S, Rath P-M, Steinmann J, MacKenzie C, Mutters R, Peters G, Becker K, Podbielski A, Weise M, Siegel E, Glöckle B, Kniehl E, Becker A, Wichelhaus T-A, Schubert S, German ceftolozane/tazobactam study group. 2018. In-vitro activity of ceftolozane/tazobactam against Pseudomonas aeruginosa and Enterobacteriaceae isolates recovered from hospitalized patients in Germany. Int J Antimicrob Agents 51:227-234. https://doi.org/10.1016/ j.jjantimicag.2017.06.024.

311. Saran O, Sulik-Tyszka B, Basak GW, Wróblewska MM. 2019. Activity of ceftolozane/tazobactam against Gram-negative rods of the family Enterobacteriaceae and Pseudomonas spp. isolated from oncohematological patients hospitalized in a clinical hospital in Poland. Med Sci Monit 25:305-311. https://doi.org/10.12659/MSM.913352.

312. Hackel M, Kazmierczak KM, Hoban DJ, Biedenbach DJ, Bouchillon SK, de Jonge BLM, Stone GG. 2016. Assessment of the in vitro activity of ceftazidime-avibactam against multidrug-resistant Klebsiella spp. collected in the INFORM global surveillance study, 2012 to 2014. Antimicrob Agents Chemother 60:4677-4683. https://doi.org/10.1128/AAC .02841-15.

313. Vazquez JA, González Patzán LD, Stricklin D, Duttaroy DD, Kreidly Z, Lipka J, Sable C. 2012. Efficacy and safety of ceftazidime-avibactam versus imipenem-cilastatin in the treatment of complicated urinary tract infections, including acute pyelonephritis, in hospitalized adults: results of a prospective, investigator-blinded, randomized study. Curr Med Res Opin 28:1921-1931. https://doi.org/10.1185/03007995.2012 .748653 .

314. Lucasti C, Popescu I, Ramesh MK, Lipka J, Sable C. 2013. Comparative study of the efficacy and safety of ceftazidime/avibactam plus metronidazole versus meropenem in the treatment of complicated intraabdominal infections in hospitalized adults: results of a randomized, double-blind, phase II trial. J Antimicrob Chemother 68:1183-1192. https://doi.org/10.1093/jac/dks523.

315. Castón JJ, Lacort-Peralta I, Martín-Dávila P, Loeches B, Tabares S, Temkin L, Torre-Cisneros J, Paño-Pardo JR. 2017. Clinical efficacy of ceftazidime/avibactam versus other active agents for the treatment of bacteremia due to carbapenemase-producing Enterobacteriaceae in hematologic patients. Int J Infect Dis 59:118-123. https://doi.org/10 .1016/j.ijid.2017.03.021.

316. Temkin E, Torre-Cisneros J, Beovic B, Benito N, Giannella M, Gilarranz R, Jeremiah C, Loeches B, Machuca I, Jiménez-Martín MJ, Martínez JA, Mora-Rillo M, Navas E, Osthoff M, Pozo JC, Ramos Ramos JC, Rodriguez M, Sánchez-García M, Viale P, Wolff M, Carmeli Y. 2017. Ceftazidimeavibactam as salvage therapy for infections caused by carbapenemresistant organisms. Antimicrob Agents Chemother 61:e01964-16. https://doi.org/10.1128/AAC.01964-16.

317. Sousa A, Pérez-Rodríguez MT, Soto $A$, Rodríguez $L$, Pérez-Landeiro $A$ Martínez-Lamas L, Nodar A, Crespo M. 2018. Effectiveness of ceftazidime/avibactam as salvage therapy for treatment of infections due to OXA-48 carbapenemase-producing Enterobacteriaceae. J Antimicrob Chemother 73:3170-3175. https://doi.org/10.1093/jac/dky295.

318. Tumbarello M, Trecarichi EM, Corona A, De Rosa FG, Bassetti M, Mussini C, Menichetti F, Viscoli C, Campoli C, Venditti M, De Gasperi A, Mularoni A, Tascini C, Parruti G, Pallotto C, Sica S, Concia E, Cultrera R, De Pascale G, Capone A, Antinori S, Corcione S, Righi E, Losito AR, Digaetano M,
Amadori F, Giacobbe DR, Ceccarelli G, Mazza E, Raffaelli F, Spanu T, Cauda R, Viale P. 2019. Efficacy of ceftazidime-avibactam salvage therapy in patients with infections caused by Klebsiella pneumoniae carbapenemase-producing K. pneumoniae. Clin Infect Dis 68:355-364. https://doi.org/10.1093/cid/ciy492.

319. Miller B, Popejoy MW, Hershberger E, Steenbergen JN, Alverdy J. 2016. Characteristics and outcomes of complicated intra-abdominal infections involving Pseudomonas aeruginosa from a randomized, doubleblind, phase 3 ceftolozane-tazobactam study. Antimicrob Agents Chemother 60:4387-4390. https://doi.org/10.1128/AAC.03074-15.

320. Dinh A, Wyplosz B, Kernéis S, Lebeaux D, Bouchand F, Duran C, Béraud G, Lazaro P, Davido B, Hénard S, Canouï E, Ferry T, Wolff M. 2017. Use of ceftolozane/tazobactam as salvage therapy for infections due to extensively drug-resistant Pseudomonas aeruginosa. Int J Antimicrob Agents 49:782-783. https://doi.org/10.1016/j.ijantimicag.2017.04.001.

321. Munita JM, Aitken SL, Miller WR, Perez F, Rosa R, Shimose LA, Lichtenberger PN, Abbo LM, Jain R, Nigo M, Wanger A, Araos R, Tran TT, Adachi J, Rakita R, Shelburne S, Bonomo RA, Arias CA. 2017. Multicenter evaluation of ceftolozane/tazobactam for serious infections caused by carbapenem-resistant Pseudomonas aeruginosa. Clin Infect Dis 65: 158-161. https://doi.org/10.1093/cid/cix014.

322. Popejoy MW, Paterson DL, Cloutier D, Huntington JA, Miller B, Bliss CA, Steenbergen JN, Hershberger E, Umeh O, Kaye KS. 2017. Efficacy of ceftolozane/tazobactam against urinary tract and intra-abdominal infections caused by ESBL-producing Escherichia coli and Klebsiella pneumoniae: a pooled analysis of phase 3 clinical trials. J Antimicrob Chemother 72:268-272. https://doi.org/10.1093/jac/dkw374.

323. Tselepis L, Langley GW, Aboklaish AF, Widlake E, Jackson DE, Walsh TR, Schofield CJ, Brem J, Tyrrell JM. 2020. In vitro efficacy of imipenemrelebactam and cefepime-AAl101 against a global collection of ESBLpositive and carbapenemase-producing Enterobacteriaceae. Int J Antimicrob Agents 56:105925. https://doi.org/10.1016/j.ijantimicag.2020 .105925 .

324. Sader HS, Castanheira M, Huband M, Jones RN, Flamm RK. 2017. WCK 5222 (cefepime-zidebactam) antimicrobial activity against clinical isolates of Gram-negative bacteria collected worldwide in 2015. Antimicrob Agents Chemother 61:e00072-17. https://doi.org/10 .1128/AAC.00072-17.

325. Karaiskos I, Galani I, Souli M, Giamarellou H. 2019. Novel $\beta$-lactam$\beta$-lactamase inhibitor combinations: expectations for the treatment of carbapenem-resistant Gram-negative pathogens. Expert Opin Drug Metab Toxicol 15:133-149. https://doi.org/10.1080/17425255 .2019.1563071.

326. Theuretzbacher U, Bush K, Harbarth S, Paul M, Rex JH, Tacconelli E, Thwaites GE. 2020. Critical analysis of antibacterial agents in clinical development. Nat Rev Microbiol 18:286-298. https://doi.org/10.1038/ s41579-020-0340-0.

327. Papp-Wallace KM. 2019. The latest advances in $\beta$-lactam/ $\beta$-lactamase inhibitor combinations for the treatment of Gram-negative bacterial infections. Expert Opin Pharmacother 20:2169-2184. https://doi.org/10 .1080/14656566.2019.1660772.

328. Tamma PD, Hsu AJ. 2019. Defining the role of novel $\beta$-lactam agents that target carbapenem-resistant Gram-negative organisms. J Pediatric Infect Dis Soc 8:251-260. https://doi.org/10.1093/jpids/piz002.

329. van Duin D, Bonomo RA. 2016. Ceftazidime/avibactam and ceftolozane/ tazobactam: second-generation $\beta$-lactam/ $\beta$-lactamase inhibitor combinations. Clin Infect Dis 63:234-241. https://doi.org/10.1093/cid/ciw243. 
Dafna Yahav is an infectious diseases specialist at Rabin Medical Center, Beilinson Hospital, in Petah-Tikva, Israel, and a senior lecturer at Tel-Aviv University, Israel. She received her MD from Sackler Faculty of Medicine at Tel-Aviv University, Tel-Aviv, Israel. She completed her internal medicine residency in Beilinson Hospital, Petah-Tikva, Israel, and her infectious diseases fellowship as well. She has been working as an infectious disease specialist at Beilinson Hospital

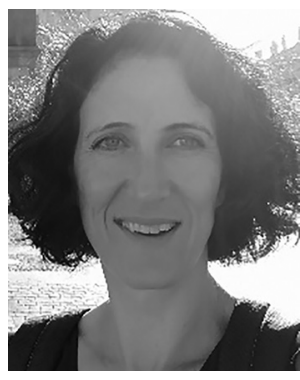
for the last 5 years. Her research interests focus on multidrug-resistant bacterial infections, infections in immunocompromised patients, and antibiotic treatment. She also has extensive experience in conducting systematic reviews and meta-analyses.

Christian G. Giske, MD/PhD, is a Professor at Karolinska Institutet, Stockholm, Sweden, and a Chief Consultant Physician in Clinical Bacteriology at Karolinska University Hospital, Stockholm, Sweden. He received his PhD degree from Karolinska Institutet and his MD degree from the Norwegian University for Science and Technology, Trondheim, Norway. He was trained as a clinical microbiologist at Karolinska University Hospital. Giske is the chair of the European Committee on

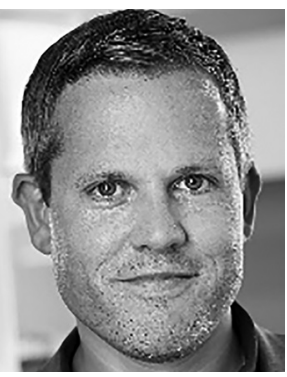
Antimicrobial Susceptibility Testing. His research interests include molecular epidemiology and bioinformatics, carriage of multidrug-resistant (MDR) bacteria, microbiome research, and antimicrobial susceptibility of novel antimicrobial agents. Dr. Giske has published more than 170 peer-reviewed articles listed on PubMed.

Alise Grāmatniece, MD, is an Internal Medicine Specialist, trainee in Infectious Diseases. She is a country representative for the Trainee Association of ESCMID, working as a researcher and lecturer at the University of Latvia and as a Medical Doctor at the Pauls Stradins Clinical University Hospital in Riga, Latvia. She has conducted her specialty training in Austria, the United States of America, and Sweden. Currently, she is a part of the COVID-19 response

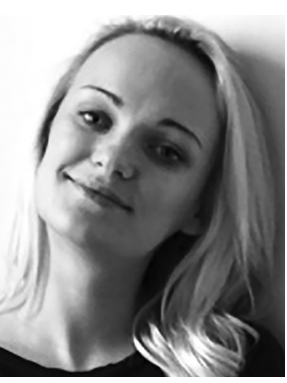
team. Her research interests include surveillance of nosocomial infections and outbreak investigation, antimicrobial susceptibility, antimicrobial stewardship, microbiome research, and molecular epidemiology.
Henrietta Abodakpi, PharmD, PhD, obtained both her PharmD. and PhD. degrees at the University of Houston College of Pharmacy. Under the mentorship of Dr. Vincent Tam, her graduate research focused on using pharmacokinetics/pharmacodynamics (PK/PD) to in form optimal dosing strategies for $\beta$-lactam$\beta$-lactamase inhibitor combinations in the treatment of infections caused by extendedspectrum $\beta$-lactamase-producing Enterobacteriaceae. She is currently a Clinical Pharmacol-

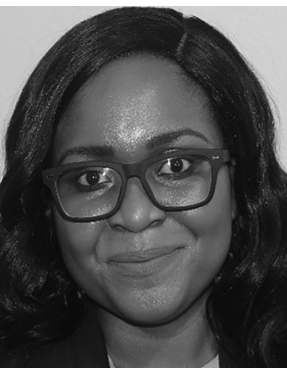
ogy Reviewer within the Division of Infectious Disease Pharmacology at the US Food and Drug Administration's Office of Clinical Pharmacology.

Vincent H. Tam, PharmD. is a Professor at the University of Houston College of Pharmacy in Houston, TX. He received a BS from the National University of Singapore, Singapore, and his PharmD from Albany College of Pharmacy in Albany, NY. He completed an infectious diseases pharmacy residency at Detroit Receiving Hospital in Detroit, Ml, and a clinical pharmacology/infectious diseases fellowship at Albany Medical College. He is board certified in pharmacotherapy with

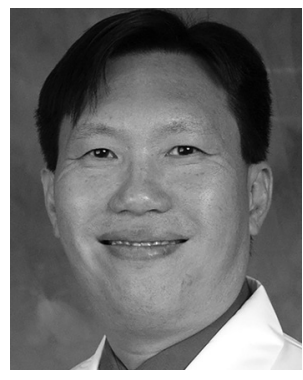
added qualifications in infectious diseases. His research interests have focused on antimicrobial pharmacokinetics/pharmacodynamics and infectious disease therapeutics. Dr. Tam has published over 160 peerreviewed articles in these areas.

Leonard Leibovici is a Professor of Medicine, Sackler Faculty of Medicine, Tel-Aviv University, and heads the Department of Medicine E, Beilinson Hospital, Rabin Medical Center, Petah-Tikva, Israel. He serves as the Editor-in-Chief of Clinical Microbiology and Infection. He studied medicine at Tel-Aviv University. His main research interests are antibiotic treatment, clinical studies, and evidence-based medicine. He has more than 350 publications on similar topics.

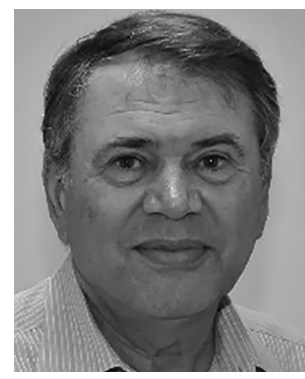

